Aus der Klinik für Hals-Nasen-Ohrenheilkunde

(Prof. Dr. med. D. Beutner)

der Medizinischen Fakultät der Universität Göttingen

\title{
Systematische radiographische Untersuchung von drei potentiellen Implantationslokalisationen für ein teilimplantiertes Knochenleitungshörsystem nach Mastoidchirurgie
}

\author{
INAUGURAL-DISSERTATION \\ zur Erlangung des Doktorgrades \\ für Zahnheilkunde \\ der Medizinischen Fakultät der \\ Georg-August-Universität zu Göttingen
}

vorgelegt von

Rebecka Scheele

aus

Vechta

Göttingen 2018 
Dekan:

\section{Betreuungsausschuss}

Betreuer:

Ko-Betreuer:

\section{Prüfungskommission}

Referent/in:

Ko-Referent/in:

Drittreferent/in:
Prof. Dr. rer. nat. H. K. Kroemer

Prof. Dr. med. F. Ihler

Priv.-Doz. Dr. med. J. M. Sohns

Prof. Dr. Friedrich Johannes Ihler

PD Dr. Daniel Behme

Prof. Dr. Rainer Mausberg

Datum der mündlichen Prüfung: 20. Mai 2019 
Hiermit erkläre ich, die Dissertation mit dem Titel „Systematische radiographische Untersuchung von drei potentiellen Implantationslokalisationen für ein teilimplantiertes Knochenleitungshörsystem nach Mastoidchirurgie" eigenständig angefertigt und keine anderen als die von mir angegebenen Quellen und Hilfsmittel verwendet zu haben.

Göttingen, den 


\section{Inhaltsverzeichnis}

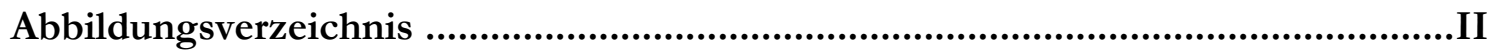

Tabellenverzeichnis ......................................................................................... III

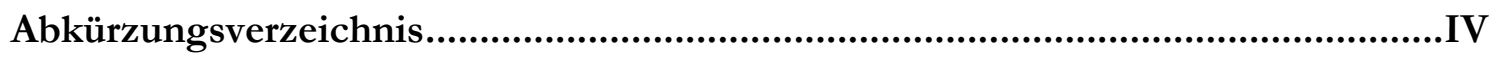

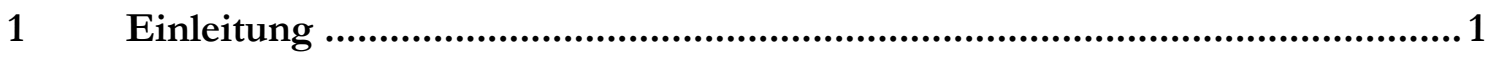

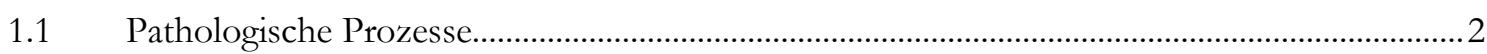

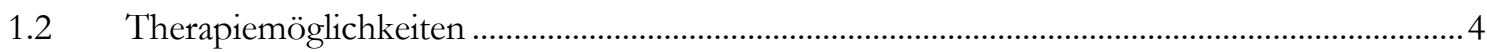

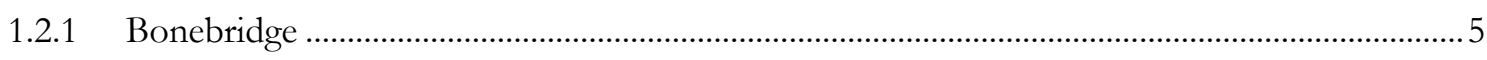

$1.3 \quad$ Fragestellung und Zielsetzung..........................................................................................

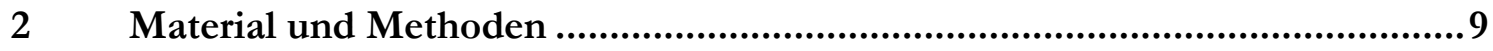

$2.1 \quad$ Ethische Unbedenklichkeit ...............................................................................................

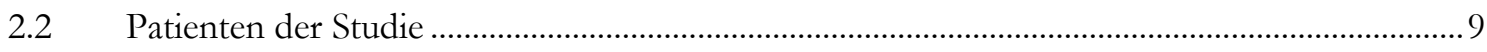

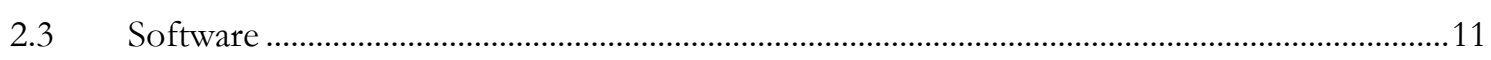

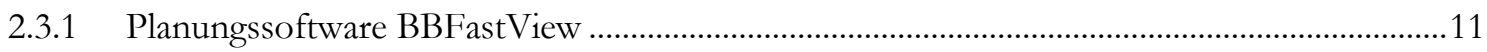

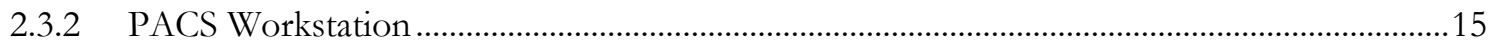

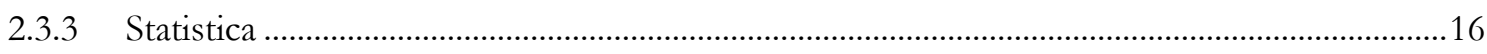

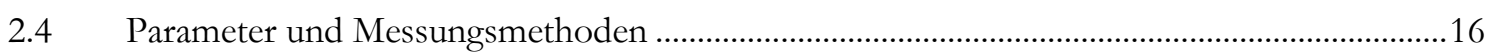

2.4.1 Beurteilungskriterien für retrosigmoidale und parietale Lokalisation ........................................18

2.4.2 Beurteilungskriterien für die Implantation im Mastoidresiduum ...........................................29

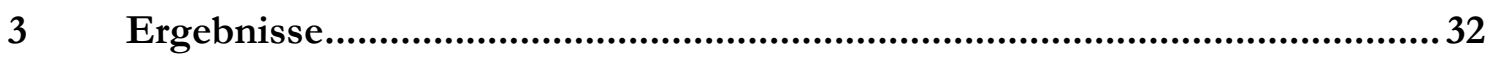

3.1 Virtuelle Implantation in der retrosigmoidalen Lokalisation ...................................................32

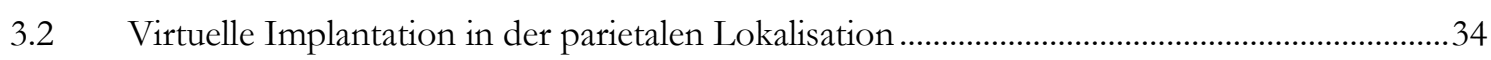

3.3 Virtuelle Implantation im Bereich des Mastoidresiduums........................................................35

$3.4 \quad$ Vergleich der drei Implantationslokalisationen ..........................................................................

$3.5 \quad$ Geschlechtsspezifische Unterschiede.........................................................................................

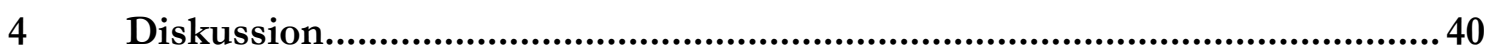

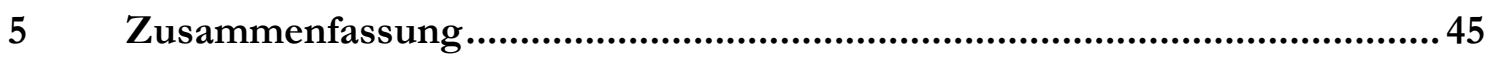

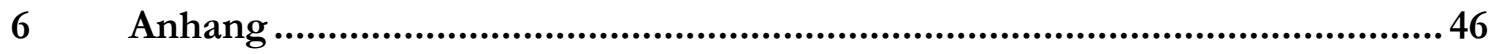

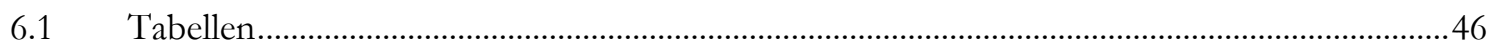

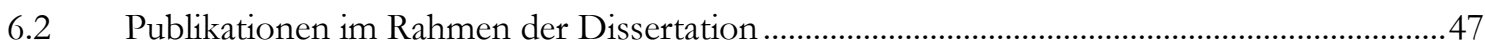

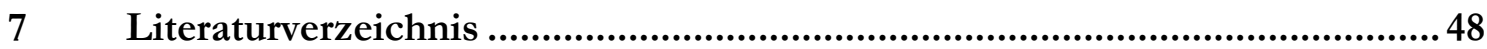




\section{Abbildungsverzeichnis}

Abbildung 1: Anatomie des Ohres. 2

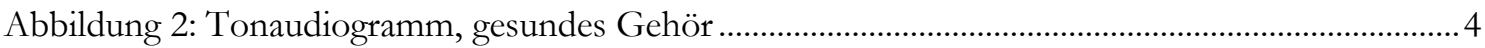

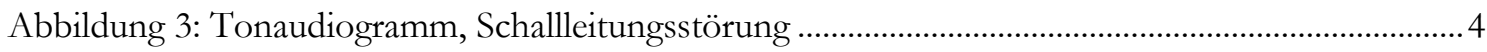

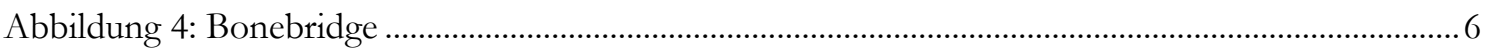

Abbildung 5: Teilimplantiertes Knochenleitungssystem mit extern getragenem Audioprozessor ......7

Abbildung 6: Übersicht der BBFastView-Fenster.........................................................................11

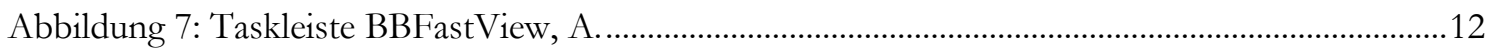

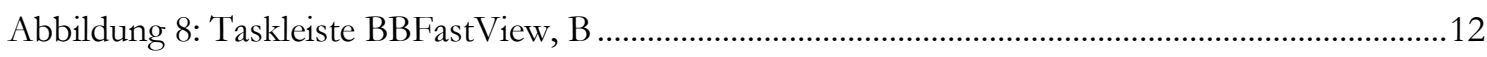

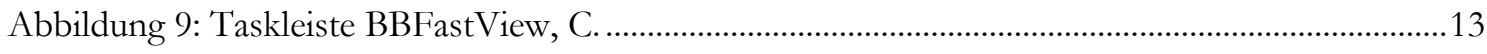

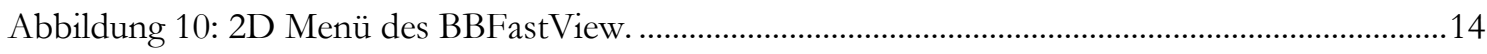

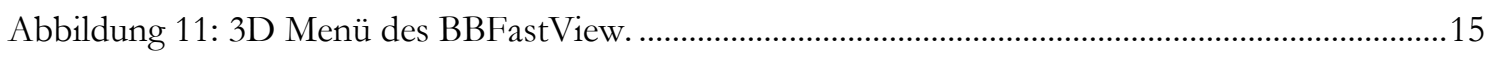

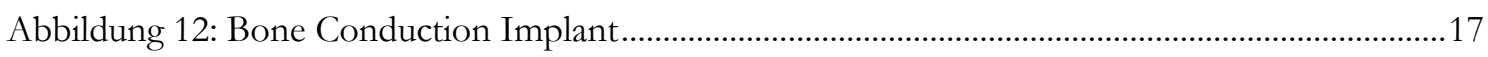

Abbildung 13: Dicke der Schädelkalotte mittig über dem BC-FMT....................................................18

Abbildung 14: Dicke der Schädelkalotte bei Schraubenöse 1 ............................................................19

Abbildung 15: Dicke der Schädelkalotte bei Schraubenöse 2 ...........................................................20

Abbildung 16: Maximale Duraimpression......................................................................................21

Abbildung 17: 3D-Visualisierung in BBFastView, Fläche der freiliegenden Implantatbasis

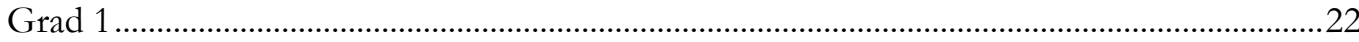

Abbildung 18: 3D-Visualisierung in BBFastView, Fläche der freiliegenden Implantatbasis Grad 2

Abbildung 19: 3D-Visualisierung in BBFastView, Fläche der freiliegenden Implantatbasis Grad 3

Abbildung 20: Minimale Entfernung des FMT-Zentrums zur Cochlea...............................................25

Abbildung 21: Winkel zwischen FMT-Zentrum und Frankfurter Horizontale ..................................26

Abbildung 22: Abknickungswinkel des FMT zur Spule ......................................................................27

Abbildung 23: Dicke der Kopfschwarte über dem Mittelpunkt der Spule .......................................28

Abbildung 24: Dicke der Kopfschwarte über dem Mittelpunkt des FMTs ......................................29

Abbildung 25: Kontakt zum Sinus sigmoideus .......................................................................................3

Abbildung 26: Maximale Dicke des nicht spongiösen Knochens bei Schraube 1..............................31

Abbildung 27: Virtuelle Implantation in der retrosigmoidalen Lokalisation .......................................332

Abbildung 28: Virtuelle Implantation in der parietalen Lokalisation.................................................34

Abbildung 29: Virtuelle Implantation in das Mastoidresiduum .............................................................36 


\section{Tabellenverzeichnis}

Tabelle 1: Messergebnisse und geschlechtsspezifische Unterschiede nach Implantation in die retrosigmoidale Lokalisation

Tabelle 2: Messergebnisse und geschlechtsspezifische Unterschiede nach Implantation in der parietalen Lokalisation

Tabelle 3: Messergebnisse und geschlechtsspezifische Unterschiede nach Implantation in das Mastoidresiduum

Tabelle 4: Vergleich der drei Implantationslokalisationen. ...................................................................47 


\section{Abkürzungsverzeichnis}

$\begin{array}{ll}\text { BAHA } & \text { Bone Anchored Hearing Aid } \\ \text { BC-FMT } & \text { Bone Conduction- Floating Mass Transducer } \\ \text { BCI } & \text { Bone Conduction Implant } \\ \text { CROS } & \text { Contralateral Routing Of Signals } \\ \text { CT } & \text { Computertomographie } \\ \text { DICOM } & \text { Digital Imaging and COmmunications in Medicine } \\ \text { PACS } & \text { Picture Archiving and Communication System } \\ \text { SA } & \text { Standardabweichung }\end{array}$




\section{$1 \quad$ Einleitung}

Das Hören, einer der komplexesten Vorgänge im menschlichen Körper, ist Voraussetzung für die Teilnahme am gesellschaftlichen Geschehen. Nach der Global Burden of DiseaseStudie der World Health Organization (WHO) gehört eine Hörstörung zu den Erkrankungen, welche die Lebensqualität der Betroffenen am meisten beeinflusst (Mathers et al. 2000). Laut der Studie von Sohn waren bereits 1999 19\% der deutschen Bevölkerung von einer behandlungsbedürftigen Hörstörung betroffen (Sohn 2001). Es wird bei den Hörstörungen zwischen Schallleitungsstörungen, Schallempfindungsstörungen, Schallverarbeitungsstörungen sowie Kombinationen aus diesen unterschieden. Diese Begriffe beziehen sich auf die Lokalisation der Störung im auditorischen System (Zahnert 2011).

Die Schallleitungsstörung beschreibt eine mechanische Unterbrechung in der Übertragung der Schallwellen von der Ohrmuschel über den äußeren Gehörgang auf das Trommelfell (Membrana tympani) sowie weiter über Hammer, Amboss und Steigbügel, die in der luftgefüllten Paukenhöhle (Cavitas tympani, Mittelohr) liegen. Vom Steigbügel werden die Schwingungen auf die Peri- und Endolymphe des Innenohres übertragen. Kommt es in diesem Verlauf zu Unterbrechungen, sind die Schallwellen zu schwach, um die Basilarmembran in den Cochleawindungen (Hörschnecke; Innenohr) in Schwingung zu versetzen. Als Folge findet keine Auslenkung der auf den Sinneszellen sitzenden Zilien statt und die Umwandlung von mechanischer Energie in elektrische Impulse bleibt aus. Schließlich leitet der Hörnerv keine Informationen zum auditorischen Cortex weiter (siehe Abbildung 1, (Schmidt 2017)). Der Patient ist taub oder zumindest schwerhörig. 


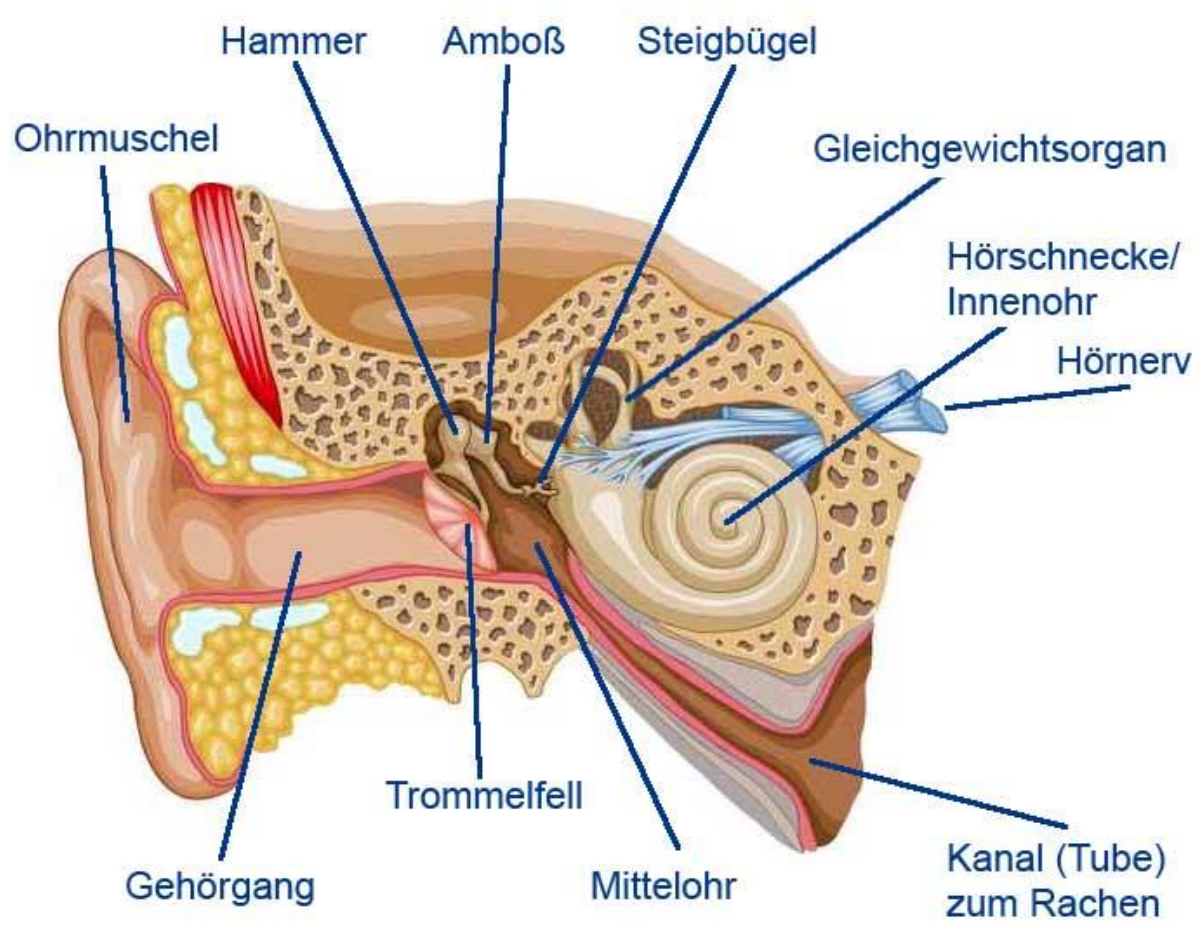

Abbildung 1: Anatomie des Ohres. Im koronaren Schnitt durch das Felsenbein sind die äußere Ohrmuschel mit Gehörgang sowie das Trommelfell zu sehen. Es schließt sich die Paukenhöhle (Mittelohr) mit der Gehörknöchelchenkette (Hammer, Amboss, Steigbügel) an. Über das ovale Fenster gelangen Schallwellen in das Innenohr (Cochlea und Labyrinth), bevor es hier zur Umwandlung in elektrische Reize kommt, die über den Nervus vestibulococblearis zum auditorischen Cortex geleitet werden. Illustration mit freundlicher Genehmigung von Werner Schmidt, www.werner-schmidt-wittenberg.de.

\subsection{Pathologische Prozesse}

Eine Mittelohrentzündung (Otitis media) stört die oben beschriebene Schallleitung. Es werden akute und chronische Formen der Otitis media unterschieden, mit denen vorübergehende oder permanente Schallleitungsstörungen einhergehen. Von der akuten Form sind insbesondere Kinder in den ersten drei Lebensjahren betroffen (Fiellau-Nikolajsen 1983). Die engen anatomischen Verhältnisse und die Infektanfälligkeit lassen Viren und Bakterien infolge banaler Infekte der oberen Atemwege über die Tuba auditiva ins Mittelohr aufsteigen. Die häufig in Zusammenhang stehende chronische Tubenventilationsstörung behindert die Pneumatisationsvorgänge während des Wachstums (Boenninghaus und Lenarz 2007). Eine heute durch antibiotische Therapiemöglichkeiten selten gewordene Komplikation der akuten Mittelohrentzündung ist die Entzündung des Warzenfortsatzes. Bei der Mastoiditis kommt es zur eitrigen Einschmelzung der unter physiologischen Bedingungen pneumatisierten Zellen des Felsenbeins. Dies kann sich je nach Ausprägungsgrad der Pneuma- 
tisation auch auf Zellen des Jochbogenansatzes sowie Zellen der Felsenbeinspitze ausweiten. In einer Computertomographie (CT) können die durch die Entzündung aufgelösten Knochensepten als weichteildichte Strukturen sichtbar werden. Die Therapie ist in diesem Akutstadium immer chirurgischer Natur. Um eine Drainage für das entzündliche Sekret zu schaffen, müssen die betroffenen Knochenareale radikal ausgebohrt werden (Boenninghaus und Lenarz 2007).

Die Otitis media chronica tritt vor allem bei Patienten auf, die im Kleinkindalter während der Pneumatisationsvorgänge an permanent unzureichender Tubenbelüftung litten (Boenninghaus und Lenarz 2007). Dies wird insbesondere bei Kindern mit Lippen-Kiefer-GaumenSpalten sowie Patienten mit Trisomie 21 beobachtet (Zahnert 2011), da Gaumen- und Tubenfehlbildungen u. a. Merkmale der Syndrome sind. Bei der chronisch verlaufenden Mittelohrentzündung wird eine chronische Schleimhauteiterung (chronische mesotympanale Otitis media) von einer chronischen Knocheneiterung (chronische epitympanale Otitis media) abgegrenzt. Beide Formen gehen mit Hörverlusten und Trommelfelldefekten einher (Gürkov und Nagel 2013). Eine chronische mesotympanale Otitis media wird von einem Umbauprozess der Schleimhaut im Mesotympanon begleitet und verläuft ohne Schäden des Knochengewebes. Das pathologische Epithel produziert ein schleimig-eitriges, aber geruchsloses Sekret, welches unter jeder Infektion durch die Tube oder den Gehöhrgang auftritt (Boenninghaus und Lenarz 2007). Ein weiteres Hauptsymptom ist die Schallleitungsschwerhörigkeit, die ebenso bei der chronischen epitympanalen Mittelohrentzündung vorliegt. Differentialdiagnostisch wird bei letzterer das Übergreifen der Entzündung auch auf knöcherne Strukturen beschrieben sowie eine fötide Eiterung aus dem Ohr. Dieses Symptom kann über Jahre bestehen (Boenninghaus und Lenarz 2007). Ursächlich für die chronisch epitympanale Otitis media sind langanhaltende Tubenventilationsstörungen, die zu einem Unterdruck in der Paukenhöhle führen, da kein physiologischer Druckausgleich über den Nasopharynx gewährleistet ist. Im Trommelfell können sich durch die pathologischen Druckverhältnisse so genannte Retraktionstaschen bilden (Gürkov und Nagel 2013). Hier kommt es zur Abschilferung des Epithels, das von einer Schicht aus verhornendem Plattenepithel und einer entzündlichen Perimatrix umgeben wird und auch als Perlgeschwulst oder Cholesteatom bezeichnet wird. Ein fortlaufender Entzündungsreiz führt zu einem Knochenabbau, der sich auf benachbarte Strukturen ausbreiten kann. Mögliche Komplikationen können deshalb mit Schwindel, Erbrechen oder Schallempfindungsschwerhörigkeit im Rahmen einer Innenohrbeteiligung (Labyrinthitis) sowie Benommenheit, Fieber, Schüttelfrost oder Fazialisparese einhergehen (Boenninghaus und Lenarz 2007). Die Therapie der Wahl ist wie auch bei der Mastoiditis operativ. Die von der Entzündung ergriffenen Knochenanteile müssen ausgebohrt werden und bei großer Ausdehnung wird eine nach außen offene Mastoidhöhle (sog. Radikalhöhle) angelegt. Zusätzlich sind nicht selten Tympanoplastiken zur Rekonstruktion des durch die Entzündung geschädigten Schallleitungsapparats notwendig (Boenninghaus und Lenarz 2007; Gürkov und Nagel 2013). 


\subsection{Therapiemöglichkeiten}

Schallleitungsstörungen liegen oft in einer Episode jahrelanger, entzündlicher Geschehnisse begründet. Eine häufig angewandte Therapie zur Verbesserung des Hörvermögens im Rahmen von Schallleitungshörschäden ist die Rekonstruktion des Trommelfells sowie der nachfolgenden Gehörknöchelchenkette im Rahmen einer Tympanoplastik. Führt dies zu keinem ausreichend gutem Hörvermögen, so müssen Hörsysteme zum Tragen kommen. Bei einigen Patienten sind konventionelle Hörgeräte, die insbesondere bei höhergradigen Schwerhörigkeiten mit einer Okklusion des Gehörganges einhergehen würden, nicht zielführend einzusetzen. Anatomische Besonderheiten des äußeren Ohres (Mikrotie/ Atresie) oder aber auf Grund von entzündlichen Prozessen bestehende chronische Otorrhoe sind nur einige Kontraindikationen (Edmiston et al. 2015). Bei der Schallleitungsschwerhörigkeit zeigt sich eine Differenz zwischen der Hörschwellenbestimmung über die Knochenleitung und der Luftleitung (Boenninghaus und Lenarz 2007). Siehe hierzu auch Abbildung 2 und Abbildung 3 (Boenninghaus und Lenarz 2007).

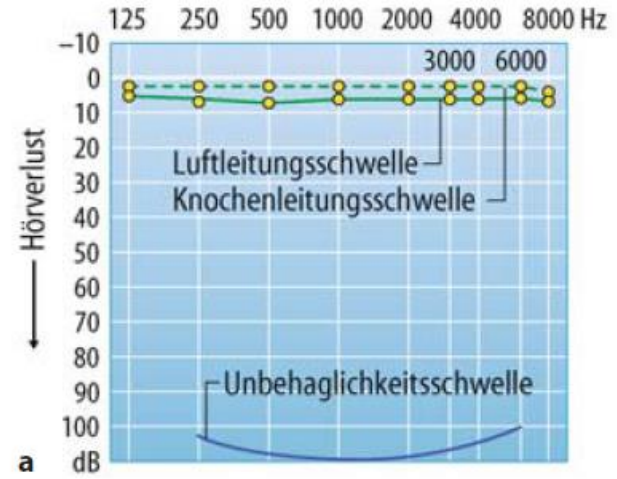

Abbildung 2: Tonaudiogramm, gesundes Gehör. Luftleitungsschwelle (durchgezogene Linie) und Knochenleitungsschwelle (gestrichelte Linie). Illustration mit freundlicher Genehmigung des Springer Verlags (modifiziert).

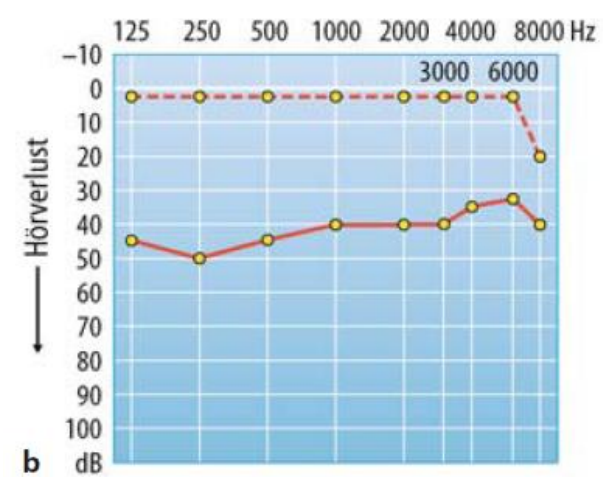

Abbildung 3: Tonaudiogramm, Schallleitungsstörung. Die Schwelle für Luftleitung (durchgezogene Linie) und die Knochenleitungsschwelle (gestrichelte Linie) liegen etwa $40 \mathrm{~dB}$ auseinander. Während die Hörschwelle für Luftleitung bei der Schallleitungsstörung deutlich erhöht ist, ist die Schallempfindung über die Knochenleitung unverändert. Illustration mit freundlicher Genehmigung des Springer Verlags (modifiziert).

Diese Knochenleitung, die beispielsweise im Test mit einer angestoßenen Stimmgabel überprüft wird und auch beim Klang der eigenen Stimme wahrgenommen wird, ist für Therapiezwecke anwendbar. Hierbei kann eine Störung im Schallleitungsapparat umgangen werden. Insbesondere bei Kleinkindern, wo ein früher operativer Eingriff vermieden werden soll und dennoch das Hören insbesondere für den Spracherwerb zentrale Bedeutung in 
der Entwicklung hat, können Bone Anchored Hearing Aid (BAHA)-Systeme angewandt werden, die beispielsweise mit Hilfe eines elastischen Stimbands auf den Schädelknochen gedrückt werden (Zarowski et al. 2011). Es handelt sich demnach um ein passives Knochenleitungssystem. Diese Systeme wandeln die Schallenergie in Vibrationen um, die über die Haut und den Schädelknochen, aber auch über Weichteile (Zahnert 2011) bis an das Innenohr weitergeführt werden. Dort kann es dann zur physiologischen Umwandlung in elektrische Reize kommen, die vom Hörnerv zur Hörrinde geleitet werden. Die BAHA kann zudem als perkutanes System über eine knochenverankerte Titanschraube genutzt werden. Diese wird über das Prinzip der Osseointegration, die von dem Schweden PerIngvar Brånemark geprägt wurde, in den Schädelknochen perkutan eingeschraubt (Albrektsson et al. 1981) und der Audioprozessor extern über beispielsweise ein Druckknopfsystem aufgesetzt. Die Aktivierung bzw. das Ansetzen des Audioprozessors darf jedoch erst nach abgeschlossener Osseointegration stattfinden, welches einige Wochen in Anspruch nimmt (Clemente et al. 2011). Mit dem Begriff Osseointegration wird die direkte Anlagerung von Knochen an die Fixierungsschrauben beschrieben. Durch physiologische Prozesse mit Hilfe von Osteoklasten und -blasten entsteht eine Einheit ohne fibröses Bindegewebe als Verbinder, sodass ein heller Klopfschall ein Indikator für eine gelungene Osseointegration ist. Dieses Prinzip wird heute routinemäßig in der dentalen Implantologie verwendet (Holtmann et al. 2016, S. 28). Der erste Patient wurde 1977 mit dem System versorgt (Tjellström und Granström 1994). Vorteile gegenüber der Stirnband-getragenen Variante sind hier die bessere Übertragungsqualität und Lautstärke insbesondere in hohen Frequenzbereichen (Zarowski et al. 2011). Weiter werden jedoch eine umfangreiche Pflege der perkutan liegenden Titanschraube als Nachteil genannt sowie das Auftreten von Hautirritationen und Entzündungen (Bosman et al. 2001). In einer Studie aus Manchester, United Kingdom (UK) wurden 602 implantierte BAHAs aus dem Zeitraum 1984 bis 2008 untersucht, und es konnte eine allgemeine Komplikationsrate von 23,9 \% festgestellt werden (Hobson et al. 2010). Hauptsächlich habe es sich dabei um Weichgewebswucherungen, Infektionen sowie Lockerungen und Verlust der Titanschraube gehandelt. Anhaltende Schmerzen, freiliegender Schädelknochen sowie Taubheitsgefühle der betroffenen Hautareale werden als seltene Komplikationen von Hobson et. al. beschrieben (Hobson et al. 2010).

\subsubsection{Bonebridge}

Das aktive Knochenleitungshörsystem Bonebridge (Fa. MED-EL, Innsbruck, Österreich) (siehe Abbildung 4) löst die für die perkutanen Systeme typischen Infektionsprobleme. Als teilimplantiertes Hörsystem ist der aktive Anteil des Gerätes unter der intakten Kopfschwarte gelegen und ein extern getragener Audioprozessor fängt die Schallwellen der Umgebung auf. Der Halt zwischen dem Audioprozessor und dem implantierten Anteil, dem BCI (Bone Conduction Implant) erfolgt magnetisch, sodass der externe Teil nach Belieben und insbesondere zur Nacht entfernt werden kann. Der zu implantierende Anteil des Knochen- 
leitungshörsystems besteht aus drei zusammenhängenden Komponenten: der Spule, dem Demodulator und dem Bone Conduction-Floating Mass Transducer (BC-FMT) (siehe Abbildung 5). Der Audioprozessor gibt die Schallwellen in Form elektrischer Signale über die intakte Kopfhaut an die Spule weiter. Der Demodulator setzt die Signale in mechanische Schwingungen um und überträgt diese auf das BC-FMT. Dieser Aktuator ist über zwei Schrauben in der Schädelkalotte fest verankert, sodass über diese die Vibrationen auf den Knochen übertragen werden können. Eine zeitintensive Osseointegration entfällt bei diesem System und die Aktivierung der Bonebridge kann bereits sieben Tage postoperativ stattfinden (Barbara et al. 2013). Durch Umgehung des Außen- und Mittelohres erfolgt die Weiterleitung direkt an das Innenohr, wodurch Peri- und Endolymphe der Cochlea in Schwingung versetzt werden können. Es folgt der physiologische Hörprozess mit Umwandlung der Schwingungen in elektrische Signale und Reizung des Nervus vestibulocochlearis. Dieses transkutane Knochenleitungshörsystem eignet sich wie auch die anderen Knochenleitungshörsytseme besonders zur Therapie von Patienten, die an einer Schallleitungsstörung sowie einer kombinierten Hörstörung oder einseitiger Taubheit erkrankt sind. Bei zuletzt genanntem erfolgt die Verbesserung der Hörsituation durch eine Übertragung der auf der ertaubten Seite aufgenommenen Schallwellen über die Knochenleitung an das gesunde Gegenohr (CROS'1-System).

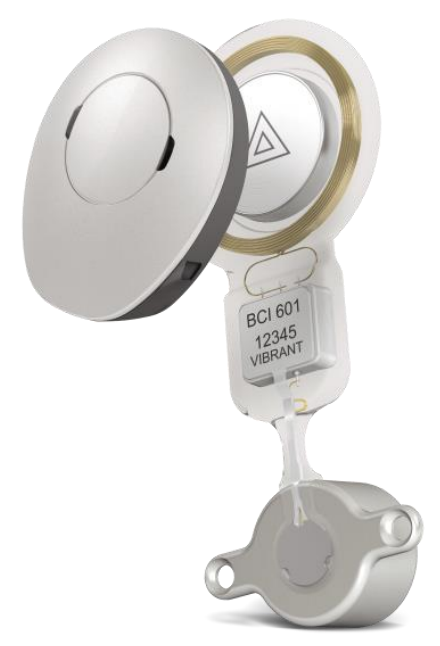

Abbildung 4: Bonebridge. Dieses aktive teilimplantierte Knochenleitungshörsystem besteht aus einem implantierten Teil (BCI) und einem externen Audioprozessor. Illustration mit freundlicher Genehmigung der Fa. MED-EL.

${ }^{1}$ CROS: Contralateral Routing Of Signals 
Der Vorteil gegenüber den herkömmlichen Hörgeräten ist die nicht erforderliche Okklusion des Gehörganges, welche beispielsweise bei aktiven Entzündungen zur Unterhaltung dieser beitragen kann. Auch erübrigen sich aufgrund der geschlossenen Kopfhaut des transkutanen Systems die bei perkutanen Geräten häufig erforderlichen zeitintensiven Pflegerituale der Titanschraube wie auch Kontrolluntersuchungen. Darüber hinaus wird der erhebliche kosmetische Vorteil, der zur besseren Akzeptanz beim Patienten führen kann in der Literatur beschrieben (Huber et al. 2013).

Zudem zeigt eine Studie von Håkansson et al., dass der audiologische Erfolg zwischen dem BCI (transkutan) und der BAHA (Classic-300, perkutan) vergleichbar bzw. im Hinblick auf das transkutane System geringfügig besser ist (Håkansson et al. 2008). Eine jüngere Studie an lediglich drei Patienten kann insbesondere bei Schallleitungsschwerhörigkeit eine Verbesserung im Sprachverständnis mit einer Erniedrigung der Hörschwelle von zuvor 37dB auf $14 \mathrm{~dB}$ nachweisen (Hassepass et al. 2015). In der Literatur wird jedoch als mögliches Problem die Größe des BC-FMTs der Bonebridge beschrieben (Håkansson et al. 2010; Barbara et al. 2013; Edmiston et al. 2015).

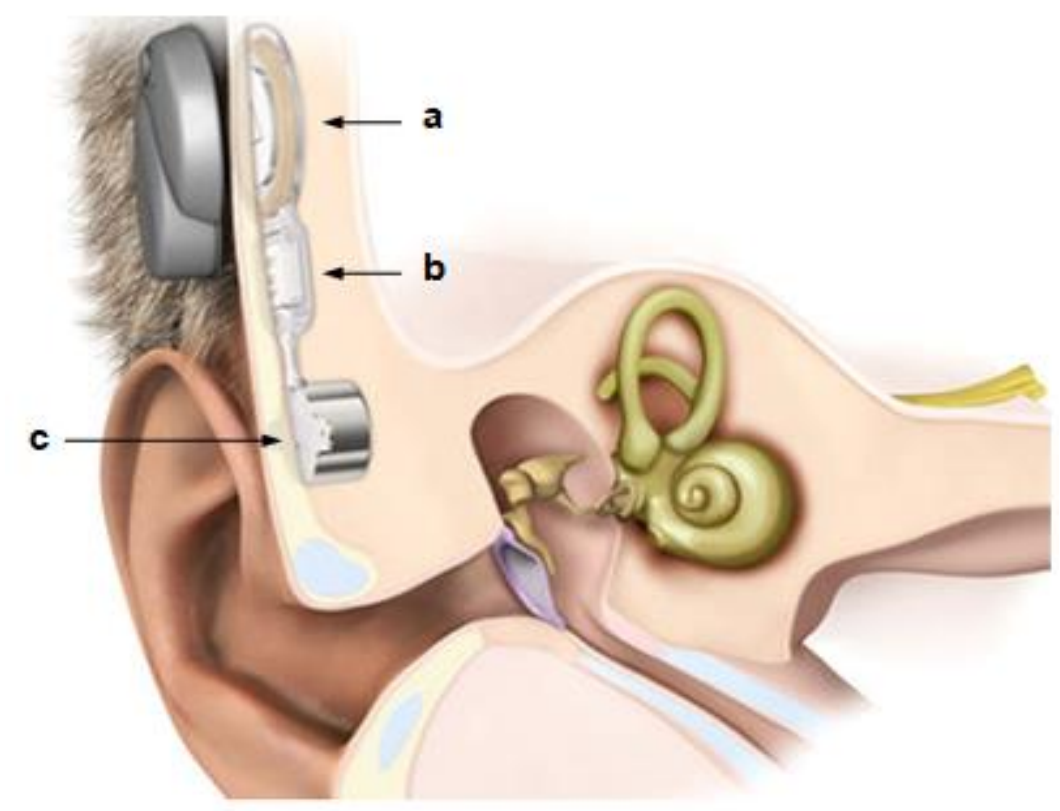

Abbildung 5: Teilimplantiertes Knochenleitungshörsystem mit extern getragenem Audioprozessor. Die Abbildung zeigt im koronaren Schnitt das Hörorgan. Kranial der Ohrmuschel ist im Deckhaar der extern getragene Audioprozessor gelegen. Unter der intakten Kopfschwarte liegt der zu implantierende Anteil der Bonebridge: a) Spule b) Demodulator c) BC-FMT. Illustration mit freundlicher Genehmigung der Firma MED-EL, Innsbruck, Österreich (modifiziert).

\subsection{Fragestellung und Zielsetzung}

Teilimplantierte aktive transkutane Knochenleitungshörsysteme eignen sich zur Therapie einer Schallleitungsschwerhörigkeit, kombinierten Hörstörung oder als CROS-System zur 
Verbesserung des Hörvermögens bei einseitiger Taubheit. Üblicherweise ist für die Implantation des zylinderförmigen Aktuators (Wandler, BC-FMT) der Bereich im Mastoid vorgesehen. Der Zylinder hat eine Tiefe von 8,7 mm und einen Durchmesser von 15,8 mm, sodass in die Schädeldecke eine entsprechende Kavität präpariert werden muss. Im Bereich des Warzenfortsatzes ist häufig ausreichend Knochensubstanz vorhanden und die Lokalisation ist nah am physiologischen Hörprozess gelegen. Der extern getragene Audioprozessor kann im Bereich der oberen Ohrmuschelkante gut verdeckt unter dem Kopfhaar getragen werden und ermöglicht bei entsprechendem Hörvermögen auf der Gegenseite ein Richtungshören, wie es physiologisch das äußere Ohr übernehmen würde. Erkrankungen wie das Cholesteatom gehen häufig mit chirurgischer Therapie und gleichzeitiger Schallleitungsstörung einher. Auch sind rezidivierende Entzündungen mit Otorrhoe häufig, sodass ein konventionelles Gerät wenig geeignet ist. Für diese Patienten ist die Bonebridge meist eine gute Versorgungsmöglichkeit, um das Gehör zu rehabilitieren. Die als Standard beschriebene Implantationsmöglichkeit im Bereich des Mastoids (MED-EL 2012; Canis et al. 2013) ist bei diesen Patienten, die als Folge chirurgischer Interventionen beträchtliche Anteile ihres knöchernen Warzenfortsatzes verloren haben, jedoch häufig nicht möglich. Folglich sind alternative Implantationslokalisationen für den verhältnismäßig großen Aktuator gefragt.

In der Literatur wird die retrosigmoidale Region als Alternative bereits beschrieben (Barbara et al. 2013; Canis et al. 2013; Wimmer et al. 2015). Weitere Alternativen könnten der Bereich dorsokranial der Sutura parietomastoidea sein (Zernotti und Sarasty 2015) sowie das Mastoid, welches individuell je nach vorheriger chirurgischer Intervention noch mehr oder weniger vorhanden ist. Im Rahmen dieser Arbeit wurde untersucht, inwieweit die drei Lokalisationen restrosigmoidal, parietal und das Mastoid nach vorausgegangener Operation als sinnvolle, mögliche Implantationsbereiche für den Aktuator der Bonebridge angesehen werden können. Um die einzelnen Positionen zu bewerten, wurde mit Hilfe einer Software namens BBFastView eine virtuelle Implantation an Schnittbildern kranialer Computertomographien durchgeführt. Die CT-Bilder entsprachen Patientenschädeln, die bereits chirurgische Interventionen im Bereich des Mastoids erfahren hatten. Nach bestmöglicher virtueller Implantation erfolgten Messungen, anhand derer die verschiedenen Lokalisationen beschrieben und verglichen wurden. Beispiele sind die Notwendigkeit der Duraimpression, falls die Dicke des Schädelknochens die 8,7 mm des BC-FMTs unterschreitet, oder aber auch die Distanz zur Cochlea, damit im Zuge der Schallleitung möglichst wenig Energie für die zu überwindende Strecke verloren geht (Eeg-Olofsson et al. 2008). Ziel war es, die Eignung unterschiedlicher Implantationslokalisationen für ein teilimplantiertes transkutanes aktives Knochenleitungshörsystem zu bewerten. 


\section{$2 \quad$ Material und Methoden}

Im Folgenden werden die Voraussetzungen für die Durchführung dieser Studie sowie die Methodik der Messungen im Detail beschrieben. Es wird sowohl auf die ethischen Aspekte der Patientenstudie als auch auf Ein- und Ausschlusskriterien eingegangen. Unter 2.3.1 wird die Software BBFastView vorgestellt, sodass die Nutzung und Handhabung deutlich wird. Die Bewertungskriterien der drei Lokalisationen werden in 2.4 ausführlich beschrieben und jeweils mit Bildmaterial aus dem praktischen Teil dieser Arbeit unterlegt.

\subsection{Ethische Unbedenklichkeit}

Im Rahmen der vorliegenden Arbeit wurden bereits vorhandene CTs des Schädels des Instituts für Diagnostische und Interventionelle Radiologie der Universitätsmedizin Göttingen genutzt. Demnach kommt es bei keinem Patienten der Studie zu einer zusätzlichen Strahlenbelastung oder anderen körperlichen Beeinträchtigungen, was ethisch fragwürdig wäre. Der Speicherung der Patientendaten wurde zuvor zugestimmt und demnach auch die Gewissheit gegeben, dass diese Daten vertraulich behandelt werden. Wie in jedem medizinischen Bereich liegen die Patientendaten auch im Rahmen dieser Arbeit selbstverständlich unter strenger Geheimhaltung vor. Der praktische Forschungsteil dieser Arbeit wurde innerhalb der Universitätsmedizin durchgeführt und im Rahmen des schriftlichen Aufgabenbereichs wurden lediglich anonymisierte Daten niedergeschrieben.

Für dieses Projekt erfolgte eine Beratung und Bewertung des Vorhabens durch die Ethikkommission der Universitätsmedizin Göttingen und ein entsprechender Antrag auf Prüfung der ethischen Unbedenklichkeit des Projektvorhabens wurde am 09. Dezember 2015 positiv bewertet (Antragsnummer DOK_346_2015). Davon gesondert erfolgte die Prüfung des Promotionsvorhabens mit positiver Bewertung am 17. März 2016 unter anderen durch den Vorsitzenden der Ethikkommission, Prof. Dr. Jürgen Brockmöller.

\subsection{Patienten der Studie}

Bei den im Rahmen der Studie untersuchten kranialen CTs handelte es sich um bereits vorliegende Aufnahmen, die mit Hilfe folgender Suchbegriffe bzw. Zeichenkette von Herrn Priv.-Doz. Dr. med. Jan M. Sohns (Institut für Diagnostische und Interventionelle Radiologie, Universitätsmedizin Göttingen) identifiziert wurden:

(Schädel-CT oder CT-Schädel oder CCT oder Kopf-CT oder CT-Kopf oder Cranielles CT oder Neurokranium oder Neurocranium oder Schädel CT oder CT Schädel); (Radikalhöhle oder Mastoidhöhle oder Ohrradikalhöhle oder Mastoidektomie) 
Insgesamt ergab diese Suche 135 Untersuchungen, die an einer PACS²-Workstation unter Berücksichtigung folgender Ein- und Ausschlusskriterien betrachtet wurden:

Einschlusskriterien:

- CT-Untersuchung in Feinschicht $(0,6 \mathrm{~mm})$

- chirurgische Intervention an mindestens einem Mastoid

Ausschlusskriterien:

- Patienten haben das 18. Lebensjahr noch nicht vollendet

- Patient trägt bereits ein Hörsystem in situ

- CT zeigt Artefakte, die die Auswertung unmöglich machen

- Patienten, die mehrmals vom System aufgerufen wurden, werden lediglich einmal in die Studie einbezogen; hier in der Regel das jüngste CT

Abschließend kam es bei 52 Patienten-CTs zum Einschluss in die Studie. 25 Patienten waren männlich und 27 Patienten weiblich. Da insgesamt acht Patienten vorausgegangene chirurgische Intervention im Bereich beider Mastoide aufwiesen, konnten insgesamt 60 Schädelhälften (Fälle) genutzt werden, um virtuell das BC-FMT zu implantieren und im Folgenden die Lokalisation als mögliche Implantationsalternative zu bewerten. Die Patienten waren zum Zeitpunkt der Bildgebung im Mittel 68,2 Jahre alt; die Altersspanne reichte von 22,2 bis zu 92,7 Jahren.

Bei den der CT-Bildgebung vorausgegangenen chirurgischen Eingriffen der Patienten handelte es sich in 32 Fällen um eine canal-wall-up-Mastoidektomie und in 28 Fällen um einen canal-wall-down-Eingriff. Bei beiden Operationen handelt es sich um Eingriffe am Felsenbein, wobei der Unterschied im Erhalt der Gehörgangswand liegt. Bei der canal-wall-upChirurgie zur Cholesteatomentfernung wird die Rezidivgefahr aufgrund der eingeschränkteren Sichtverhältnisse als höher eingestuft, der Gehörgang bleibt aber intakt. Bei der canalwall-down-Chirurgie wird die Gehörgangswand entfernt, was die Sicht verbessert, wobei jedoch eine Mastoidhöhle verbleibt (Ent USA 2017).

2 PACS: Picture Archiving and Communication System 


\subsection{Software}

\subsubsection{Planungssoftware BBFastView}

Die Planungssoftware BBFastView (CEIT und Tecnun Universidad de Navarra, DonostiaSan Sebastian, Spain; (Ceit 2017)) steht kostenfrei zur Verfügung und ermöglicht es, die DICOM ${ }^{3}-D a t e n$ der Patienten-CTs in drei Ebenen (sagittal, axial, koronar) zu betrachten. Zudem kann eine weitere Option namens 3D View hinzugeschaltet werden (siehe Abbildung 6), sodass über eine Rekonstruktion der 2D-Bilder der Schädel in 3D betrachtet werden kann. Über die Taskleiste Session können sowohl neue Patientenfälle geladen New from DICOM... als auch gespeicherte Daten geöffnet werden Open... (siehe Abbildung 7).

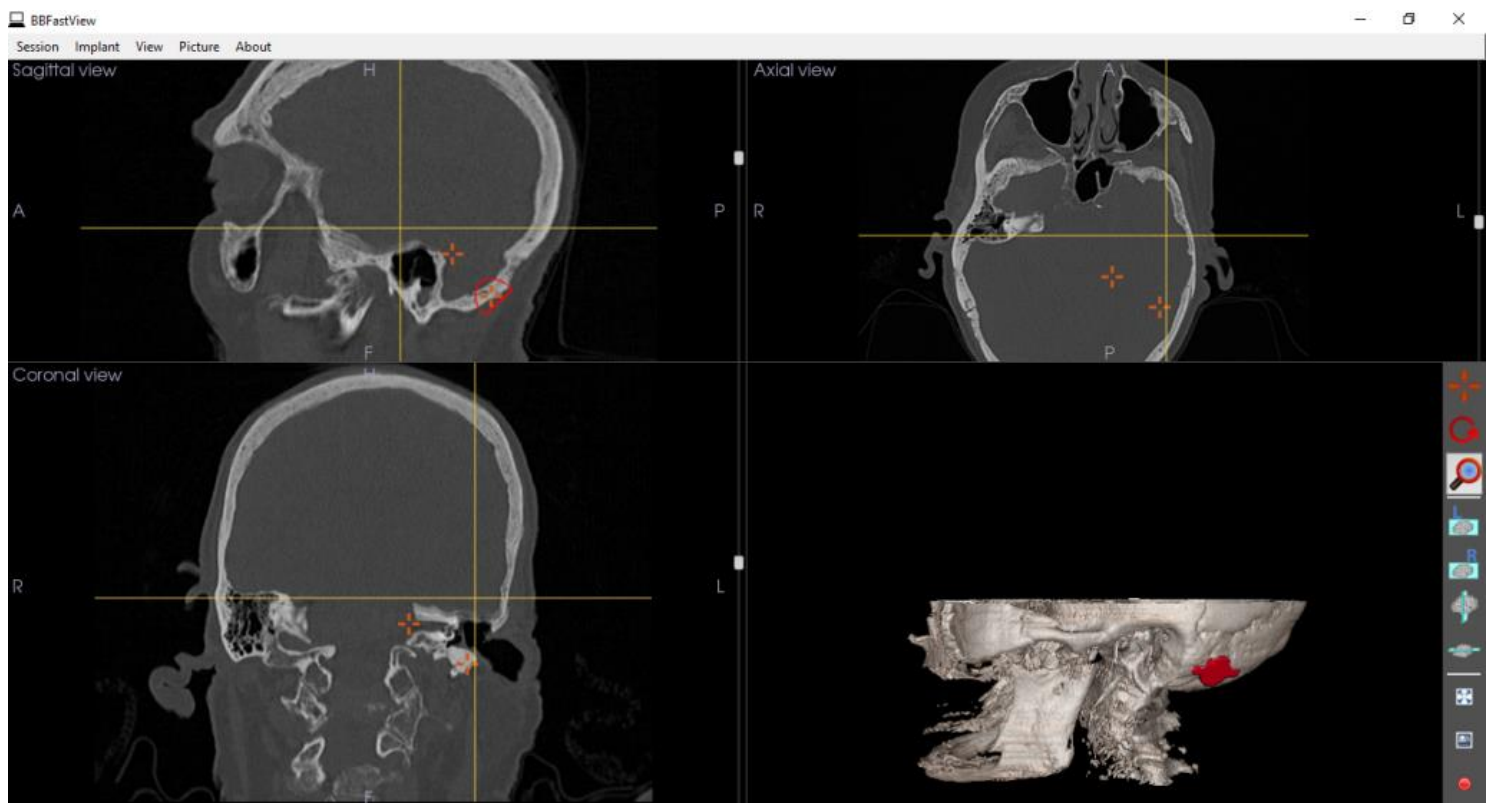

Abbildung 6: Übersicht der BBFastView-Fenster. Diese Bildschirmaufnahme zeigt die im BBFastView geladenen DICOM Daten. Oben links saggitale Ansicht, oben rechts axiale Ansicht, unten links koronare Ansicht, unten rechts 3D-Rekonstruktion (3D View). In roter Farbe ist das Template des Aktuators platziert. 


\section{BBFastView}

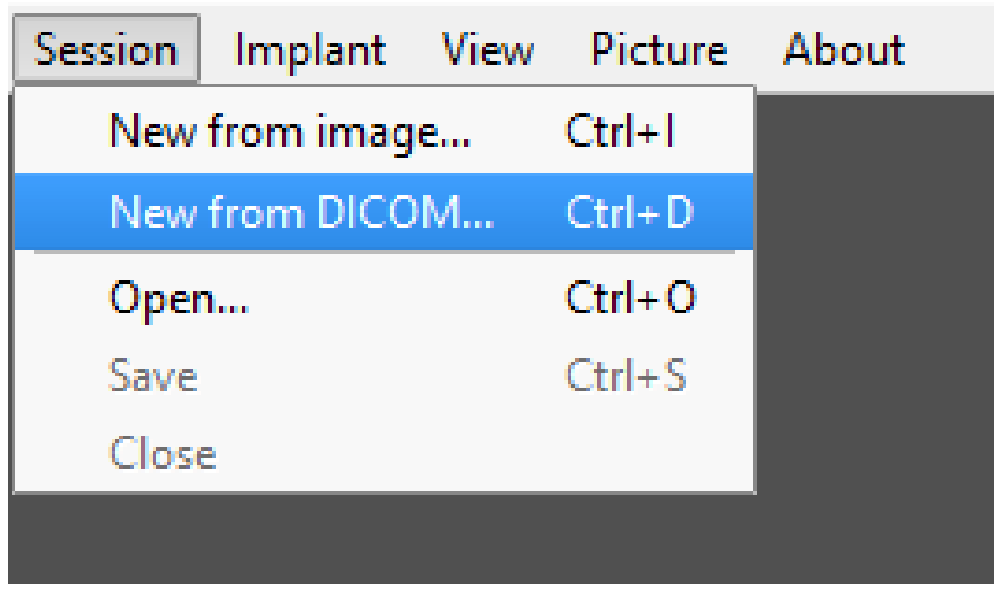

Abbildung 7: Taskleiste BBFastView, A. Mit Hilfe der Option New from DICOM... können neue Patientenfälle in die Software geladen werden. Über Open ... werden gespeicherte Fälle erneut aufgerufen.

Über Implant - Load BC-FMT_3D_Template_FINAL.vtk kann der Aktuator des teilimplantierten transkutanen Knochenleitungshörsystem als Schablone (template) geladen werden (siehe Abbildung 8). Die Schablone wurde von dem Hersteller der Bonebridge MED-EL GmbH, Innsbruck, Österreich bereitgestellt.

\section{BBFastView}

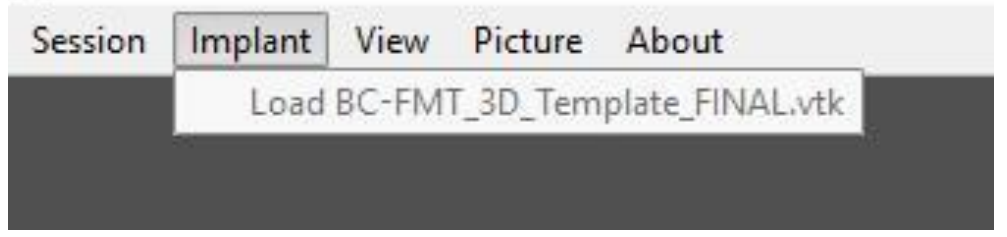

Abbildung 8: Taskleiste BBFastView, B. Über die Option Implant kann das von der Firma MED-EL bereitgestellte template geladen werden, sodass es im Kontext mit den CT-Bildern bearbeitet werden kann.

Sind CT-Bilder und template geladen, kann der Aktuator mit Hilfe seiner zwei Fadenkreuze per drag and drop intuitiv mit der Computermaus platziert werden. Es hat sich bewährt während der Platzierung und der anschließenden Messungen über die Taskleistenoption View die 3D-Visualisierung ausgeschaltet zu lassen. Zudem sollte beim Hinzufügen der 3DRekonstruktion unter dem Befehlsfeld View die GPU visualization und das Feld Annotations eingeschaltet sein (siehe Abbildung 9), um eine bessere Darstellung des Schädelknochens zu erhalten. 


\section{BBFastView}

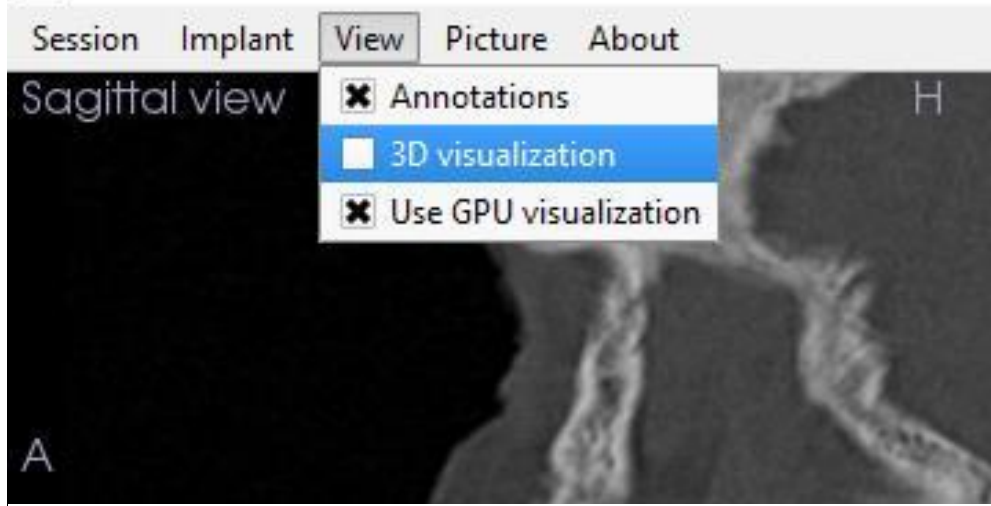

Abbildung 9: Taskleiste BBFastView, C. Unter View kann die Anzeige der 3D-Rekonstruktion hinzugewählt werden. Annotations und Use GPU visualization waren während der Erarbeitung dieses Projektes aktiviert.

Zur Durchführung der Messungen kann mit Hilfe der rechten Computermaustaste eine Menüleiste geöffnet werden (siehe Abbildung 10), durch die sich einfache Befehle durchführen lassen, wie beispielsweise Zoom, Pan und Fullscreen, um sich das Bild in Größe und Position individuell einstellen zu können. Weiter wurden über Befehle wie Distance und Angle die notwendigen Messungen durchgeführt. Die Option Implant muss immer aktiviert sein, wenn das template in seiner Position geändert werden soll. 


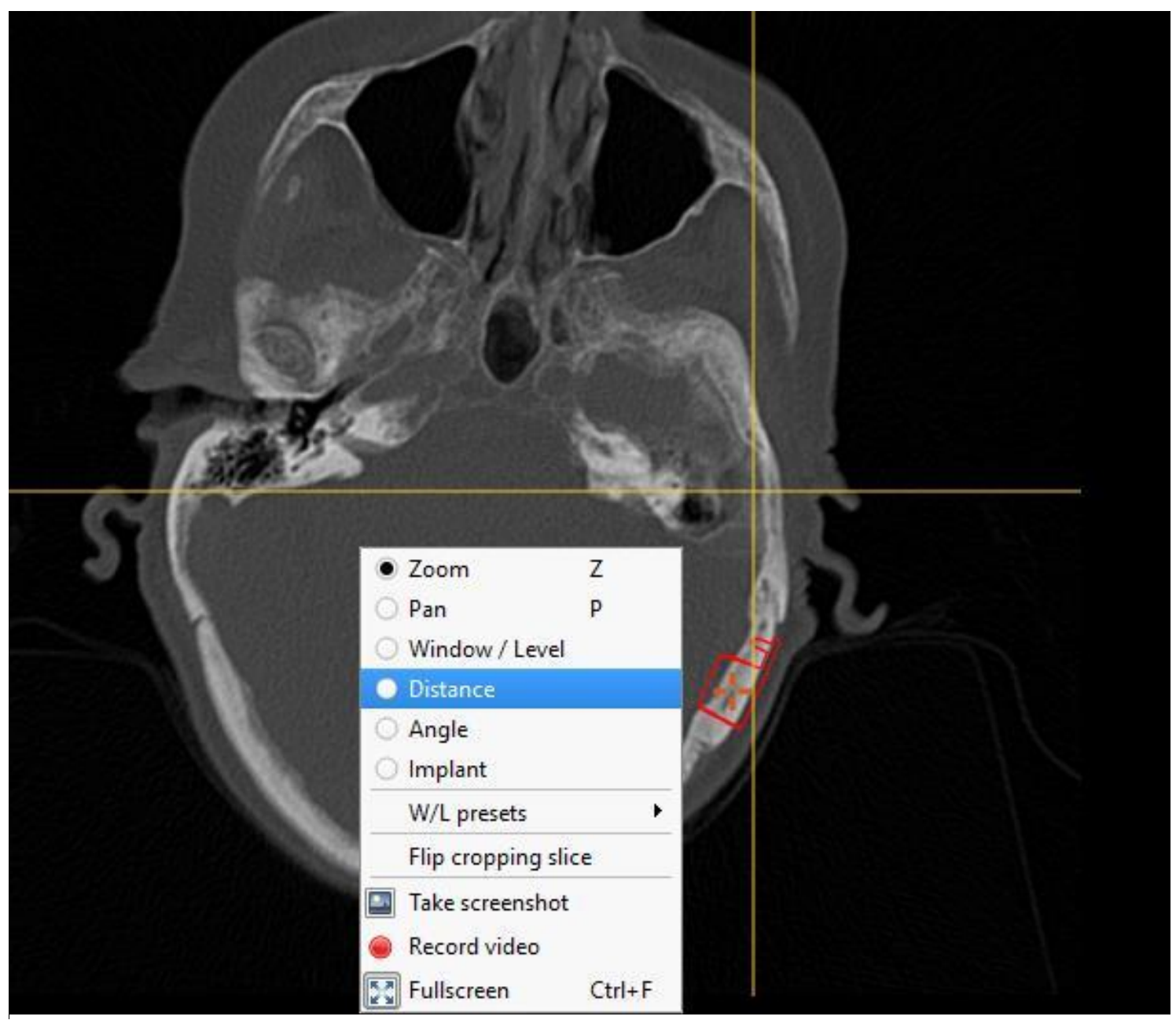

Abbildung 10: 2D Menü des BBFastView. Dieses Menüfenster lässt sich mit der rechten Computermaustaste öffnen. Einfache Befehle werden hiermit ausführbar.

Möchte man sich im 3D View bewegen, ist eine eigene Optionenrubrik am rechten Fensterrand dargestellt. Befehle wie Zoom und Fullscreen-Einstellung, aber auch Drehungen sowie direkte Einstellungen auf die linke und rechte Schädelseite sind möglich. Über die rechte Taste der Computermaus kann auch im 3D-Fenster ein eigenes Menü aufgerufen werden (siehe Abbildung 11). 


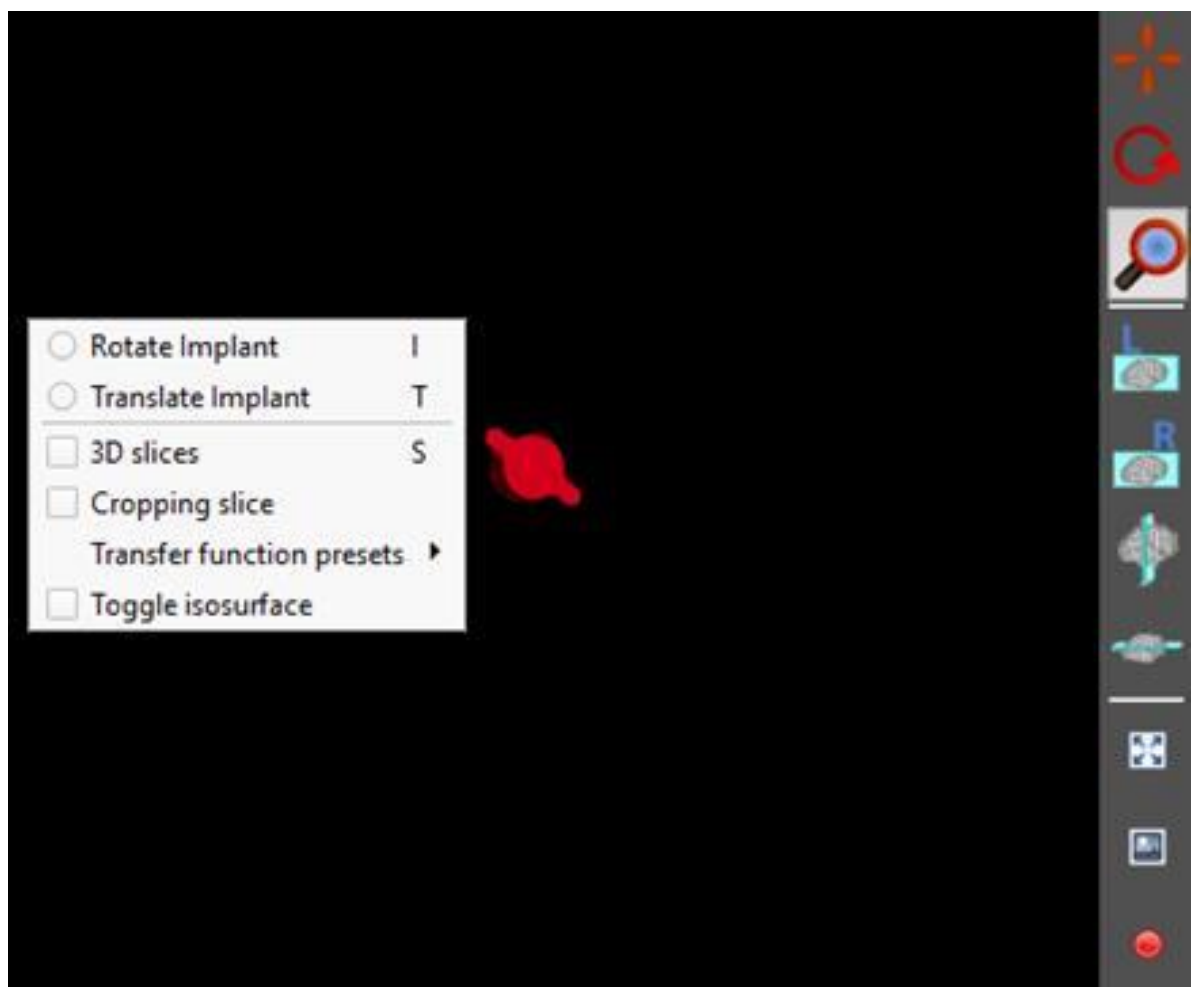

Abbildung 11: 3D Menü des BBFastView. Dieses Bedienfeld lässt sich in dem 3D-Fenster mit der rechten Maustaste öffnen. Es ermöglicht wichtige Funktionen wie das Cropping slice, um auch den intrakranialen Sitz des Implantates überprüfen zu können. In der Bildmitte sieht man in roter Farbe das template des Aktuators; zum Zeitpunkt der Bildschirmaufnahme sind keine 3DRekonstruktionen aktiviert. Am rechten Bildrand zeigt sich die für das 3DFenster eigene Optionenrubrik.

Eine Rotation und/oder Translation des Implantats im rekonstruierten 3D-Modell wurde innerhalb dieser Arbeit nicht durchgeführt. Die Bewegungen im 3D-Fenster waren häufig zeitverzögert, sodass dieses Fenster erst nach Implantation zur Kontrolle genutzt wurde. Als hilfreich hat sich die Funktion Cropping slice erwiesen, mit welcher in Kombination des 2D-Menüs Crop using this slice verschiedene Anteile des rekonstruierten Schädels entfernt werden können. Dies ermöglicht Einsicht nach intrakranial, wie es beispielsweise für die Auswertung der Flächen der jeweiligen Duraimpression notwendig gewesen ist.

\subsubsection{PACS Workstation}

PACS ist die gebräuchliche Abkürzung für ein System zur Verwaltung und Speicherung medizinischer Bilder und Daten und steht für Picture Archiving and Communication System (ITZ Medicom 2017). Dieses System wird in der Universitätsmedizin Göttingen angewandt, um abteilungsübergreifend Bilder und Daten speichern und abrufen zu können. Die PatientenCTs dieser Studie wurden aus dem PACS extrahiert. Als Datenformat wird das international gültige DICOM (Digital Imaging and COmmunications in Medicine) Format verwendet 
(www.itz-medi.com 2012), welches auch von der genutzten Planungssoftware BBFastView unterstützt wird.

\subsubsection{Statistica}

Um die statistische Auswertung durchzuführen wurde die Software STATISTICA, Version 12.5 (StatSoft, Tulsa, Oklahoma, USA) genutzt. Bei den vorgestellten Daten handelt es sich um Durchschnittsmesswerte \pm Standardabweichung.

Ein t-Test wurde verwendet, um geschlechtsspezifische, statistisch relevante Unterschiede sowie Unterschiede zwischen den drei Implantationslokalisationen festzustellen. Während der Tests wurde ein Wahrscheinlichkeitswert von $\alpha<0,05$ als statistisch signifikant festgelegt.

\subsection{Parameter und Messungsmethoden}

Die drei Implantationslokalisationen wurden auf bestimmte Bewertungskriterien hin untersucht, die im Folgenden detailliert vorgestellt werden:

Nachdem das in 0,6 mm Feinschicht vorliegende Schädel-CT auf Vollständigkeit und Artefakte geprüft worden war, konnte mit Hilfe der Planungssoftware BBFastView die virtuelle Implantation begonnen werden. Für jede der drei Implantationslokalisationen, retrosigmoidal, parietal und im Mastoidresiduum, wurde eine bestmögliche Implantation des BCFMTs gefunden. Diese beruhte auf einer möglichst geringen Duraimpression, der Schonung der Sinus sigmoideus und transversus, sowie einem möglichst geringen Abstand zur Cochlea. Weiter wurde der Aktuator möglichst auf eine Weise platziert, dass die flügelartigen Schraubenösen bündig mit der Schädelkalotte zum Liegen kamen. Bei Platzierung im Mastoidresiduum war zusätzlich darauf zu achten, dass das Implantat nicht zu den pneumatisierten Räumen wie Gehörgang, Paukenhöhle und/oder der chirurgisch angelegten pneumatisierten Höhle frei zu liegen kam. Es wäre denkbar, dass mögliche Entzündungen durch Entwicklung eines Biofilms auf dem Fremdmaterial unterhalten werden würden.

Der implantierte Teil der Bonebridge besteht aus einer Spule, einem Demodulator sowie dem BC-FMT. Der BC-FMT ist der Aktuator der Bonebridge, misst 8,7 $\mathrm{mm}$ in der Tiefe sowie 15,8 $\mathrm{mm}$ in der Breite und wird durch zwei Schrauben mit einer Eindringtiefe von jeweils 3,9 mm im Schädelknochen befestigt. Der Abstand zwischen den Schraubenösen beträgt 23,8 $\mathrm{mm}$ (siehe Abbildung 12, A). Weiter sind sogenannte BCI-lifts anwendbar, die die Eindringtiefe des Masseschwingers in die Schädelkalotte verringern können (siehe Abbildung 12, B).

Der zylinderförmige Aktuator, mit den die Schrauben tragenden Flügeln, kann bei Anwendung der Planungssoftware BBFastView als template geladen werden und intuitiv mit der Computermaus im Schädel-CT positioniert werden. 
A

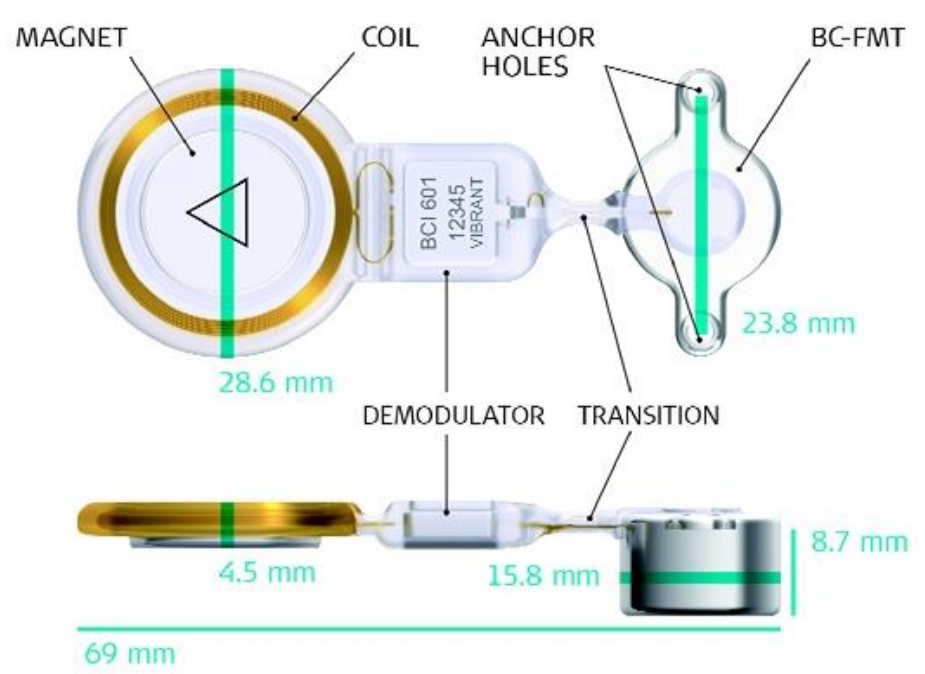

B

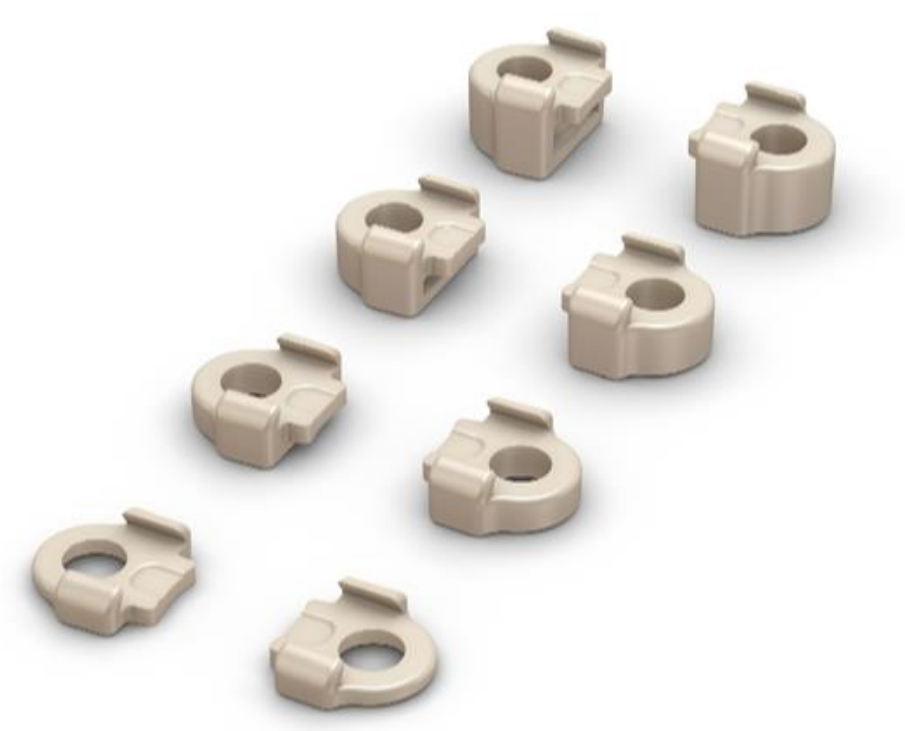

Abbildung 12: Bone Conduction Implant (BCI). In $\mathbf{A}$ ist das BCI sowohl von oben als auch von der Seite mit den Komponenten Spule (coil), Demodulator und BC-FMT gezeigt. Spule $(4,5 \mathrm{~mm})$ und Demodulator liegen der Schädelkalotte auf und sind von der Kopfschwarte bedeckt. Der BC-FMT ist 15,8 $\mathrm{mm}$ breit und $8,7 \mathrm{~mm}$ tief, sodass ein entsprechendes Bett in die Schädelkalotte gebohrt werden muss. Befestigt ist der implantierte Teil mit zwei Schrauben mit einer effektiven Eindringtiefe von jeweils 3,9 $\mathrm{mm}$ und einer Gesamtlänge von $6 \mathrm{~mm}$. In $\mathbf{B}$ sind die Abstandshalter (BCI-lifts) dargestellt, die mit einer Stärke von 1 bis 4 mm die Eindringtiefe des Masseschwingers reduzieren können. Illustration mit freundlicher Genehmigung der Fa. MED-EL, Innsbruck, Österreich (modifiziert). 


\subsubsection{Beurteilungskriterien für retrosigmoidale und parietale Lokalisation}

Um nach bestmöglicher virtueller Implantation die Lokalisation beurteilen zu können, wurden folgende Messparameter definiert:

Die Dicke der Schädelkalotte mittig über dem BC-FMT template ist ein wichtiges Kriterium zur Beurteilung einer der alternativen Implantationslokalisationen. Hierbei ist unmittelbar ersichtlich, ob bei Implantation die Dura tangiert oder imprimiert werden könnte. Zur Durchführung der Messung wird im axialen Fenster der Software gearbeitet und diejenige CT-Schicht ausgewählt, in welcher das template als Zylinder eine möglichst rechteckige, gleichmäßige Form aufweist. Dies lässt auf den Mittelpunkt des Implantats schließen. Anschließend wird mit dem Befehl Distance der Software ein Messpunkt an den äußeren Rand und ein zweiter an die intrakraniale Begrenzung der Schädelkalotte gelegt. Der Distanzwert wird unmittelbar vom Programm angezeigt und die gemessene Strecke in grün markiert (siehe Abbildung 13).

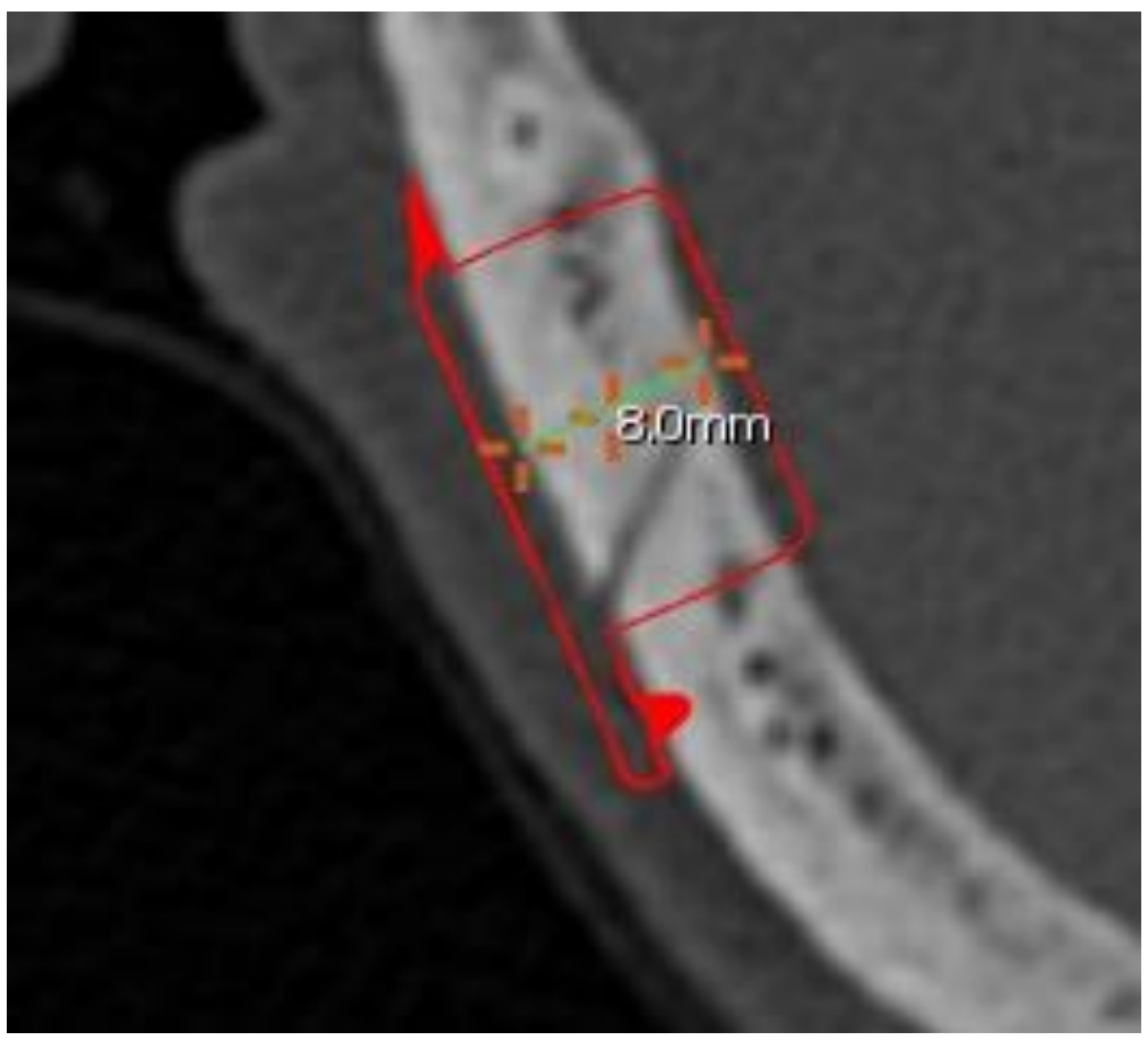

Abbildung 13: Dicke der Schädelkalotte mittig über dem BC-FMT. Im axialen Schnittbild ist die knöcherne Schädelkalotte zu sehen, über welcher das in roter Umrandung dargestellte template des BC-FMTs platziert ist. Die Messung erfolgt von der Außenseite der Schädelkalotte bis zur Innenseite der Schädelkalotte (grüne Messlinie) mittig über dem Zylinder (rechteckige Darstellung des FMTs). Bei diesem Patienten beträgt die Dicke der Schädelkalotte mittig über dem Aktuator $8 \mathrm{~mm}$. 
Weiter wird die Stärke der knöchernen Schädeldecke bei den jeweiligen Schraubenösen betrachtet. Hier werden bei Implantation Schrauben mit einer effektiven Eindringtiefe von 3,9 $\mathrm{mm}$ verwendet. Folglich würde ein Messwert von weniger als $4 \mathrm{~mm}$ auf eine mögliche Verletzung der Dura durch das scharfe Schraubenende hinweisen. In Abbildung 14 ist eine Beispielmessung dargestellt, in der die Schraube des templates in der Planungssoftware dargestellt ist. Die Messung erfolgt in derjenigen CT-Schicht, in der die Schraubenschablone ihre maximale Länge erreicht. Gemessen wird die Dicke der Schädelkalotte jeweils im Bereich der ventral und der dorsal gelegenen Schraube (Abbildung 14 und Abbildung 15).

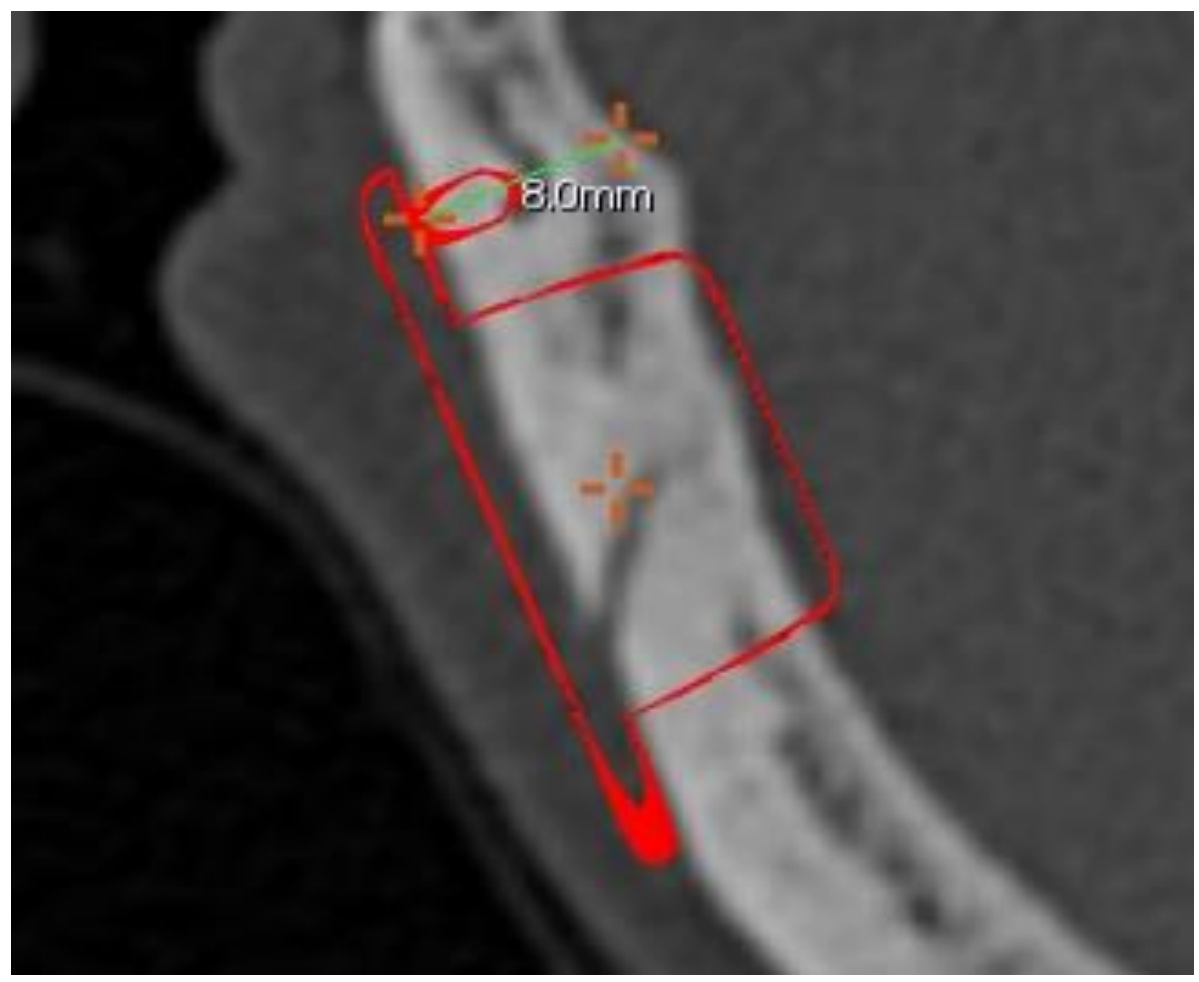

Abbildung 14: Dicke der Schädelkalotte bei Schraubenöse 1. Zu erkennen ist die Schädelkalotte mit platzierter Schablone. In diesem Anschnitt der Schablone ist der BC-FMT und eine Schraube dargestellt (ventrale Schraube). Die Messung erfolgt über die Funktion Distance, indem ein Messpunkt nach außen und ein Messpunkt nach innen an die Schädelkalotte gelegt wird. Im Beispiel beträgt die Dicke der Schädelkalotte bei Schraubenöse $18 \mathrm{~mm}$. Die Schraube zeigt in dieser CT-Schicht ihre maximale Länge. Die Bildgebung liegt im axialen Fenster vor. 


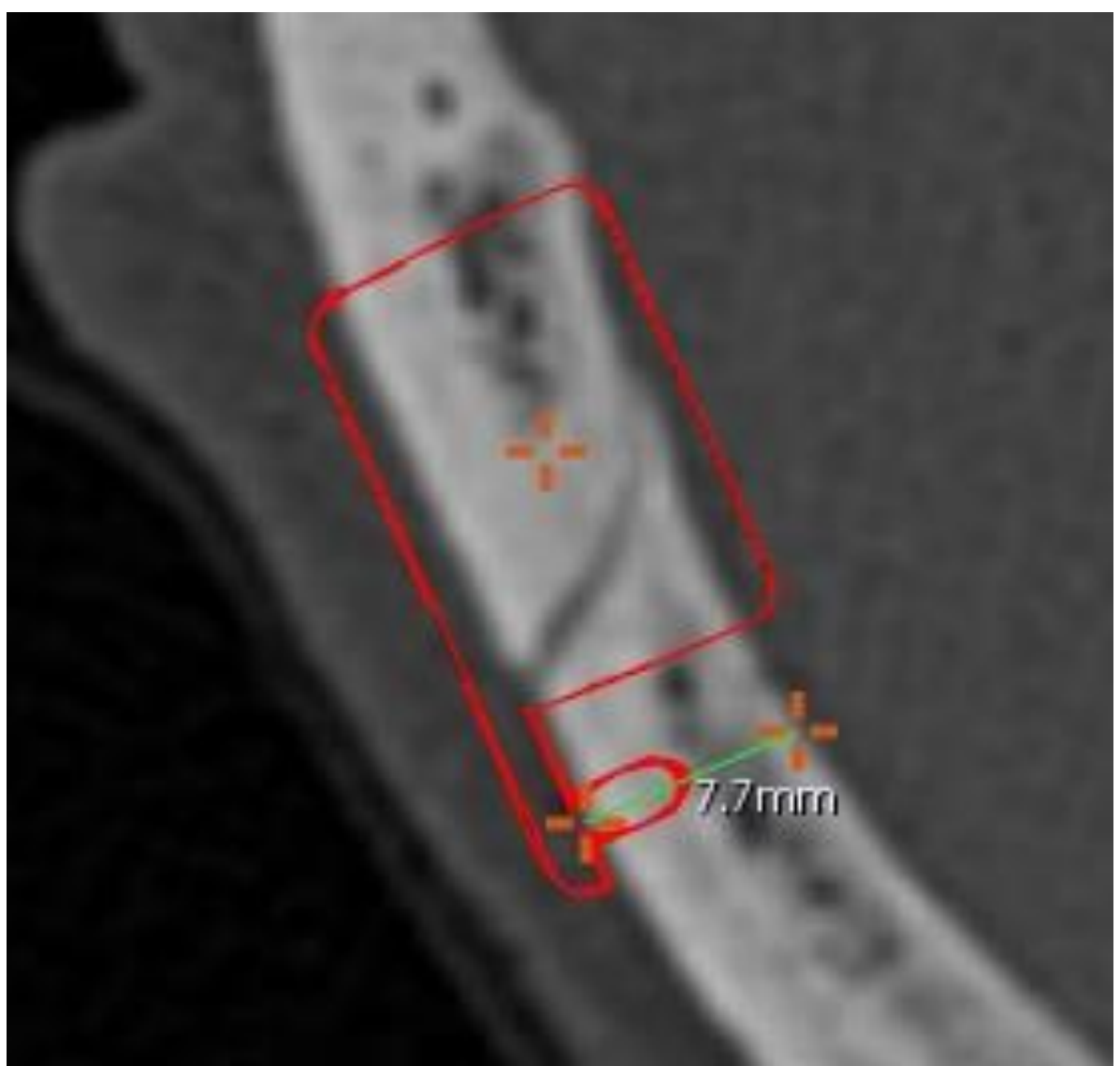

Abbildung 15: Dicke der Schädelkalotte bei Schraubenöse 2. Zu erkennen ist die Schädelkalotte mit platzierter Schablone. In diesem Anschnitt der Schablone ist der BC-FMT und eine Schraube dargestellt (dorsale Schraube). Die Messung erfolgt über die Funktion Distance, indem ein Messpunkt nach außen und ein Messpunkt nach innen an die Schädelkalotte gelegt wird. Im Beispiel beträgt die Dicke der Schädelkalotte bei Schraubenöse $27,7 \mathrm{~mm}$. Die Schraube zeigt in dieser CT-Schicht ihre maximale Länge. Die Bildgebung liegt im axialen Fenster vor. 


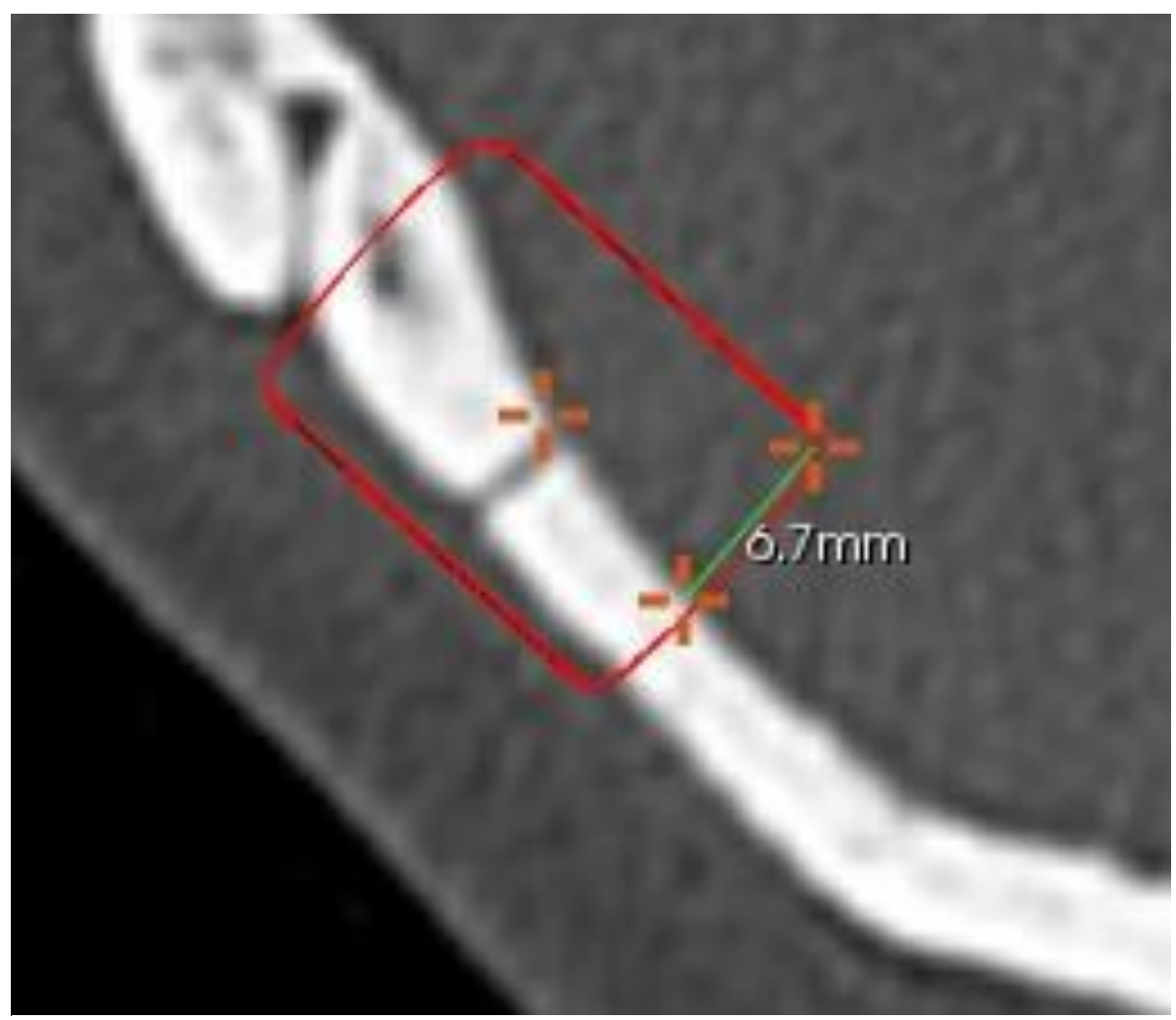

Abbildung 16: Maximale Duraimpression. Dargestellt ist die Schädelkalotte in einem Bereich verhältnismäßig geringer Dicke. Das in roter Farbe dargestellte BC-FMT template ragt deutlich nach intrakranial. Die Messung im axialen Fenster beschreibt eine Duraimpression von $6,7 \mathrm{~mm}$.

Ein weiteres Kriterium bei der Beurteilung einer möglichen Implantation im retrosigmoidalen oder parietalen Bereich, ist das Ausmaß einer etwaigen Duraimpression (siehe oben Abbildung 16). Die maximale Duraimpression nach bestmöglicher virtueller Implantation des templates wird mittels des Softwarebefehls Distance gemessen; dies erfolgt in derjenigen CT-Schicht, in der die Impression für den individuellen Patientenfall als maximal erkannt wird. Abbildung 16 zeigt eine Momentaufnahme aus der Software BBFastView. Der Patient hat eine deutlich dünnere Schädeldecke als das BC-FMT tief ist. Folglich käme es bei einer Implantation in dieser Region zu einer Duraimpression von 6,7 mm. Komplikationen wie Liquoraustritt oder epidurale Hämatome wären theoretisch denkbar. Auch unter Verwendung der Abstandshalter, BCI-lifts, wäre in diesem Fall der Kontakt zur Dura nicht verhindert, da diese die Eindringtiefe um maximal $4 \mathrm{~mm}$ reduzieren können.

Zur weiteren Beurteilung der Lokalisation wurde neben der Tiefe auch die Fläche der jeweiligen Duraimpression einer virtuellen Implantation betrachtet. Die Graduierung erfolgt in vier Stufen, die wie folgt definiert wurden:

Grad 0: Die Implantatbasis liegt nicht frei. Es ist ein gänzlicher Knochenüberzug von intrakranial gegeben. Implantationen, die in der jeweiligen Position mit dem Grad 0 bewertet wurden, beeinträchtigen die Dura nicht. 
Grad 1: Die Implantatbasis liegt zu weniger als der Hälfte frei. Die Knochendecke von intrakranial ist nicht mehr geschlossen. Die Basis des Implantats kommt zwangsläufig mit der Dura des Patienten in Kontakt (siehe Abbildung 17).

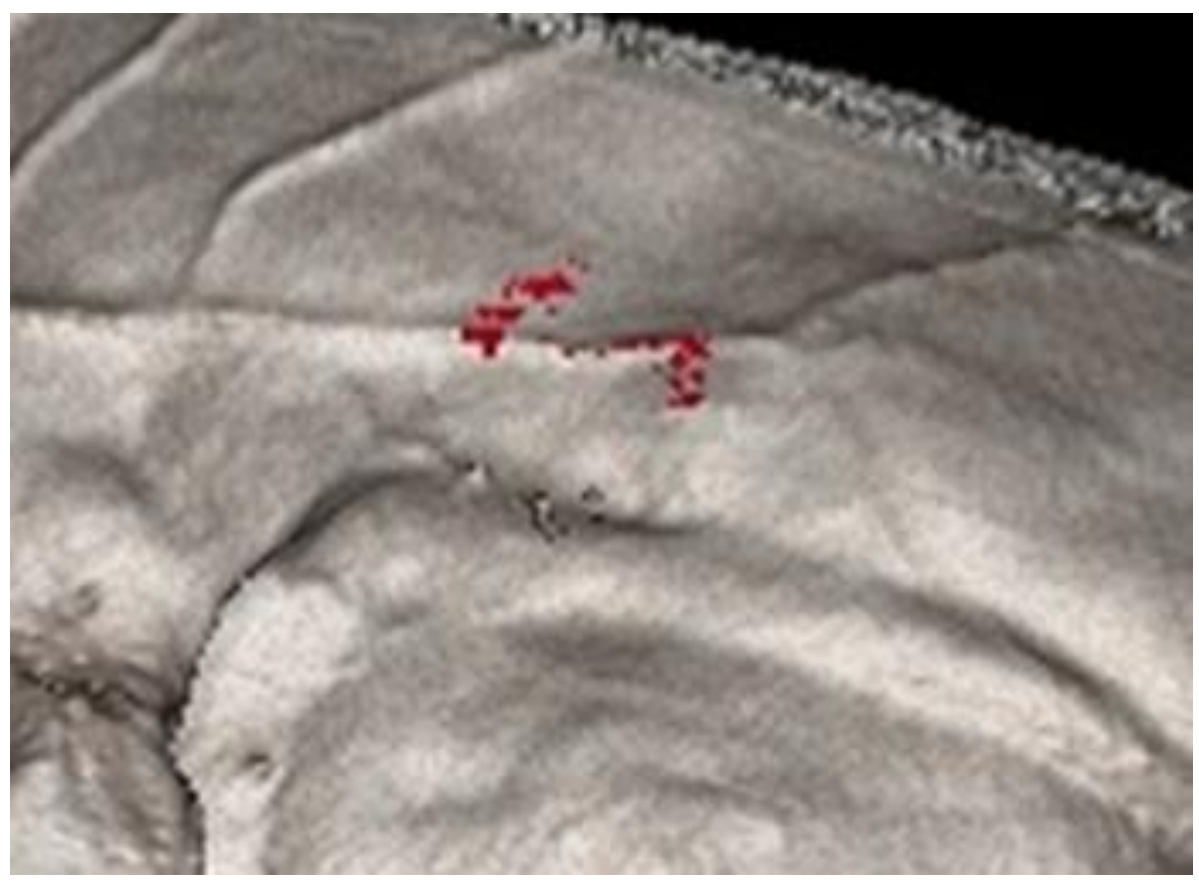

Abbildung 17: 3D-Visualisierung in BBFastView, Fläche der freiliegenden Implantatbasis Grad 1. Es ist ein Teilausschnitt der Schädelkalotte von intrakranial zu sehen. In roter Farbe ist die Basis des von extrakranial in die Schädelkalotte implantierten BC-FMTs zu erkennen. In kaudaler Richtung ist der Verlauf der Sinus sigmoideus und transversus durch ihren Sulcus zu erkennen. Dargestellt ist eine beispielhafte Implantation im parietalen Bereich. Die Knochendecke bedeckt die Implantatbasis nicht mehr vollständig (Grad 1).

Grad 2: Die Implantatbasis ist zu mehr als der Hälfte freiliegend. Die Knochendecke ist über der Basis des Implantats größtenteils aufgebrochen. Die Basis des Implantats steht mit der Dura in Kontakt (siehe Abbildung 18). 


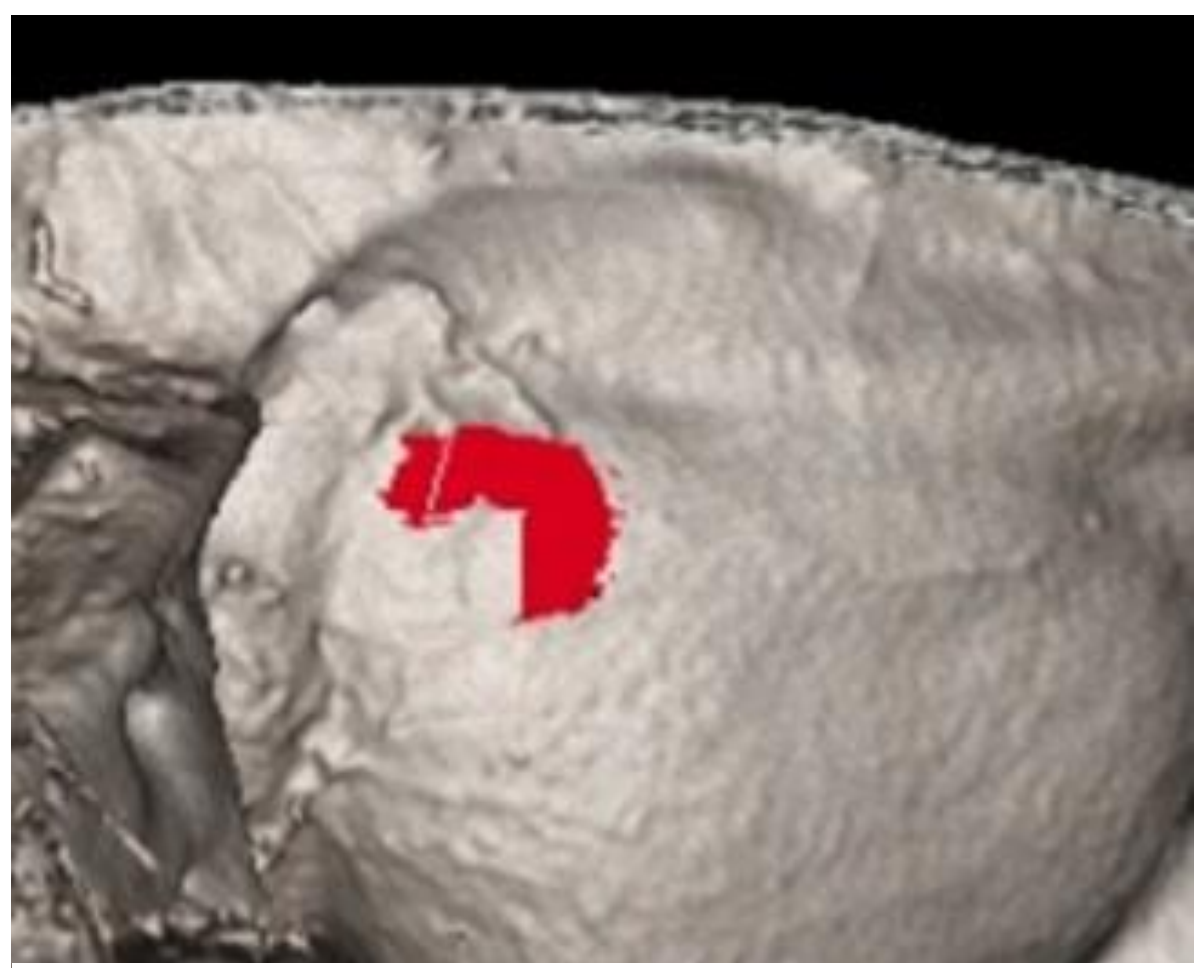

Abbildung 18: 3D-Visualisierung in BBFastView, Fläche der freiliegenden Implantatbasis Grad 2. Es ist ein Teilausschnitt der Schädelkalotte von intrakranial zu sehen. In roter Farbe ist die Basis des von extrakranial in die Schädelkalotte implantierten BC-FMTs zu erkennen. Nach kranial ist der Sulcus der Sinus transversus und sigmoideus zu erkennen. Es handelt sich um eine beispielhafte Implantation im retrosigmoidalen Bereich. Die Knochendecke ist über der Basis des Implantats größtenteils aufgebrochen (Grad 2).

Grad 3: Die Implantatbasis liegt nach intrakranial vollständig frei. Es ist keine Knochendecke über der Basis vorhanden. Das Implantat steht in Kontakt mit der Dura und es ist eine Impression wahrscheinlich (siehe Abbildung 19). 


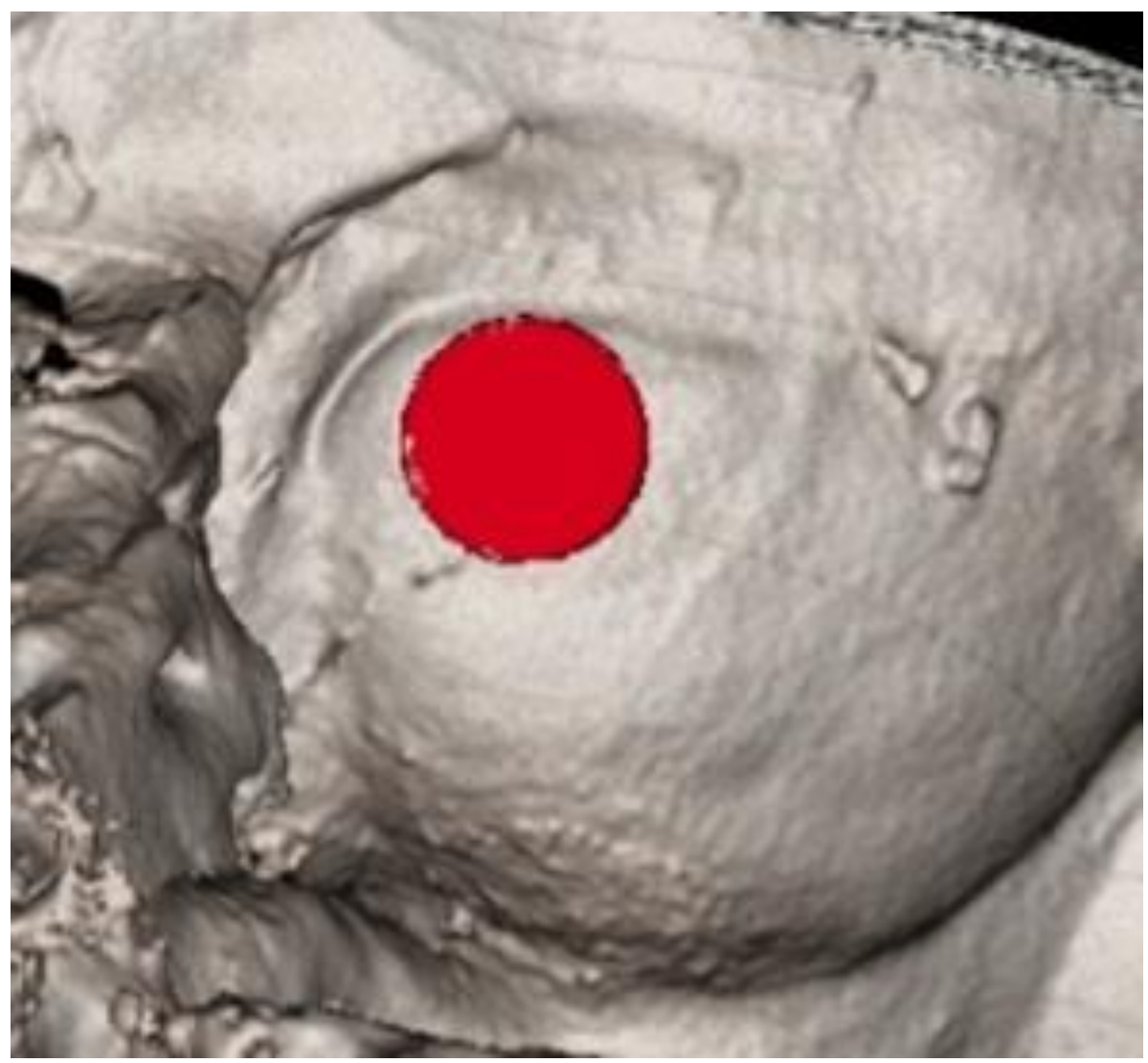

Abbildung 19: 3D-Visualisierung in BBFastView, Fläche der freiliegenden Implantatbasis Grad 3. Es ist ein Teilausschnitt der Schädelkalotte von intrakranial zu sehen. In roter Farbe ist die Basis des von extrakranial in die Schädelkalotte implantierten BC-FMTs zu erkennen. Nach kranial ist der Sulcus Sinus transversus und sigmoideus zu erkennen. Es handelt sich um eine beispielhafte Implantation im retrosigmoidalen Bereich. Über der Basis des Implantats ist keine Knochensubstanz zu erkennen (Grad 3).

Für die bestmögliche Leistungsfähigkeit des Knochenleitungshörsystems und verlustärmste Schallübertragung vom Aktuator über den Schädelknochen zur Cochlea sollte der Abstand zwischen Mitte des zylinderförmigen Aktuators und der Cochlea möglichst kurz gehalten werden. Eine Abnahme der Vibrationsamplitude mit zunehmender Entfernung zur Cochlea ist bekannt (Eeg-Olofsson et al. 2008). Der Mittelpunkt des Zentrums des Aktuators ist der mittlere Abstand zu den beiden Implantatschrauben, die den Zylinder im Schädelknochen fixieren und letztlich die Vibration auf den Knochen übertragen. Bei der Messung des Abstands mit Hilfe der Software wird der erste Messpunkt mittig am BC-FMT gesetzt (siehe Abbildung 20, A) und anschließend die CT-Schicht ausfindig gemacht, in der die Cochlea abgebildet ist. Zwischen zweiter und dritter Windung der Cochlea wird der zweite Messpunkt gesetzt (siehe Abbildung 20, B), sodass die Software den entsprechenden Wert der Strecke anzeigt. Die Messung findet ebenfalls in axialer Bildgebung statt. 

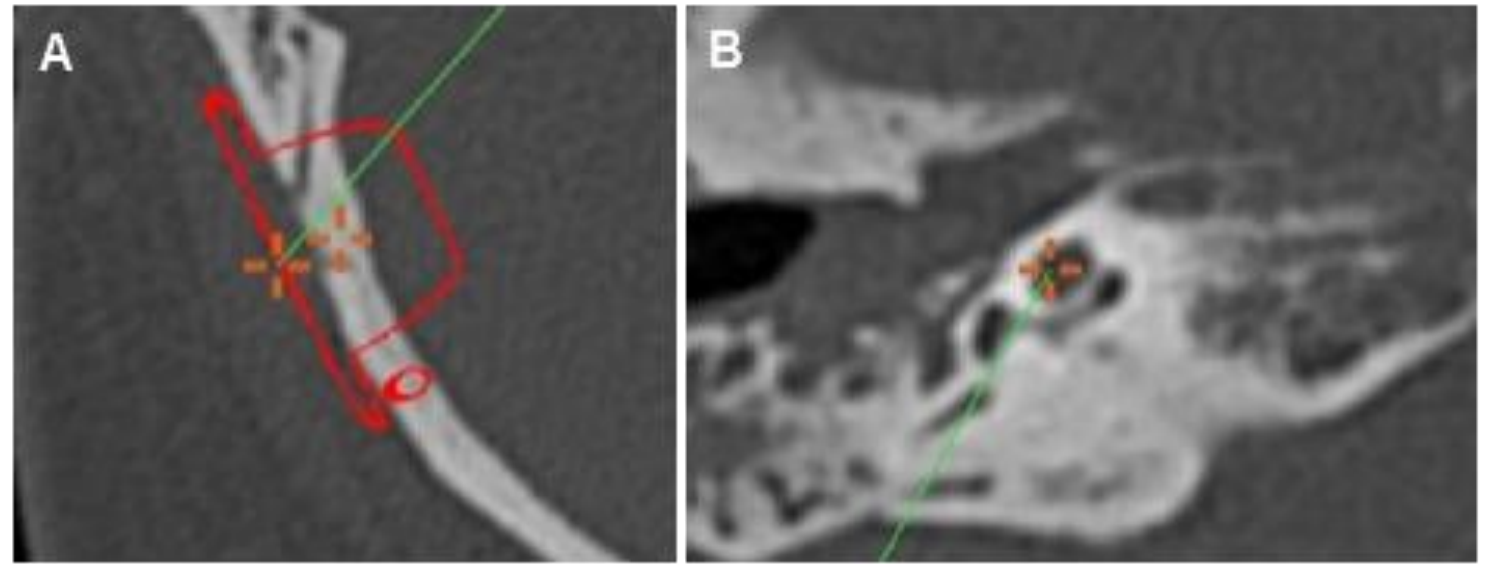

Abbildung 20: Minimale Entfernung des FMT-Zentrums zur Cochlea. A Die Schädelkalotte mit dem in Rot dargestelltem template des BC-FMTs ist zu erkennen. Zur Bestimmung der Entfernung des FMT zur Cochlea wird der erste Messpunkt im mittleren Abstand zu den beiden Schraubenösen gesetzt. In Grün ist die Strecke in Richtung Messpunkt 2 (Cochlea, B) dargestellt. B Als pyramidenförmige Aufhellung ist hier die Cochlea zu sehen. Die erste Windung liegt als hantelförmige Basis unter der kugelförmigen Aufhellung, die der zweiten und dritten Windung der Cochlea entspricht. Zwischen zweiter und dritter Windung ist der zweite Messpunkt gesetzt. In grün ist die Strecke in Richtung Messpunkt 1 (FMT-Zentrum) dargestellt. Die Messung findet im axialen Fenster statt.

Als weiteres mögliches Kriterium - um die alternativen Implantatlokalisationen zu bewerten - wurde der Winkel zwischen dem BC-FMT-Zentrum zur Frankfurter Horizontalen gemessen. Die Frankfurter Horizontale wird definiert als die Verbindung vom tiefsten Punkt der knöchernen Orbita zum kranialsten Punkt des knöchernen Gehörgangs (Paulsen und Waschke 2010). Somit ergeben sich drei Punkte im Raum, die ermittelt werden müssen, um den Winkel mit Hilfe des Befehls Angle der Software messen zu können: FMTZentrum, kranialster Punkt des knöchernen Gehörgangs sowie kaudalster Punkt der Orbita.

Der kaudalste Punkt der Orbita wird im axialen Fenster ermittelt, indem man sich von kaudal nach kranial langsam durch die Schichten des CTs arbeitet. Es wird der Schichtbereich ausgewählt, in dem sich die Knochenlamelle der Orbita eben noch als zusammenhängende Struktur zeigt, bevor sich das Bild im Weichgewebe des Augapfels verliert (siehe Abbildung 21, A). Mit Hilfe des Fadenkreuzes wird dieser Punkt markiert und in der sagittalen Bildgebung kontrolliert (siehe Abbildung 21, B). Der kranialste Punkt des knöchernen Gehörgangs wird ebenfalls im axialen Fenster ermittelt. Die Schichten werden wieder von kaudal nach kranial betrachtet, um den Punkt zu ermitteln, an dem sich die laterale Knochenlamelle über dem pneumatisierten Gehörgang schließt (siehe Abbildung $21, \mathrm{C})$. 

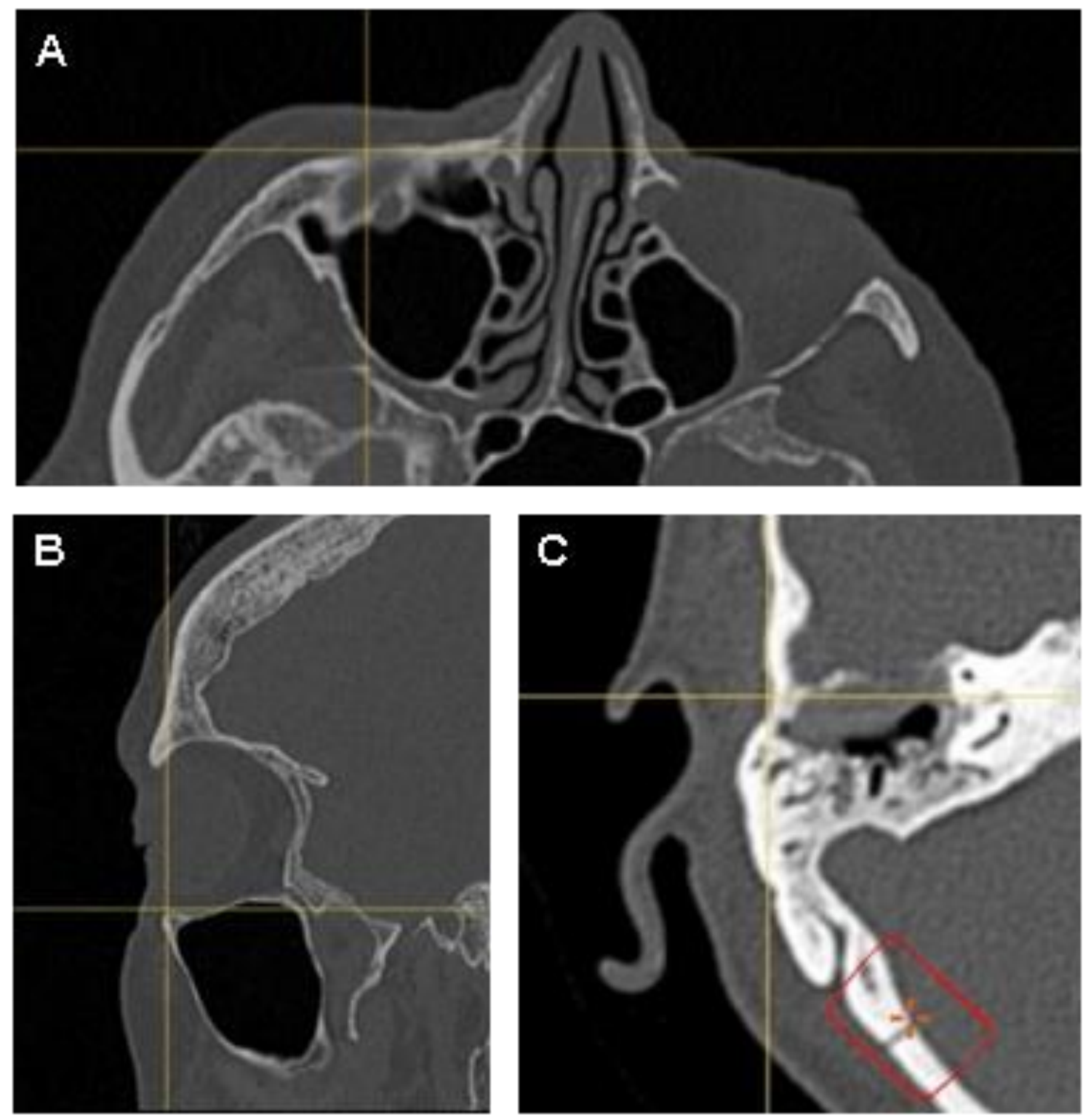

Abbildung 21: Winkel zwischen FMT-Zentrum und Frankfurter Horizontale. A Es ist der ventrale Teil eines Schädel-CTs in axialer Bildgebung dargestellt. Das Fadenkreuz markiert den Punkt an dem sich die Knochenlamelle des kaudalen Orbitarandes schließt, demnach den kaudalsten Punkt. B Es ist eine sagittale Darstellung eines Schädel-CTs gezeigt. Der linke Bildrand entspricht der ventralen Patientenseite. Als nach ventral offene Halbkugel zeigt sich die Verschattung im Sinne der knöchernen Orbita. Mit Hilfe des Fadenkreuzes ist der kaudalste Punkt der Orbita markiert, dies als ein Punkt von der zu ermittelnden Frankfurter Horizontalen. C In axialer Bilddarstellung ist die rechte Ohr- und Felsenbeinregion des Patienten ersichtlich. In roter Umrandung ist das template des Aktuators abgebildet. Mit dem Fadenkreuz wurde der Punkt markiert, an dem sich die laterale Knochenlamelle über dem pneumatisierten Gehörgang schließt.

Bei der Winkelmessung mit Hilfe des Softwarebefehls Angle wird mit dem kaudalsten Orbitapunkt begonnen. Es folgt das Auffinden des kranialsten Punktes des äußeren, knöchernen Gehörganges und damit die Vervollständigung der Frankfurter Horizontale. AbschlieBend wird die Frankfurter Horizontale mit dem Zentrum des FMTs verbunden. Die Software zeigt nach Auffinden des dritten Punktes den Wert des Winkels automatisch an. 
Ein weiterer Beurteilungsaspekt ist die Dicke der Kopfschwarte über der Spule, sodass eine suffiziente Signalübertragung vom Audioprozessor auf die Spule erfolgen kann. Weiter hat die Dicke des Epikraniums Einfluss auf den magnetischen Halt zwischen externem Audioprozessor und Spule. Nach den Herstellerinformationen sind bei einer Stärke der Kopfschwarte bis zu $7 \mathrm{~mm}$ keine Beeinträchtigungen in der Signalübertragung zu erwarten. Des Weiteren ist die Lage der Spule abhängig vom gewählten Abknickungswinkel im Bezug zum FMT. Je nach Winkel verändere sich die Strecke zwischen FMT-Zentrum und SpulenZentrum (Fa. MED-EL 2012) (siehe Abbildung 22).

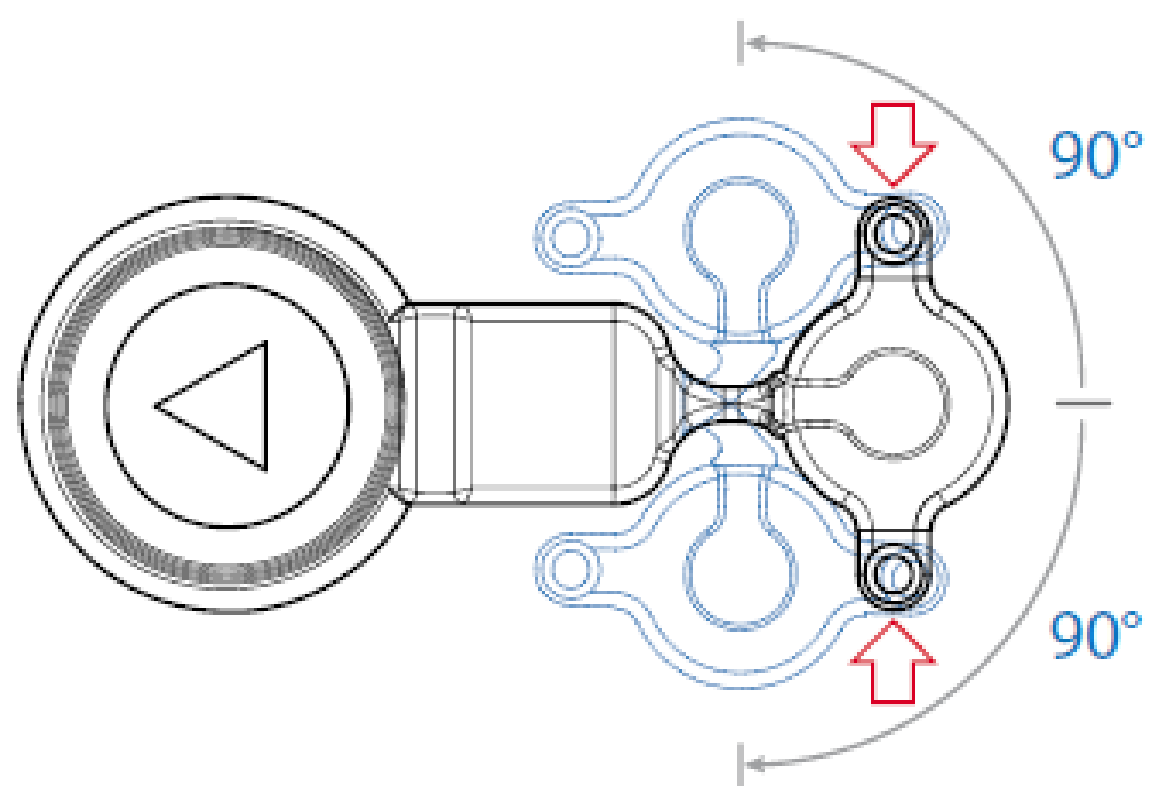

Abbildung 22: Abknickungswinkel des FMT zur Spule. Die Skizze der Bonebridge verdeutlicht den Bewegungsradius des FMTs gegenüber der Spule. Bis max. $90^{\circ}$ kann das Gerät in beide Richtungen gebogen werden. Die Länge der Bonebridge verändert sich dementsprechend. Illustration mit freundlicher Genehmigung der Firma MED.EL.

$0^{\circ}$ : Länge der Bonebridge beträgt $46 \mathrm{~mm}$ von Mitte des FMTs zu Mitte der Spule $45^{\circ}$ : Länge der Bonebridge beträgt $42 \mathrm{~mm}$ von Mitte des FMTs zu Mitte der Spule $90^{\circ}$ : Länge der Bonebridge beträgt $37 \mathrm{~mm}$ von Mitte des FMTs zu Mitte der Spule

In der vorliegenden Studie wurde der Winkel zwischen FMT und Spule von $45^{\circ}$ festgelegt und somit der Abstand von $42 \mathrm{~mm}$ zwischen FMT-Mittelpunkt und Spulenmittelpunkt bei der Bestimmung der Dicke der Kopfschwarte über der Spule herangezogen. Zu Beginn wird der Mittelpunkt der Spule im axialen Fenster bestimmt. Vom FMT-Zentrum werden $42 \mathrm{~mm}$ in Richtung dorsokranial ausgewählt (siehe Abbildung 23, A) um den Sitz des Audioprozessors möglichst verdeckt und an physiologisch sinnvoller Position tragen zu können. Im Anschluss wird vom derart bestimmten Ort des Spulenmittelpunktes die Dicke der 
Kopfschwarte gemessen (siehe Abbildung 23, B). Beide Messungen werden mit der Distance Funktion der Software ausgeführt.
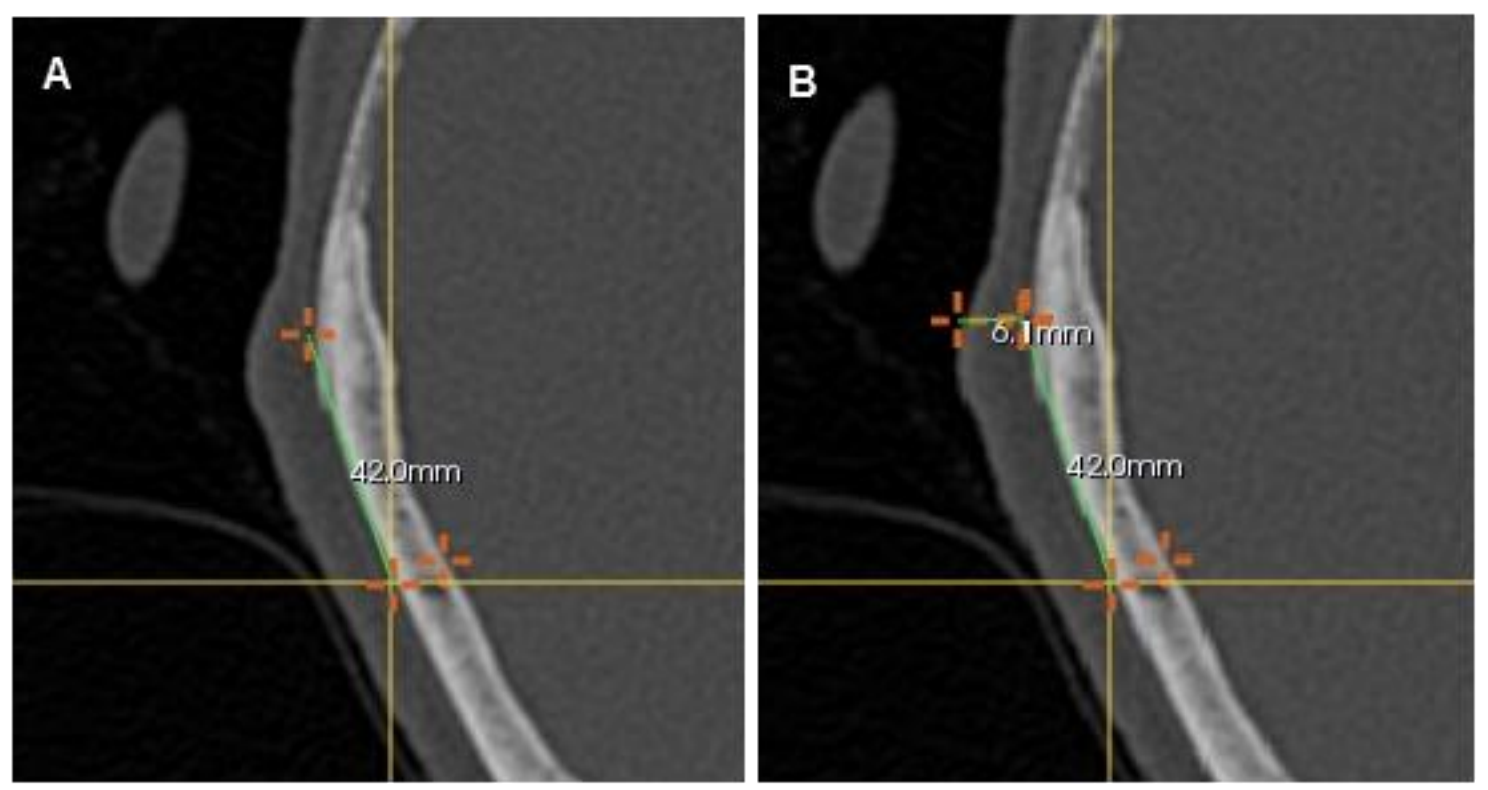

Abbildung 23: Dicke der Kopfschwarte über dem Mittelpunkt der Spule. Die CT's zeigen eine axiale Schicht und die Region kranial des rechten Ohres ist abgebildet. Die ovale, weichteildichte Struktur (jeweils oben links) entspricht der kranialen Ohrmuschelkante. Die obere Bildkante liegt ventral, die untere dorsal. A Vom Mittelpunkt des FMTs (Fadenkreuz/ Messpunkt 1) werden $42 \mathrm{~mm}$ in dorsokranialer Richtung vermessen und somit die Lage des Spulenmittelpunktes nach Implantation ermittelt (Messpunkt 2). B Über dem virtuell festgelegten Spulenmittelpunkt wird die Dicke der Kopfschwarte vermessen. Im Beispiel beträgt die Dicke der Kopfschwarte über dem Mittelpunkt der Spule 6,1 mm. 
Eine abschließende Messung fand jeweils über dem Mittelpunkt des FMTs statt, um auch dort die Dicke der Kopfschwarte beurteilen zu können (siehe Abbildung 24). Diese Messung wurde im axialen Fenster der Software durchgeführt.

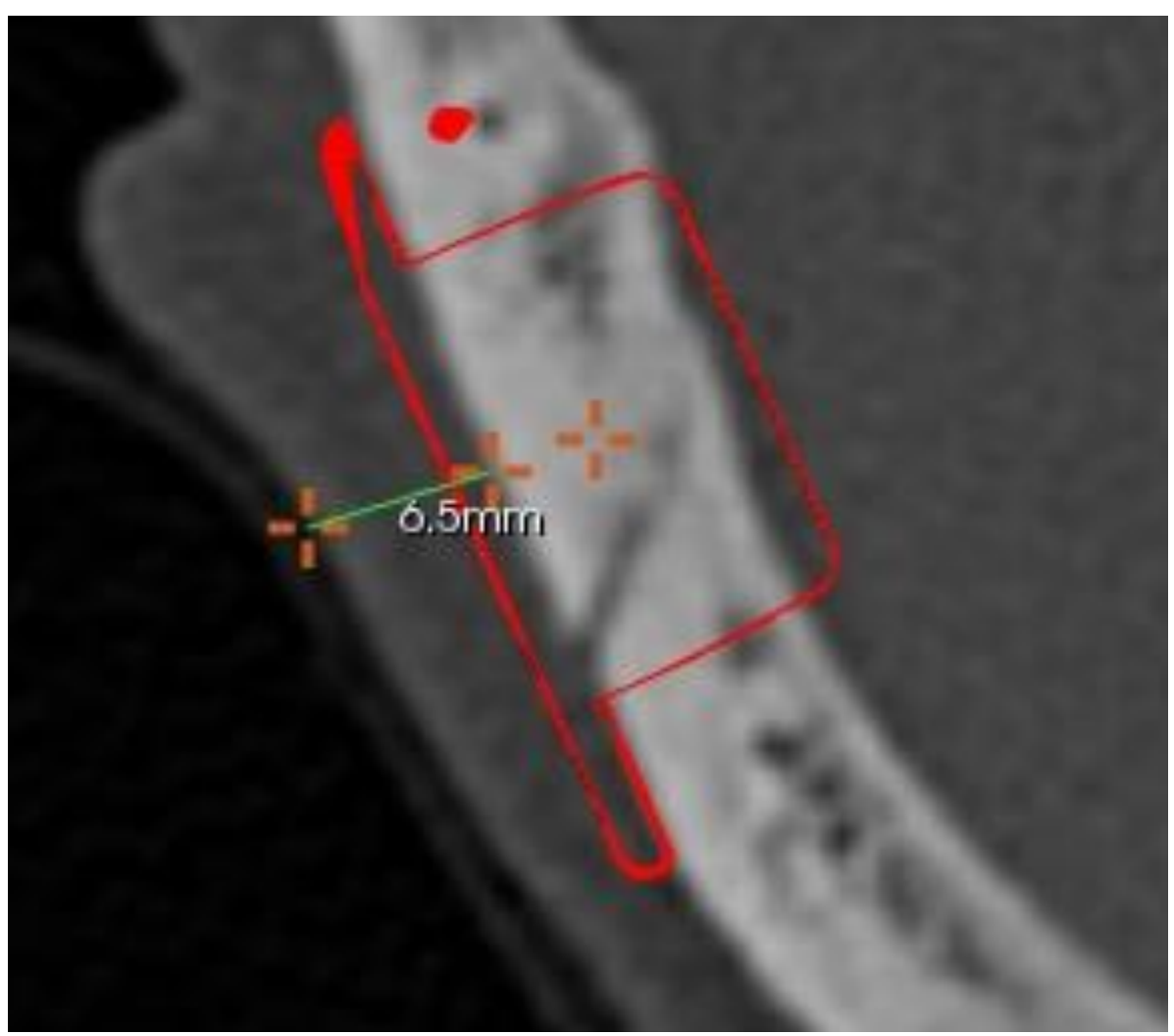

Abbildung 24: Dicke der Kopfschwarte über dem Mittelpunkt des FMTs. Im axialen Fenster der BBFastView-Software ist das bestmöglich implantierte template in roter Farbe zu sehen. Über dem Mittelpunkt des FMTs wurde die Dicke des Epikraniums vermessen. Im Beispiel beträgt diese 6,5 $\mathrm{mm}$.

\subsubsection{Beurteilungskriterien für die Implantation im Mastoidresiduum}

Um das Mastoidresiduum in Bezug auf eine noch mögliche Implantation beurteilen zu können, wurden teilweise andere Messungen durchgeführt als bei den retrosigmoidalen und parietalen Schädellokalisationen. Gemeinsame Kriterien der drei Implantationslokalisationen sind im Folgenden aufgeführt: minimale Entfernung des FMT-Zentrums zur Cochlea, Winkel des FMT-Zentrums zur Frankfurter Horizontalen, Dicke der Kopfschwarte über der Spule. Im Weiteren wurde für die mastoidale Platzierung der Kontakt zum nahegelegenen Sinus sigmoideus bestimmt und im Falle eines bestehenden Kontaktes auch die maximale Impression des Sinus sigmoideus gemessen (siehe Abbildung 25). 


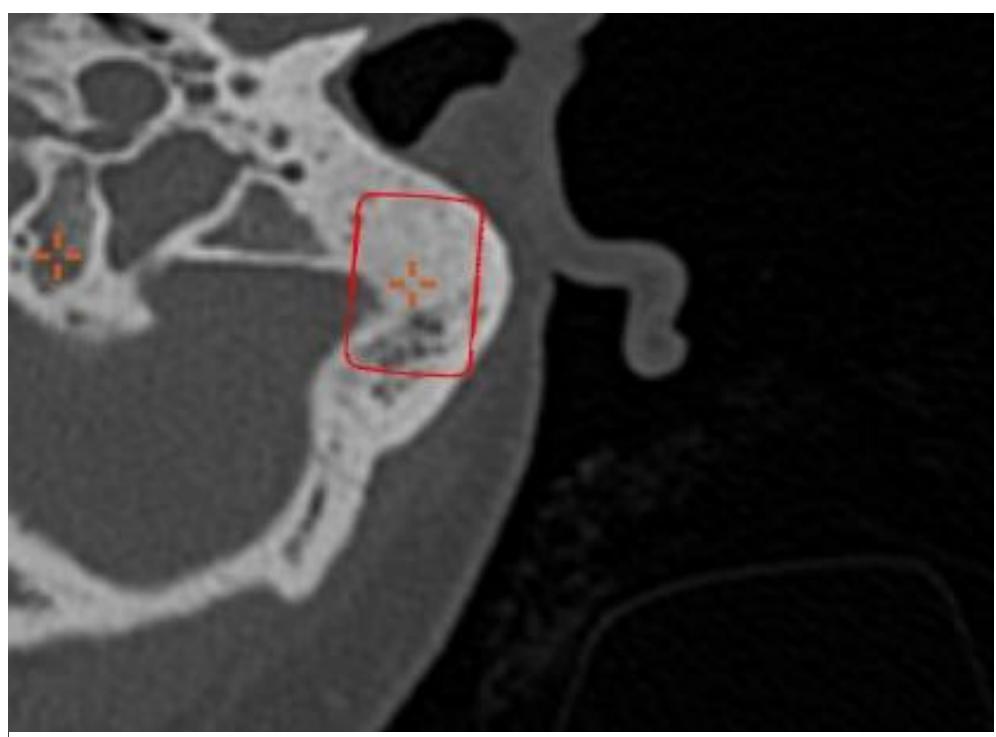

Abbildung 25: Kontakt zum Sinus sigmoideus. Die aufgelockerte Verschattung am rechten Bildrand zeigt das linke Mastoid des Patienten. In roter Umrandung ist die Schablone des Aktuators erkenntlich. Dieser reicht deutlich über das Mastoid nach intrakranial hinein, sodass von einer Impression des Sinus sigmoideus ausgegangen werden muss.

$\mathrm{Da}$ es sich im Mastoid und somit auch in dessen Residuum in der Regel um einen gut pneumatisierten Schädelknochen handelt, ist die sichere Schraubenfixierung des FMTs beurteilt worden: Besteht ein sicherer Sitz der Schrauben in voller Länge? Wie weit ist die maximal mögliche Eindringtiefe bei Schraube 1 bzw. bei Schraube 2? Wie stark ist die maximale Dicke der Knochenkompakta im Bereich der Schraube 1 bzw. der Schraube 2 (siehe Abbildung 26)? Hierbei wurde die nach bestmöglicher Implantation weiter ventral gelegene Schraube als Schraube 1 bezeichnet und die weiter dorsal gelegene Schraube als Schraube 2 definiert. 

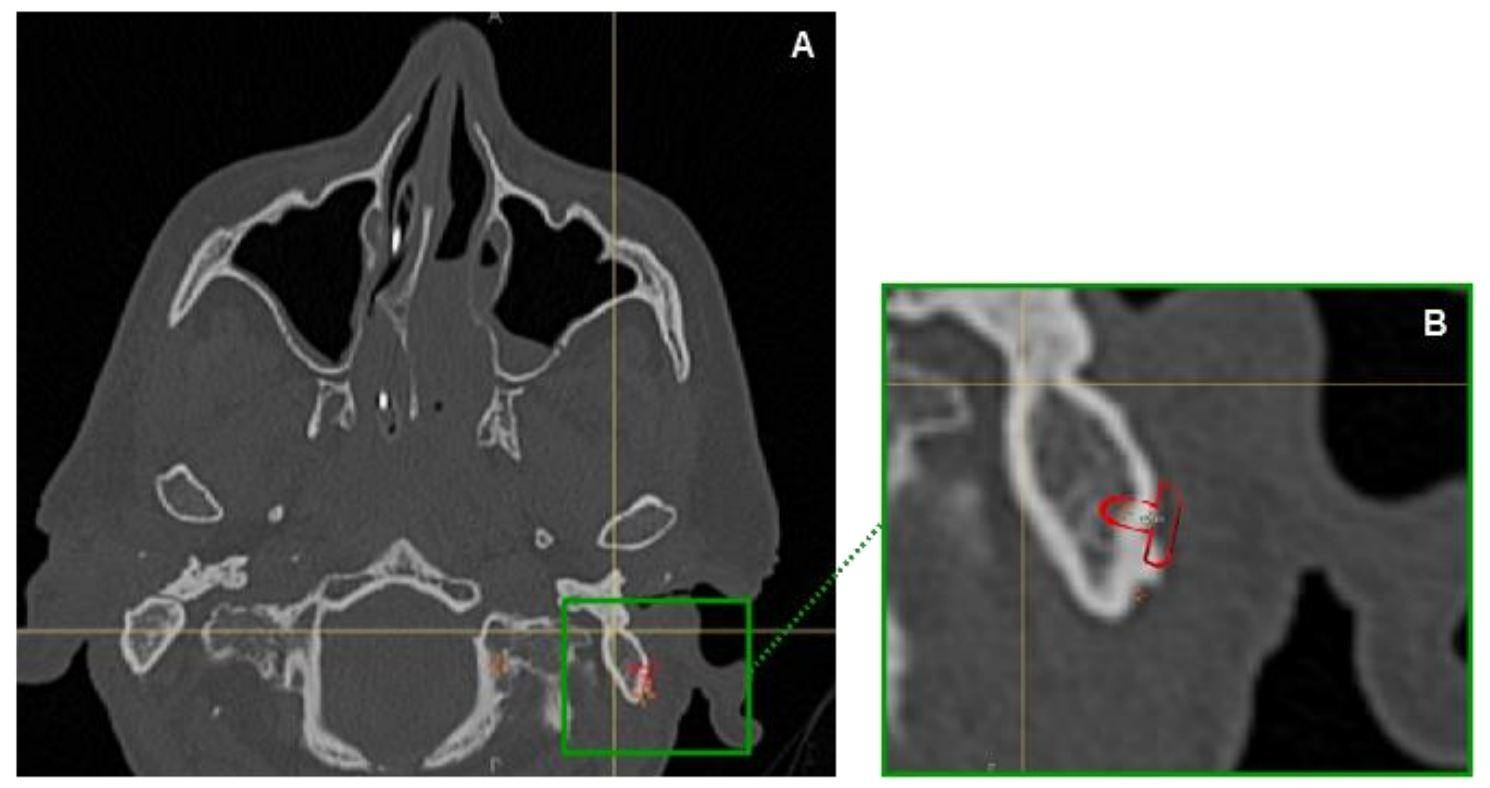

Abbildung 26: Maximale Dicke des nicht spongiösen Knochens bei Schraube 1. A Übersicht: Das craniale CT ist in axialer Bildgebung als Übersicht dargestellt. Die obere Bildkante entspricht der ventralen Patientenseite. Im Bereich des linken Mastoids (Ausschnitt) ist der der Schraube entsprechende Teil des templates in Rot erkennbar. B Ausschnitt: Die nicht spongiösen Anteile des Mastoidresiduums sind als knochendichte Verschattung abgebildet. Im Inneren ist der weichteildichtere, spongiöse Anteil. Im Bereich der Schraube 1 beträgt die maximale Dicke der Compacta 1,8 mm. 


\section{Ergebnisse}

Im Folgenden werden die Messergebnisse jeweils als Mittelwert über die 52 Patienten bzw. 60 Felsenbeine genannt. Zu Beginn werden die drei Lokalisationen einzeln betrachtet und anschließend im Kontext verglichen. Abschließend werden mögliche Geschlechterunterschiede bei den Messergebnisse dargestellt.

\subsection{Virtuelle Implantation in der retrosigmoidalen Lokalisation}

Die retrosigmoidale Lokalisation (siehe Abbildung 27) ist in der Literatur bereits als Alternative zum Mastoid für die Implantation des Masseschwingers des teilimplantierten, transkutanen Knochenleitungshörsystem Bonebridge beschrieben worden (Canis et al. 2013; Wimmer et al. 2015).

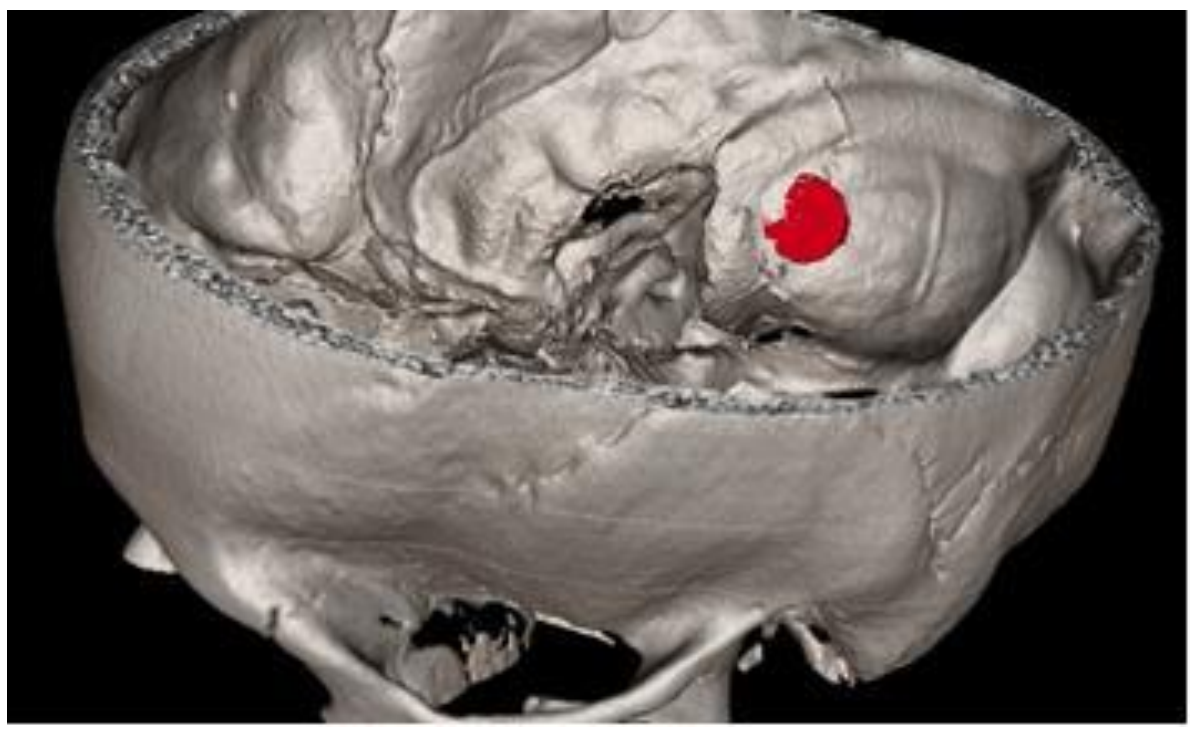

Abbildung 27: Virtuelle Implantation in der retrosigmoidalen Lokalisation. Es ist eine von der BBFastView Software generierte 3D-Rekonstruktion abgebildet. Der linke Bildrand entspricht der ventralen Schädelseite und es ist eine intrakranielle Ansicht dargestellt. In Rot ist die Basis des virtuell implantierten Masseschwingers zu sehen. Kranial davon ist der Verlauf des Sinus sigmoideus als Impression in die Schädelkalotte gezeigt.

Die Schädelkalotte ist im Durchschnitt im retrosigmoidalen Bereich mittig über dem virtuell implantierten Masseschwinger (BC-FMT) 6,4 2 mm dick. Die Werte reichen von einer 2,7 $\mathrm{mm}$ bis zu einer 11,6 $\mathrm{mm}$ dicken Schädelkalotte. Demnach wurde bei mehr als der Hälfte der Fälle (31 Patienten) eine Fläche der freiliegenden Implantatbasis Grad 3 bestimmt (vollständiges Freiliegen des BC-FMT). Bei weiteren 15 Patienten war mehr als die Hälfte der FMT-Basis nicht von Knochen bedeckt (Grad 2). Bei sechs der untersuchten 
Fälle war die Basis des FMT zu mehr als der Hälfte von Knochen bedeckt (Grad 1), in keinem Fall war bei virtueller Implantation in der retrosigmoidalen Lokalisation der FMT vollständig von Knochen bedeckt, sodass in allen Fällen ein Kontakt oder eine Impression der Dura die Folge wäre. Unterlegscheiben (BCI-lifts der Firma MED-EL) bieten die Möglichkeit, die Eindringtiefe des Implantats zu reduzieren und somit den Kontakt zur Dura zu verhindern. In sieben Fällen würden bereits $2 \mathrm{~mm}$ dicke Unterlegscheiben eine gewünschte Schonung der Dura erreichen. In weiteren zehn bzw. sechs Fällen könnte mit Unterlegscheiben von $3 \mathrm{~mm}$ bzw. $4 \mathrm{~mm}$ Stärke ohne Durakontakt implantiert werden. Dennoch wären es 29 Patienten (55,8\%), bei denen auch mit maximal dicken BCI-lifts von $4 \mathrm{~mm}$ der Kontakt zur Dura nicht vollständig verhindert werden kann. Im Durchschnitt käme es zu einer Duraimpression von $1,4 \pm 1 \mathrm{~mm}$.

Um die Verankerungsmöglichkeiten zu beurteilen, wurde die Stärke der knöchernen Schädeldecke bei den jeweiligen Schraubenösen gemessen. Im Durchschnitt liegt diese bei 7,3 $\pm 3 \mathrm{~mm}$, bei der ventralen Schraubenöse 9,2 $\mathrm{mm}$ und bei der dorsalen Schraubenöse 5,3 mm. Bei 39 Patienten (75\%) passen die Schrauben optimal und eine maximale Eindringtiefe von 3,9 $\mathrm{mm}$ in den Schädelknochen ist gegeben. Bei 14 Patienten ragt mindestens eine der Schrauben nach intrakranial über die Schädelkalotte hinaus, sodass von einer Beeinträchtigung der Dura ausgegangen werden kann. Durchschnittlich handelt es sich um $0,7 \mathrm{~mm}(0,2$ bis $1,6 \mathrm{~mm})$, um die in die Dura eingedrungen werden würde. In der Regel betrifft dies lediglich eine der zwei Schrauben.

Als weiterer wichtiger Aspekt gilt die Stärke der Kopfschwarte über der Spule, um eine möglichst verlustfreie Signalübertragung zwischen dem extrakranial getragenem Audioprozessor und Spule des implantierten Bonebridgeanteils zu gewährleisten. In 31 untersuchten voroperierten Mastoidregionen müsste das Epikranium über der Spule um 1 bis $2 \mathrm{~mm}$ ausgedünnt werden. Im Durchschnitt war die radiologisch sichtbare Kopfschwartendicke in diesem Bereich mit 8,4 \pm 3,5 mm dicker als die vom Hersteller empfohlenen 7 mm (Fa. MED-EL 2012). Eine Ausdünnung wird in diesen Fällen empfohlen.

Je weiter das BC-FMT von der Cochlea entfernt ist, desto höher ist der Schwingungsverlust (Eeg-Olofsson et al. 2008). Im Durchschnitt betrug die Distanz zwischen FMT und Cochlea in den radiologischen Untersuchungen bei retrosigmoidaler Platzierung 50,6 \pm 3,1 mm. Die Reichweiten erstreckten sich von 45,0 bis 59,1 mm.

Der Winkel des Aktuators zur Frankfurter Horizontalen war bei retrosigmoidaler Lage durchschnittliche $151 \pm 11,15^{\circ}$ groß. Zudem wurde die Dicke der radiologisch erkennbaren Kopfschwarte über dem Zentrum des FMTs gemessen, welche im Durchschnitt $11,8 \pm 4,9 \mathrm{~mm}$ betrug. 


\subsection{Virtuelle Implantation in der parietalen Lokalisation}

Die parietale Lokalisation, dorsokranial der Sutura parietomastoidea (siehe Abbildung 28), ist als Alternativlokalisation für das teilimplantierte transkutane Knochenleitungshörsystem noch aktueller Gegenstand der Forschung und wird in der Literatur in nur einer Kasuistik und einer Übersichtsarbeit als Implantationsalternative genannt (Canis et al. 2013; Zernotti und Sarasty 2015), wissenschaftliche Untersuchungen zur Eignung dieser Implantationslokalisation existieren nicht.

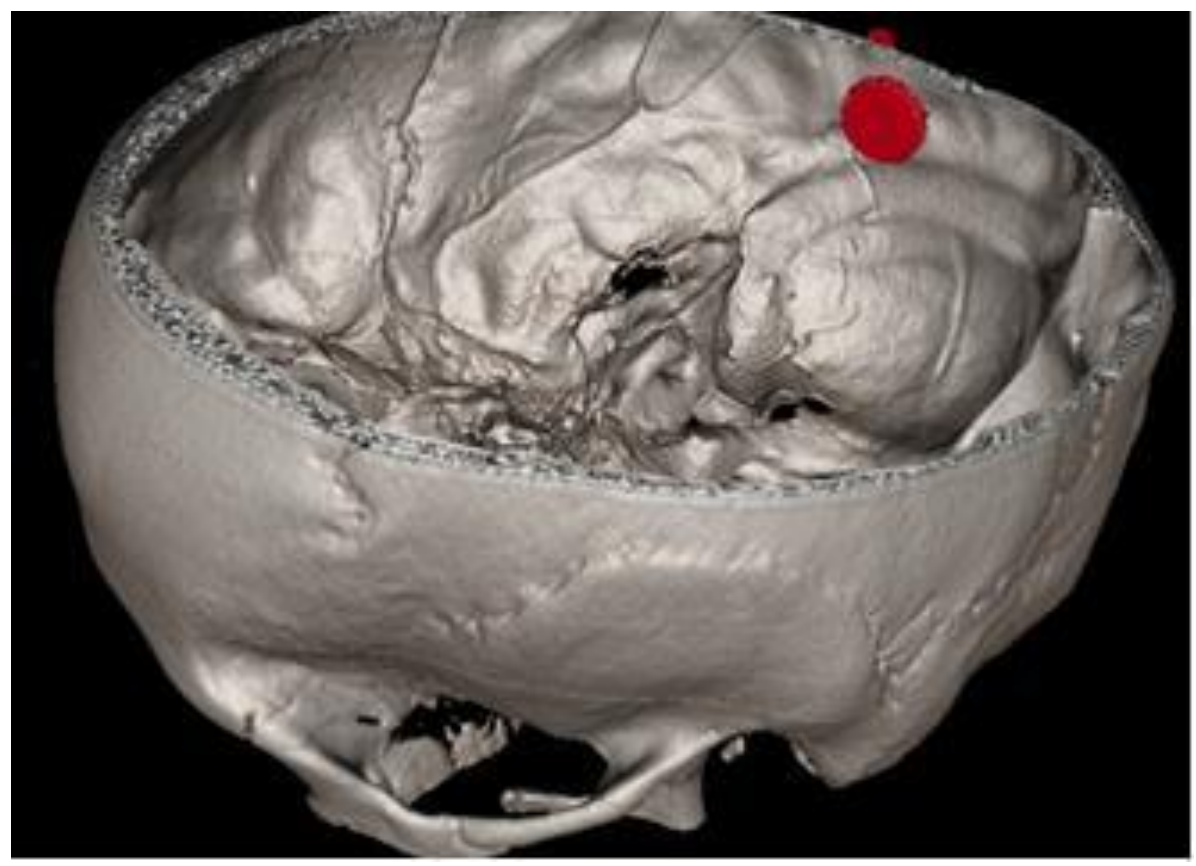

Abbildung 28: Virtuelle Implantation in der parietalen Lokalisation. Es ist eine 3D-Rekonstruktion der BBFastView-Software abgebildet. Der linke Bildrand entspricht der ventralen Schädelseite, und es ist eine intrakranielle Ansicht dargestellt. Als roter Kreis ist die Basis des virtuell implantierten Masseschwingers zu sehen. Nach kaudal ist der Verlauf der Sulcus sinus sigmoideus und transversus als Impression in die Schädelkalotte erkennbar.

Die Schädelkalotte ist im Durchschnitt im parietalen Bereich mittig über dem virtuell bestmöglich implantierten Masseschwinger 6,1 $\pm 1,7 \mathrm{~mm}$ dick. Die Werte reichen von 3,0 bis zu einer 9,9 mm dicken Schädelkalotte. Lediglich bei einem der untersuchten Fälle ist die Basis des Aktuators von intrakraniell gänzlich von Knochen bedeckt, sodass ein Kontakt zwischen BC-FMT und Dura ausgeschlossen wäre. Bei der Mehrheit (35 Patienten) kann die Basis bei bestmöglicher Implantation nicht von Schädelknochen bedeckt werden (Grad 3). Mehr als die Hälfte der Implantatbasis (Grad 2) hat bei elf Patienten und weniger als die Hälfte (Grad 1) bei fünf Patienten Kontakt zur Dura. Würden Abstandshalter verwendet, so könnte bei 55 \% der Fälle der Kontakt zwischen Dura und Basis des Implantats vermieden werden. Bei acht Patienten würden $2 \mathrm{~mm}$ bzw. in weiteren acht Fällen $3 \mathrm{~mm}$ Abstandshalter notwendig sein, um einen Kontakt zu vermeiden. In zwölf Fällen würde die 
vollständige Duraschonung durch $4 \mathrm{~mm}$ dicke Abstandshalter ermöglicht. Bei verbleibenden 23 Patienten (44,2 \%) ist eine vollständige Deckung der FMT-Basis mit Schädelknochen und somit ein ausbleibender Kontakt zur Dura auch mit maximalen Unterlegscheiben nicht möglich. Es verbleibt eine Impression von durchschnittlich $1,2 \pm 0,7 \mathrm{~mm}(0,3$ bis $3,0 \mathrm{~mm})$.

Die Stärke der knöchernen Schädeldecke bei den jeweiligen Schraubenösen wurde auf die gleiche Weise wie in der retrosigmoidalen Region gemessen. In 77 \% der Fälle können die Schrauben mit ihrer maximalen Eindringtiefe vollständig im Knochen fassen, da die Dicke der Kalotte im Bereich der parietal gelegenen Bohrlöcher im Schnitt 5,8 \pm 1,8 mm stark ist: bei der ventralen Schraubenöse durchschnittlich 5,1 $\mathrm{mm}$ und bei der dorsal gelegenen 6,6 mm. In Bereichen, in denen der Knochen dünner als die für die effektive Eindringtiefe idealen $4 \mathrm{~mm}$ ist (12 Fälle), kommt es durchschnittlich zu einem Hervortreten der Schraubenspitze nach intrakranial um $0,6 \mathrm{~mm}(0,1$ bis $1,1 \mathrm{~mm})$. In allen 52 virtuellen Implantationen sitzt mindestens eine Schraube mit vollständiger Eindringtiefe fest im Schädelknochen.

Die Dicke der Kopfschwarte im Bereich der Spule des implantierten Anteils der Bonebridge beträgt in der parietalen Implantationslokalisation im Durchschnitt 9,7 \pm 3,6 mm. Die Werte sind mit 2,2 bis zu 20,3 mm einer starken individuellen Schwankung unterlegen. Bei 78,4 \% (41 Patienten) müsste die Kopfschwarte auf 7 mm ausgedünnt werden.

Die Distanz zwischen BC-FMT und Cochlea in den radiologischen Untersuchungen bei parietaler Platzierung betrug im Durchschnitt 58,5 \pm 4,4 mm. Die Reichweiten erstreckten sich von 49,1 bis $68,2 \mathrm{~mm}$.

Der Winkel des Aktuatorzentrums zur Frankfurter Horizontalen betrug in parietaler Lage durchschnittliche 150,8 \pm 4,8 $8^{\circ}$. Die radiologisch messbare Kopfschwarte über dem Zentrum des FMTs war im Durchschnitt lediglich 7,81 \pm 3,09 $\mathrm{mm}$ dick und damit deutlich dünner als bei Implantation in retrosigmoidaler Position.

\subsection{Virtuelle Implantation im Bereich des Mastoidresiduums}

Bei der Implantation des BC-FMTs in das nach vorausgegangenen chirurgischen Eingriffen Residuum des Mastoids, wie beispielhaft in Abbildung 29 gezeigt, spielt die mögliche Freilegung des Aktuators in den pneumatisierten Räumen wie Gehörhang, Paukenhöhle und chirurgisch angelegter Mastoidhöhle eine besondere Rolle, da diese zum Erhalt von Entzündungsreaktionen beitragen könnte.

Im Bereich des Mastoidresiduums konnten 60 Fälle an 52 Patienten ausgewertet werden; 36 linksseitig und 24 rechtsseitig. Von diesen zeigten 32 eine vorangegangene canal-wall-upMastoidektomie und 28 eine canal-wall-down-Chirurgie. In 35 von 60 Fällen (58,3 \%) wurde die Implantation als möglich erachtet, da es zu keinem Freiliegen von Implantatteilen in den pneumatisierten Räumen wie Gehörgang, Paukenhöhle oder der chirurgisch angelegten 
Mastoidhöhle käme. Von diesen betrachteten 35 Mastoidknochen zählen 17 zu denen mit canal-wall-up-Mastoidchirurgie und 18 zu denen mit einer Radikalhöhle.

In 13,3 \% (8 Fälle) der bewerteten Mastoidresiduen ragte der Aktuator in pneumatisierte Räume vor, sowie in 20 \% (12) der Fälle die Schrauben des Implantats. In 14 (23,3 \%) ausgewerteten Mastoidresiduen bzw. virtuellen Implantationen des Aktuators wurde eine Impression des Sinus sigmoideus, trotz ausgewählten Abstandshaltern, von 0,3 bis zu 4,5 mm festgestellt.

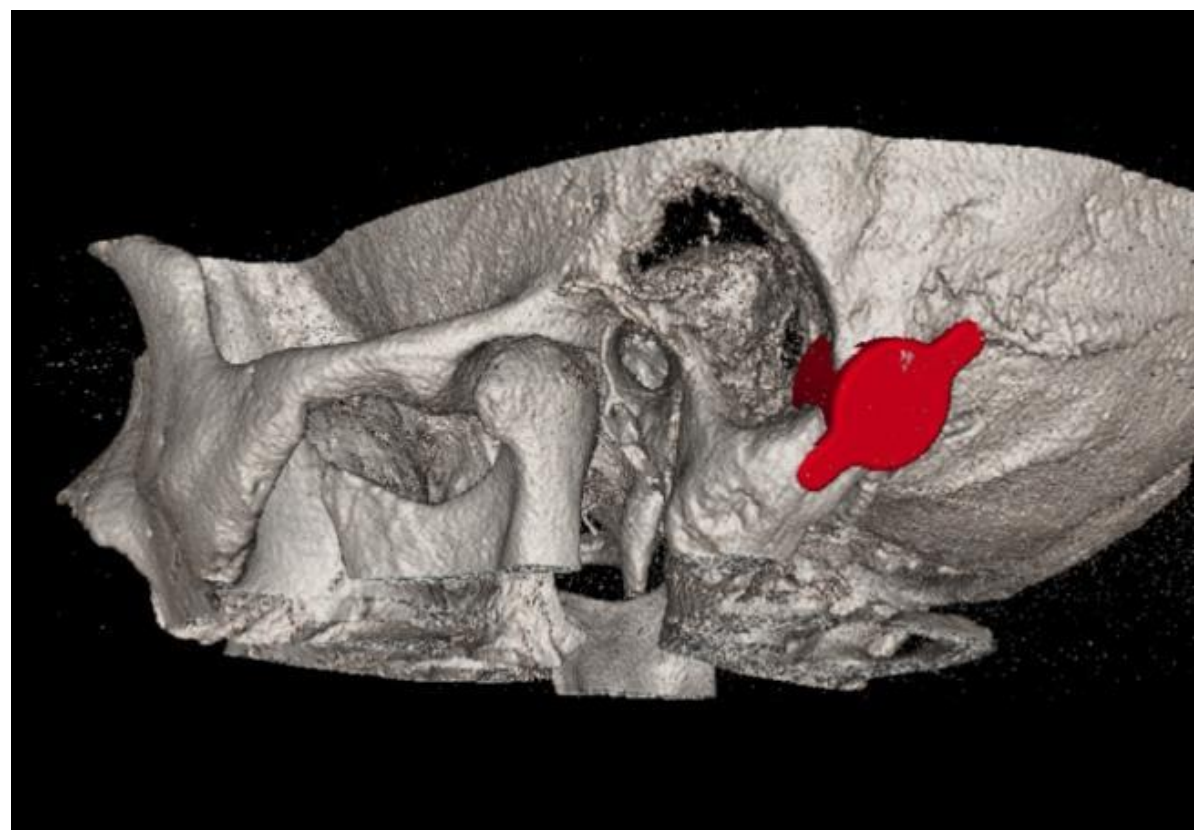

Abbildung 29: Virtuelle Implantation in das Mastoidresiduum. Es ist eine 3DRekonstruktion aus der BBFastView-Software abgebildet. Der linke Bildrand entspricht der ventralen Schädelseite, und das linke Kiefergelenk sowie der linke Gehörgang sind ersichtlich. In Rot ist das template des virtuell implantierten Masseschwingers zu sehen. Nach dorsokranial erstreckt sich zwischen Gehörgang und Aktuator die chirurgisch angelegte tympanomastoidale Höhle.

Von den 35 Mastoidresiduen konnte bei 8 Fällen eine virtuelle Implantation ohne Kontakt zu kritischen Strukturen wie die Sinus sigmoideus, Sinus transversus und Dura mater, durchgeführt werden. In 27 Fällen würde eine Anwendung von Abstandshaltern für eine sichere Implantation sorgen: in vier Fällen wären lediglich $1 \mathrm{~mm}$ Abstandshalter sinnvoll, bei weiteren vier Mastoiden wären $2 \mathrm{~mm}$ Unterlegscheiben notwendig und bei der Mehrheit (neun bzw. zehn Fällen) müssten Abstandshalter von 3 bzw. 4 mm Stärke eingebracht werden.

Die maximale Dicke der Kompakta im Bereich der zwei Schraubenösen wurde in den 35 Mastoiden, die für eine mögliche Implantation in Frage kommen würden, gemessen und variierte zwischen 0,7 und 12,4 mm. Das radiologisch ersichtliche und gemessene Epikranium über dem Bereich der Spule ergab Messwerte um die 7,7 \pm 2,9 $\mathrm{mm}$ (von 3,4 bis $16,1 \mathrm{~mm})$. Demnach müsste um nachfolgend den magnetisch gehaltenen Audioprozessor 
tragen zu können bei Implantation in 18 von 35 Fällen eine Ausdünnung der Kopfschwarte über der Spule durchgeführt werden.

Die Distanz zwischen BC-FMT Mittelpunkt und Cochlea in den radiologischen Untersuchungen bei Platzierung im Bereich des Mastoidresiduums betrug im Durchschnitt 41,2 \pm 3,1 mm. Die Reichweiten erstreckten sich von 33,8 bis 49,1 $\mathrm{mm}$.

Der Winkel zwischen Frankfurter Horizontaler und dem Zentrum des Masseschwingers betrug in dieser untersuchten Lokalisation im Durchschnitt 155,06 $\pm 10,6^{\circ}$.

\subsection{Vergleich der drei Implantationslokalisationen}

Die Implantation des Aktuators vom teilimplantierten Knochenleitungshörsystem in das Mastoidresiduum wird im Rahmen dieser Studie in lediglich 35 von 60 Fällen als möglich erachtet. Von diesen $35 \mathrm{zu}$ betrachtenden Mastoidresiduen waren acht ohne Kontakt zu Sinus sigmoideus, Sinus transversus oder Dura mater zu implantieren und weitere 27 mit Hilfe von Abstandshaltern. In 58,3 \% (35 von 60) der betrachteten Fälle könnte nach diesen Ergebnissen im Mastoidresiduum implantiert werden, ohne dass der Masseschwinger zu den pneumatisierten Strukturen frei zu liegen käme und ohne dass es zu Kontakt zu Sinus sigmoideus, Sinus transversus oder Dura mater käme.

In den Alternativlokalisationen retrosigmoidal und parietal wurden 52 Patienten ausgewertet. Nach den hier durchgeführten Untersuchungen kann in 44,2 \% der Fälle (23 von 52) mit Hilfe von Unterlegscheiben ohne Kontakt zu Dura oder den Sinus in der retrosigmoidalen Lokalisation implantiert werden. Im Bereich dorsokranial der Sutura parietomastoidea (parietal) sind es 55,8\% (29 von 52) der Patienten bei denen eine Implantation ohne Duraimpression oder Kontakt zu den venösen Sinus möglich erscheint, dies ebenfalls mit Hilfe von Abstandshaltern.

Es entsteht im Vergleich bezüglich Duraimpression und Sinusschonung folgende Rangfolge: Am häufigsten scheint, auch nach chirurgischen Eingriffen, das Mastoid mit 58,3\% geeignet zu sein. Mit 55,8\% weicht die parietale Region jedoch nur geringfügig von der Implantationsmöglichkeit im Mastoidresiduum ab. Die retrosigmoidale Region, die in der Literatur als Alternative zum unversehrten Mastoid bereits vorgeschlagen wird (Barbara et al. 2013; Canis et al. 2013; Wimmer et al. 2015), schneidet mit 44,2 \% im Rahmen dieser Studie als am wenigsten häufig für eine Implantation geeignet ab.

Betrachtet man die retrosigmoidale und parietale Lokalisation an einem Patienten, zeigte sich, dass in 69,2\% (36 von 52) der Kontakt zur Dura mater und zu den venösen Sinus sich wenigstens in einer der beiden alternativen Implantationsstellen vermeiden lässt. Bei den verbleibenden 30,8 \% (16) muss auch in der bestausgewählten Position eine durchschnittliche Duraimpression von $1,0 \pm 0,5 \mathrm{~mm}(0,3$ bis $2,4 \mathrm{~mm})$ in Kauf genommen werden. Bei der Mehrheit der betrachteten Fälle (55,8 \% / 29 Patienten) konnte die Duraimpression im parietalen Bereich als geringer gemessen werden als in der im Vergleich stehenden retro- 
sigmoidalen Alternativlokalisation. Die Wahl der parietalen Lokalisation führt in 29 Fällen zu einer Reduktion der Duraimpression von 5,0 \pm 1,5 $\mathrm{mm}$ an der retrosigmoidalen Position verglichen mit 3,4 $\pm 1,4 \mathrm{~mm}$ an der parietalen. Lediglich bei einem Patienten (1,9\%) war in der Tiefe der Duraimpression zwischen retrosigmoidaler und parietaler Position kein Unterschied festzustellen. Insgesamt muss jedoch darauf hingewiesen werden, dass keine signifikanten Unterschiede in der durchschnittlichen Dicke der Schädelkalotte und der damit zusammenhängenden durchschnittlich notwendigen Tiefe der Duraimpression bei den zwei Alternativlokalisationen gefunden werden konnten.

Bezüglich des chirurgischen Mehraufwands für die oftmals notwendige Ausdünnung des Epikraniums über dem Bereich der Spule bzw. des magnetisch gehaltenen externen Audioprozessors haben sich für die drei Implantationsregionen folgende Daten ermitteln lassen: Im restmastoidalen Bereich liegt die radiologisch ersichtliche, durchschnittliche Dicke der Kopfschwarte mit 7,7 \pm 2,9 $\mathrm{mm}$ (von 3,4 bis 16,1 $\mathrm{mm}$ ) lediglich geringfügig über den vom Hersteller empfohlenen $7 \mathrm{~mm}$ (Fa. MED-EL 2012). Dennoch muss in mehr als der Hälfte $(51,4 \%)$ der Patienten eine Ausdünnung vorgenommen werden, um eine einwandfreie Signalübertragung gewährleisten zu können. In der retrosigmoidalen Position müsste bei 59,6\% der Patienten eine Kopfschwartenausdünnung erfolgen. Die Messwerte liegen im Durchschnitt bei 8,4 \pm 3,5 mm (von 2,6 bis 19,3 mm). Bei einer Platzierung des Implantats dorsokranial der Sutura parietomastoidea weichen die durchschnittlichen Messwerte des radiologisch ersichtlichen Epikraniums mit 9,7 \pm 3,6 mm (von 2,2 bis 20,3 mm) bereits weiter von den vom Hersteller empfohlenen $7 \mathrm{~mm}$ ab. Mehr als $78 \%$ der Patienten müssten bei Implantation im parietalen Bereich eine Ausdünnung der Kopfschwarte vornehmen lassen.

Bei der durchschnittlichen Distanz des Masseschwingers zur Cochlea , ist in den drei unterschiedlichen Positionen eine deutliche Rangfolge ersichtlich: Die Platzierung im Mastoidresiduum hat mit durchschnittlich 41,2 \pm 3,1 $\mathrm{mm}(33,8$ bis 49,1 $\mathrm{mm})$ den kürzesten Abstand zur Cochlea. Hier sind 35 Messungen durchgeführt worden, da in weiteren Patientenfällen, die vorangegangene chirurgische Intervention kein Residuum des Mastoids ergeben hat und somit dieser Bereich nicht mehr existierte. Eine Implantation im Mastoidresiduum wäre folglich nicht in jedem Fall zu empfehlen. Im mittleren Bereich liegt die retrosigmoidale Lokalisation, bei welcher eine durchschnittliche Strecke von 50,6 \pm 3,1 mm (45 bis $59,1 \mathrm{~mm}$ ) zwischen Zentrum des Aktuators und Cochlea gemessen werden konnte. Eine Platzierung des BC-FMTs in der parietalen Region führt mit durchschnittlichen Messwerten von 58,5 \pm 4,4 $\mathrm{mm}$ zu einer relativ großen Distanz zwischen Implantat und Cochlea. Es handelt sich bei dem gezeigten Vergleich um statistisch signifikante Unterschiede. Eine Übersicht der Messungen zeigt Tabelle 4 in 6.1 .

Der Winkel zwischen Frankfurter Horizontaler und dem Aktuator liegt sowohl bei retrosigmoidaler als auch parietaler Implantation bei ca. 150,9 ${ }^{\circ}$. Bei Implantation ins Mastoidresiduum beträgt der Winkel im Durchschnitt $5^{\circ}$ mehr $\left(155,06^{\circ}\right)$. 
Die Werte der Dicke der Kopfschwarte über dem FMT wichen um $\pm 3 \mathrm{~mm}$ sowohl retrosigmoidal als auch parietal von den Durchschnittswerten der Dicke über der Spule ab.

\subsection{Geschlechtsspezifische Unterschiede}

In dieser Studie wurden radiomorphologisch Felsenbeine von 25 männlichen und 27 weiblichen Fällen untersucht.

Die Distanz zwischen BC-FMT Zentrum zur Cochlea war bei männlichen Patienten im Schnitt größer als bei weiblichen; dies insbesondere in retrosigmoidaler $(\mathrm{P}=0,01)$ und restmastoidaler ( $\mathrm{P}=0,03)$ Lokalisation (siehe 6.1 Tabelle 1 und Tabelle 3). In der parietalen Lokalisation konnte diesbezüglich kein geschlechtsspezifischer Unterschied festgestellt werden (siehe 6.1 Tabelle 2).

Bezüglich der gemessenen Knochenstärke und der damit in Zusammenhang stehenden durchschnittlichen Duraimpression konnten weder in retrosigmoidaler noch parietaler Position Unterschiede zwischen den Geschlechtern festgestellt werden.

Das radiologisch ersichtliche und mittels der BBFastView Software gemessene Epikranium im Bereich der Spule, war bei männlichen Probanden im Schnitt etwas dicker, wenn im Bereich des Mastoids implantiert wurde $(\mathrm{P}<0,01$; siehe 6.1 Tabelle 3).

Eine tabellarische Übersicht der nach Messung und Auswertung erhaltenen Daten ist in 6.1 zu finden. 


\section{Diskussion}

Diese Studie ist die erste, die drei Implantationslokalisationen für den Aktuator eines transkutanen teilimplantierten aktiven Knochenleitungshörsystems miteinander vergleicht. In einer Übersichtsarbeit sind bereits Angaben zu den erschwerten Implantationsbedingungen nach chirurgischen Eingriffen am Mastoid und Mittelohr beschrieben (Zernotti und Sarasty 2015). In der Regel wird die retrosigmoidale Region als Alternative vorgeschlagen (Canis et al. 2013, Barbara et al. 2013 und Law et al. 2016). Die Studie von Law et al. 2016 mit 16 Patienten beschreibt drei Fälle, in denen auf Grund von vorausgegangener mastoidaler Chirurgie auch die retrosigmoidale Alternativlokalisation keine Lösung bietet. Dies verdeutlicht, dass weitere Alternativlokalisationen wie die hier untersuchte Position dorsokranial der Sutura parietomastoidea (parietal) durchaus von Relevanz sind. Nach den im Rahmen dieser Studie durchgeführten Bewertungskriterien ist die parietale Lokalisation vergleichsweise besser für die Aufnahme der FMTs geeignet als die in der Literatur beschriebene Alternative retrosigmoidal. In der letztgenannten Lokalisation können 23 von 52 Patienten und parietal 29 von 52 Patienten implantiert werden, ohne dass es zur Impression von Dura oder Sinus kommen muss. In beiden Fällen ist die Nutzung von Abstandshaltern berücksichtigt.

Obwohl die parietale Lokalisation in Bezug auf Distanz zwischen dem Masseschwinger und der Cochlea am schlechtesten abschneidet, handelt es sich unter Berücksichtigung aller anderen erhobenen Parameter um eine vielversprechende Alternativlokalisation. Es wurde gezeigt, dass eine lange Strecke zwischen Aktuator und Cochlea mit einem entsprechenden Energieverlust einhergeht, sodass eine Minderung des Hörergebnisses denkbar ist (EegOlofsson et al. 2008; Eeg-Olofsson et al. 2013). Dennoch beschreiben Law et al., dass es keine signifikanten Unterschiede im Hörgewinn zwischen den Versuchsgruppen transmastoidaler und retrosigmoidaler Implantationen gegeben habe (Law et al. 2016), obwohl die Cochlea nach unserer Studie zur retrosigmoidalen Platzierung um durchschnittliche $9,4 \mathrm{~mm}^{4}$ weiter entfernt liegt als die Cochlea zur FMT-Platzierung im Restmastoid. Aktueller Gegenstand der Forschung ist in diesem Sinne auch der mögliche Einfluss von Weichgewebe und Körperflüssigkeiten wie Blut oder Liquor bei der Schallleitung (Ito et al. 2011; Perez et al. 2011; Stenfelt 2011).

Während der radiologisch-anatomischen Untersuchung der Schädel, wurde deutlich, dass die Mehrheit an Implantationen nur auf Grund der Nutzung von Abstandshaltern (BCIlifts), ohne Kontakt und damit möglicher Beeinträchtigung von anatomischen Strukturen wie Sinus sigmoideus, Sinus transversus oder Dura mater, möglich sein würde. Dies ist ebenso Ergebnis aus der aktuellen Studie von Law et al. mit jedoch lediglich 16 untersuchten Fäl-

\footnotetext{
4 50,6 mm (Ø-Distanz Cochlea/ retrosigmoidal) - 41,2 mm (Ø-Distanz Cochlea/ Restmastoid $)=9,4 \mathrm{~mm}$
} 
len (Law et al. 2016). Beispielsweise konnte im Rahmen der vorliegenden Untersuchung im retrosigmoidalen Bereich eine durchschnittliche Schädeldicke mittig über dem FMT von 6,4 $\pm 2 \mathrm{~mm}$ gemessen werden, was im Einklang mit den Ergebnissen von Lang und Samii steht, die ebenfalls 6,4 mm Dicke für die Schädelkalotte im retrosigmoidalen Bereich angeben (Lang und Samii 1991). Auch Law et al. kommen in diesem Bereich bei ihren Patienten auf ähnliche Werte mit durchschnittlichen $6,7 \mathrm{~mm}$. Alle drei Messungsergebnisse zeigen demnach eine deutliche Abweichung von der Dicke der Schädelkalotte und der notwendigen Implantationstiefe, um den Aktuator mit 8,7 mm Tiefe ohne Kontakt oder Impression zur Dura einbringen zu können. Zudem spricht auch die relativ große Varianz zwischen den Messwerten bei unterschiedlichen Patienten für die Nutzung einer präoperativen, individuellen Computertomographie-gestützten Planung, wie es mit der im Rahmen der vorliegenden Studie verwendeten BBFastView-Software denkbar wäre. Eine CT-gestützte präoperative Planung ist einheitlich in der Literatur empfohlen (Canis et al. 2013; Huber et al. 2013; Plontke et al. 2014; Matsumoto et al. 2015; Zernotti und Sarasty 2015).

Es werden neben der BBFastView Planungssoftware noch weitere mögliche Programme in der Literatur erwähnt, die ähnlichem Zweck dienen sollen: Der 3D Slicer (3D Slicer 2017) wurde anfangs auch im Rahmen dieser Promotionsarbeit getestet. Es handelt sich hierbei ebenfalls um eine frei zugängliche Software zur Analyse und Visualisierung medizinischer Bilder. Es besteht die Möglichkeit, einzelne Bereiche wie beispielsweise das Felsenbein als 3D-Visualisierung auszuschneiden und sich für die präoperative Planung zu Nutze zu machen (http://www.slicer.org). Canis et al. haben diese Software genutzt, um in einer Kasuistik über zwei Patienten die präoperative radiographische Planung für eine FMTImplantation im retrosigmoidalen Bereich durchzuführen (Canis et al. 2013). Im Vergleich zur hier genutzten BBFastView-Software schien der 3D Slicer dennoch deutlich aufwendiger in der Handhabung. Während bei BBFastView nach dem Laden der DICOMDatensätze direkt intuitiv mit der virtuellen Implantation begonnen werden kann, ist die vorherige Erstellung eines ausgeschnittenen Schädelbereichs als 3D-Modell deutlich zeitintensiver. Weiter stellen Todt et al. einen Vibrant-Bonebridge-Viewer vor, der wichtige anatomische Strukturen (Sinus sigmoideus, Dura, äußerer Gehörgang, Labyrinth, Kortikalis) selbstständig aus den CT-DICOM-Datensätzen erkennt und ein dem Patienten entsprechend individuelles 3D-Modell des Felsenbeins generiert (Todt et al. 2014). Mittels farblicher Codierung wird nicht nur die Dicke der Schädelkalotte angezeigt, sondern beispielsweise auch die Kompression von Sinus und Meningen in der Signalfarbe Rot dargestellt. Ist der Abstand zu diesen Strukturen $<1 \mathrm{~mm}$, aber besteht noch keine Impression der Struktur, so erscheint die Farbe Gelb. Als weiterer Vorteil gegenüber der BBFastView-Software kann hier das Einbringen der mehrheitlich notwendigen Abstandshalter simuliert werden (Todt et al. 2014). Zudem ist auch neben dem generierten 3D-Modell eine Ansicht in sagittaler, axialer und koronarer Schichtung (Todt et al. 2014) wie auch in der Software BBFastView möglich. Bei der in der vorliegenden Studie verwendeten Software bewirkte die Aktivierung des 3D-Views eine erhebliche Verzögerung im Bildablauf, sodass die 3D-Ansicht lediglich 
als Kontrolle genutzt wurde und im axialen (nicht rekonstruierten) Fenster die Messungen durchgeführt wurden. Ähnliche Einschränkungen wären dementsprechend auch bei dem vorgestellten Vibrant-Bonebridge-Viewer zu erwarten. Zudem erläutern Todt et al. lediglich die Nutzung für eine mastoidale Implantationsplanung (Todt et al. 2014), sodass die Frage verbleibt, ob das Programm für eine Auswertung von Alternativlokalisationen ebenso anwendbar wäre.

Eine weitere Publikation beschreibt eine topographische Karte der Knochendicke am Schädel, die so genannte TBTM (topographic bone thickness map), bei der ebenfalls mittels Farbcodierung die Schädeldicke abgelesen werden kann (Wimmer et al. 2015). Aber auch hier wird lediglich die präoperative Planung für die Standardlokalisation des Mastoids beschrieben (Wimmer et al. 2015). Insgesamt scheint nicht nur Einigkeit über den Wert der radiographischen präoperativen Planung mittels Software zu bestehen, sondern es wird diskutiert, dass die Frage nach einer sinnvollen Umsetzung zwischen der präoperativen Planung mit der tatsächlichen, chirurgischen Situation noch unbeantwortet ist (Güldner et al. 2013; Matsumoto et al. 2015). Canis et al. berichten 2013 von einer möglichen navigationsgestützten Identifikation des Sinus sigmoideus im Rahmen einer retrosigmoidalen Implantation; weiter wird aber auch die Nutzung extrakranialer Landmarken genannt, wie die Spina suprameatum und die Protuberantia occipitalis externa, die aber lediglich orientierend weiterhelfen könnten (Canis et al. 2013; Plontke et al. 2014). Weiter beschreibt eine japanische Forschergruppe die Implantation ins Mastoid bei drei Patienten mit Hilfe eines entwickelten „surface template-assisted marker positioning (STAMP)“-Systems, welches insbesondere zum Einsatz kommen könnte, wenn bereits präoperativ ersichtlich wird, dass die Anlage der zylindrischen Knochennische mit der Freilegung von Sinus und/oder Meningen einhergehen wird (Matsumoto et al. 2015).

Unsere Ergebnisse zeigen - aber auch Barbara et al. berichten - dass es vermutlich immer Patientenfälle geben wird, bei denen eine Implantation mit Sinus- und/oder Duraimpression einhergehen wird (Barbara et al. 2013). Bei 30,8 \% der von uns untersuchten Fälle verbleibt, unabhängig von der Lokalisation, auch in der am besten ausgewählten Position, eine durchschnittliche Duraimpression von $1,0 \pm 0,5 \mathrm{~mm}(0,3$ bis 2,4 mm). Einige Chirurgen schreiben, dass eine geringfügige Kompression von Sinus und Dura durchaus zu akzeptieren wäre und Komplikationen nicht bekannt seien (Zernotti und Sarasty 2015). Dennoch besteht Einigkeit, dass dieses nach Möglichkeit zu vermeiden ist, da bisher keine Langzeitstudien, die ein mögliches Risiko ausschließen, existieren. Kürzlich wurde jedoch ein Patientenfall beschrieben, bei welchem im Rahmen einer BAHA-Implantation ein subdurales Hämatom als schwerwiegende Komplikation aufgetreten sei (Amin und Aymat-Torrente 2017). Ähnliche Fälle sind dementsprechend auch bei der Implantation für die Bonebridge denkbar. 
Ein kleinerer Aktuator mit einer Tiefenreduktion um 2,3 $\mathrm{mm}^{5}$ würde die bisherigen chirurgischen Limitationen im retrosigmoidalen Bereich erheblich verringern und würde sich auch im Mastoidresiduum und in der parietalen Region zusätzlich positiv auswirken. Auch andere Arbeitsgruppen sehen eine abschließende Problemlösung vor allem in der Größenreduktion des Masseschwingers (Håkansson et al. 2008; Plontke et al. 2014; Law et al. 2016; Rahne et al. 2016). Bis dahin könnte aber die u. a. in dieser Studie untersuchte parietale Lokalisation eine weitere, vielversprechende Option bei der Implantation des teilimplantierten aktiven Knochenleitungshörsystems sein.

Die Messungen dieser Studie sind folgenden Limitationen unterlegen. Um möglichst genaue Daten zu erzielen, wurden lediglich Feinschicht Computertomographien mit 0,6 mm Schichtdicke in die Studie einbezogen, wie es in einer japanischen Untersuchung zur „Template-guided implantation of the Bonebridge: clinical experience" (Matsumoto et al. 2015) ebenso gehandhabt wurde. Computertomographien von Patienten mit bereits vorhandenen Implantaten wurden von der Studie ausgeschlossen, da diese oft zu Artefakten und Verzerrungen im CT führen können. Weiter sind alle Messungen von einer Person durchgeführt worden, um interindividuelle Unterschiede zwischen verschiedenen Untersuchern auszuschließen. Die Position und Ausdehnung der Sinus wurde mit Hilfe der Schädelimpressionen als Landmarken bestimmt. Denkbar wäre, dass kontrastmittelgestützte CTs die Analyse hätten unterstützen können. Weiter wird die Messung der Dicke des radiologisch ersichtlichen Epikraniums durch die Kopfposition und Kopfauflage im CT-Scanner beeinflusst, sodass diese Messwerte besonders zu hinterfragen sind. Insbesondere bei Patienten mit erheblichen Anteilen von Unterhautfettgewebe, ist die Verformung der Kopfschwarte im CTScanner wahrscheinlich.

Mit den Messungen des Winkels zwischen Frankfurter Horizontaler und dem Zentrum des implantierten Masseschwingers konnten im Rahmen dieser Arbeit auf keine neuen Erkenntnisse geschlossen werden. Die Frankfurter Horizontale wird als Landmarke für den horizontalen Verlauf des zu schonenden Sinus sigmoideus beschrieben (Ucerler und Govsa 2006), sodass unsere Ergebnisse eventuell im Rahmen zukünftiger Arbeiten hilfreich sein könnten.

Aus dieser Arbeit ergeben sich weitere wichtige Forschungsaspekte. Nach der hier beschriebenen virtuellen Implantation wären beispielsweise klinische Studien über die tatsächliche parietale Implantationslokalisation denkbar. In diesem Zusammenhang könnten audiologische Untersuchungen interessant sein, die eventuell weiter Aufschluss geben würden, inwieweit die relativ große Distanz zur Cochlea (parietal durchschnittlich 58,5 mm) einen Einfluss auf die Hörschwelle nehmen würde. Hier sei jedoch gesagt, dass eine postoperative Abmessung des implantierten Masseschwingers an radiologischen Untersuchungen auf Grund der zu erwartenden Artefakte nicht möglich wäre. Folglich müsste eine

\footnotetext{
5 8,7 mm (Tiefe des FMT) - 6,4 mm (Ø Schädeldicke im retrosigmoidalem Bereich) $=2,3 \mathrm{~mm}$
} 
präoperativ bestimmte Implantationslokalisation nach tatsächlicher Implantation in diesem Bereich mit dem postoperativen Hörergebnis korreliert werden. 


\section{$5 \quad$ Zusammenfassung}

Der Vergleich der drei Implantationslokalisationen restrosigmoidal, parietal und mastoidal mit Hilfe einer CT-gestützten virtuellen Implantation, ist besonders bei Patienten mit vorangegangener Mastoidchirurgie, sinnvoll. Die Region dorsokranial der Sutura parietomastoidea (parietale Implantationslokalisation) scheint nach den Ergebnissen dieser Studie eine vielversprechende Alternative neben den anderen Lokalisationen zu sein, die in Zukunft von Chirurgen neben der bereits häufig gewählten retrosigmoidalen Position in Betracht gezogen werden könnte. Es hat sich ebenso bestätigt, dass eine individuelle, softwaregestützte präoperative Planung vor jeder Implantation von aktiven Knochenleitungshörsystemen zu empfehlen ist. Die Lage zu anatomischen Strukturen ist auf diese Weise besser einzuschätzen, und Impressionen von Dura und den venösen Sinus sollen möglichst vermieden werden. Weitere Studien, die den Einfluss verschiedener Implantationslokalisationen und damit auch unterschiedliche Entfernungen zur Cochlea auf den Hörerfolg untersuchen, könnten sich dieser Arbeit anschließen. Weiter sind Umsetzungsmöglichkeiten der präoperativen Planung in die chirurgische Situation am Patienten noch zu konkretisieren. 


\section{Anhang}

\subsection{Tabellen}

Tabelle 1: Messergebnisse und geschlechtsspezifische Unterschiede nach Implantation in die retrosigmoidale Lokalisation. Die Messwerte sind in Millimeter angegeben. Signifikante p-Werte sind fett hervorgehoben. Die maximal angegebene Duraimpression bezieht sich auf Implantationen mit Abstandshaltern (BCI-lifts) *.

\begin{tabular}{|c|c|c|c|c|c|c|c|c|c|c|c|c|}
\hline & \multicolumn{3}{|c|}{\begin{tabular}{|l} 
Dicke der \\
Schädelkalotte
\end{tabular}} & \multicolumn{3}{|c|}{$\begin{array}{l}\text { max. Impression } \\
\text { der Dura* }\end{array}$} & \multicolumn{3}{|c|}{$\begin{array}{l}\text { Abstand zur } \\
\text { Cochlea }\end{array}$} & \multicolumn{3}{|c|}{\begin{tabular}{|l} 
Dicke des \\
Epikraniums über der \\
Spule
\end{tabular}} \\
\hline & $\varnothing$ & SA & Fälle & $\varnothing$ & SA & Fälle & $\varnothing$ & SA & Fälle & $\varnothing$ & SA & Fälle \\
\hline क und $\hat{\delta}$ & 6,4 & 2,0 & 52 & 1,4 & 1,0 & 29 & 50,6 & 3,1 & 52 & 8,4 & 3,5 & 51 \\
\hline $0^{\lambda}$ & 6,0 & 2,3 & 25 & 1,7 & 0,9 & 13 & 51,7 & 3,2 & 25 & 9,3 & 3,9 & 25 \\
\hline 우 & 6,7 & 1,8 & 27 & 1,1 & 1,0 & 16 & 49,5 & 2,7 & 27 & 7,6 & 3,1 & 26 \\
\hline $\begin{array}{l}o \text { im Vergleich } \\
\text { mit } q \text { (P-Wert) }\end{array}$ & 0,25 & & & 0,10 & & & 0,01 & & & 0,09 & & \\
\hline
\end{tabular}

Tabelle 2: Messergebnisse und geschlechtsspezifische Unterschiede nach Implantation in der parietalen Lokalisation. Die folgenden Messwerte sind in Millimeter angegeben. Die maximal angegebene Duraimpression bezieht sich auf Implantationen mit Abstandshaltern (BCI-lifts) *. Für die untersuchten Parameter Dicke der Schädelkalotte, max. Duraimpression, Abstand zur Cochlea und Dicke des Epikraniums über der Spule, wurden in der parietalen Region keine signifikanten Unterschiede berechnet.

\begin{tabular}{|c|c|c|c|c|c|c|c|c|c|c|c|c|}
\hline & \multicolumn{3}{|c|}{\begin{tabular}{|l} 
Dicke der \\
Schädelkalotte
\end{tabular}} & \multicolumn{3}{|c|}{$\begin{array}{l}\text { max. Impression } \\
\text { der Dura* }\end{array}$} & \multicolumn{3}{|c|}{$\begin{array}{l}\text { Abstand zur } \\
\text { Cochlea }\end{array}$} & \multicolumn{3}{|c|}{$\begin{array}{l}\text { Dicke des } \\
\text { Epikraniums über der } \\
\text { Spule }\end{array}$} \\
\hline & $\varnothing$ & SA & Fälle & $\varnothing$ & SA & Fälle & $\varnothing$ & SA & Fälle & $\varnothing$ & SA & Fälle \\
\hline$q$ und $\hat{o}$ & 6,1 & 1,7 & 52 & 1,2 & 0,7 & 23 & 58,8 & 4,4 & 52 & 9,7 & 3,6 & 51 \\
\hline o & 6,2 & 1,6 & 25 & 1,1 & 0,8 & 11 & 59,1 & 3,9 & 25 & 10,5 & 3,7 & 25 \\
\hline o & 6 & 1,7 & 27 & 1,2 & 0,7 & 12 & 58,5 & 5 & 27 & 9 & 3,5 & 26 \\
\hline $\begin{array}{l}0 \text { im Vergleich } \\
\text { mit } q \text { (P-Wert) }\end{array}$ & 0,62 & & & 0,67 & & & 0,62 & & & 0,13 & & \\
\hline
\end{tabular}


Tabelle 3: Messergebnisse und geschlechtsspezifische Unterschiede nach Implantation in das Mastoidresiduum. Die folgenden Messwerte sind in Millimeter angegeben. Signifikante P-Werte sind fett hervorgehoben. Die maximale angegebene Duraimpression bezieht sich auf Implantationen mit Abstandshaltern (BCI-lifts) *.

\begin{tabular}{|c|c|c|c|c|c|c|}
\hline & \multicolumn{3}{|c|}{$\begin{array}{l}\text { Abstand zur } \\
\text { Cochlea }\end{array}$} & \multicolumn{3}{|c|}{$\begin{array}{l}\text { Dicke des } \\
\text { Epikraniums über der } \\
\text { Spule }\end{array}$} \\
\hline & $\varnothing$ & SA & Fälle & $\varnothing$ & SA & Fälle \\
\hline$q$ und $\hat{\alpha}$ & 41,4 & 3 & 40 & 7,8 & 2,8 & 39 \\
\hline$\pi$ & 42,3 & 3,1 & 21 & 9,3 & 2,7 & 21 \\
\hline q & 40,3 & 2,5 & 19 & 6,1 & 1,8 & 18 \\
\hline $\begin{array}{c}\delta \text { im Vergleich mit } q \\
\text { (P-Wert) }\end{array}$ & 0,03 & & & $<0,01$ & & \\
\hline
\end{tabular}

Tabelle 4: Vergleich der drei Implantationslokalisationen. Die folgenden Messwerte sind in Millimeter angegeben. Signifikante P-Werte sind fett hervorgehoben. Die maximal angegebene Duraimpression bezieht sich auf Implantationen mit Abstandshaltern (BCI-lifts) *. Ein Patient war auf Grund eines Hämatoms nicht beurteilbar **.

\begin{tabular}{|c|c|c|c|c|c|c|c|c|c|c|c|c|}
\hline & \multicolumn{3}{|c|}{$\begin{array}{l}\text { Dicke der } \\
\text { Schädelkalotte }\end{array}$} & \multicolumn{3}{|c|}{$\begin{array}{l}\text { max. } \\
\text { Impression der } \\
\text { Dura* }\end{array}$} & \multicolumn{3}{|c|}{$\begin{array}{l}\text { Abstand zur } \\
\text { Cochlea }\end{array}$} & \multicolumn{3}{|c|}{$\begin{array}{l}\text { Dicke des } \\
\text { Epikraniums über der } \\
\text { Spule }\end{array}$} \\
\hline & $\varnothing$ & SA & Fälle & $\varnothing$ & SA & Fälle & $\varnothing$ & SA & Fälle & $\varnothing$ & SA & Fälle \\
\hline retrosigmoidal & 6,4 & 2,0 & 52 & 1,4 & 1,0 & 29 & 50,6 & 3,1 & 52 & 8,4 & 3,5 & $51^{* *}$ \\
\hline parietal & 6,1 & 1,7 & 52 & 1,2 & 0,7 & 23 & 58,8 & 4,4 & 52 & 9,7 & 3,6 & $51 * *$ \\
\hline Mastoidresiduum & $N / A$ & & & $\mathrm{~N} / \mathrm{A}$ & & & 41,2 & 3,1 & 35 & 7,7 & 2,9 & 35 \\
\hline$p$ & $>0,05$ & & & $>0,05$ & & & $<0,0$ & & & 0,05 & & \\
\hline
\end{tabular}

\subsection{Publikationen im Rahmen der Dissertation}

Weiss BG, Bertlich M, Scheele R, Canis M, Sohns JM, Ihler F (2017): Radioanatomie potentieller Implantationslokalisationen für ein teilimplantiertes Knochenleitungshörsystem bei Patienten mit vorausgegangener Mastoidektomie. GMS Current Posters in Otorhinolaryngology Head and Neck Surgery, Doc17hno438 [https://www.egms.de/static/pdf/journals/cpo/2017-13/cpo001641.pdf]

Weiss BG, Bertlich M, Scheele R, Canis M, Jakob M, Sohns JM, Ihler F (2017): Systematic radiographic evaluation of three potential implantation sites for a semi-implantable bone conduction device in 52 patients after previous mastoid surgery. Eur Arch Otorhinolaryngol 274, 3001-3009 


\section{$7 \quad$ Literaturverzeichnis}

Albrektsson T, Brånemark P, Hansson H, Lindström J (1981): Osseointegrated titanium implants. Requirements for ensuring a long-lasting, direct bone-to-implant anchorage in man. Acta Orthop Scand $\underline{52}, 70-155$

Amin N, Aymat-Torrente A (2017): Subdural Hematoma: A Rare Adverse Complication From Bone-Anchored Hearing Aid Placement. Otol Neurotol $\underline{38}$, 360-363

Barbara M, Perotti M, Gioia B, Volpini L, Monini S (2013): Transcutaneous bone-conduction hearing device: audiological and surgical aspects in a first series of patients with mixed hearing loss. Acta Otolaryngol 133, 1058-1064

Boenninghaus H-G, Lenarz T: Hals-Nasen-Ohren-Heilkunde: 13. Auflage; Springer, Heidelberg 2007

Bosman A, Snik A, van der Pouw C, Mylanus E, Cremers C (2001): Audiometric evaluation of bilaterally fitted bone-anchored hearing aids. Audiol Neurotol $\underline{40}, 67-158$

Canis M, Ihler F, Blum J, Matthias C (2013): CT-gestützte Navigation zur retrosigmoidalen Implantation der Bonebridge. HNO $\underline{61}, 1038-1044$

Ceit. http://www1.ceit.es/cg/BBFastView/; Zugriff am 11.06.2017

Clemente F, Costa M, Monini S, Barbara M (2011): Monitoring of Fixture Osteointegration after BAHA® Implantation. Audiol Neurotol $\underline{16}, 158-163$

Edmiston RC, Aggarwal R, Green KMJ (2015): Bone conduction implants - a rapidly developing field. J Laryngol Amp Otol 129, 936-940

Eeg-Olofsson M, Stenfelt S, Tjellström A, Granström G (2008): Transmission of bone-conducted sound in the human skull measured by cochlear vibrations. Int J Audiol 47, 761-769

Eeg-Olofsson M, Stenfelt S, Taghavi H, Reinfeldt S, Håkansson B, Tengstrand T, Finizia C (2013): Transmission of bone conducted sound - Correlation between hearing perception and cochlear vibration. Hear Res $\underline{306}, 11-20$

Ent USA: Mastoid Surgery. http://www.entusa.com/mastoid_surgery.htm; Zugriff am 25.04.2017

Fiellau-Nikolajsen M (1983): Epidemiology of Secretory Otitis Media a Descriptive Cohort Study. Ann Otol Rhinol Laryngol 2, 172-177

Güldner C, Heinrichs J, Weiß R, Zimmermann AP, Dassinger B, Bien S, Werner JA, Diogo I (2013): Visualisation of the Bonebridge by means of CT and CBCT. Eur J Med Res $\underline{18}, 30$

Gürkov R, Nagel P: BASICS Hals-Nasen-Ohren-Heilkunde. 3. Auflage; Elsevier, Urban \& Fischer, München 2013

Håkansson B, Eeg-Olofsson M, Reinfeldt S, Stenfelt S, Granström G (2008): Percutaneous Versus Transcutaneous Bone Conduction Implant System: A Feasibility Study on a Cadaver Head. Otol Neurotol 29, 1132-1139 
Håkansson B, Reinfeldt S, Eeg-Olofsson M, Östli P, Taghavi H, Adler J, Gabrielsson J, Stenfelt S, Granström G (2010): A novel bone conduction implant (BCI): Engineering aspects and pre-clinical studies. Int J Audiol 49, 203-215

Hassepass F, Bulla S, Aschendorff A, Maier W, Traser L, Steinmetz C, Wesarg T, Arndt S (2015): The bonebridge as a transcutaneous bone conduction hearing system: preliminary surgical and audiological results in children and adolescents. Eur Arch Otorhinolaryngol 272, 22352241

Hobson JC, Roper AJ, Andrew R, Rothera MP, Hill P, Green KM (2010): Complications of boneanchored hearing aid implantation. J Laryngol Amp Otol 124, 132-136

Holtmann H, Hackenberg B, Wilhelm B: BASICS Mund-, Kiefer- und Plastische Gesichtschirurgie. 1. Auflage; Elsevier, Urban \& Fischer, München 2016

Huber A, Sim J, Xie Y, Chatzimichalis M, Ullrich O, Röösli C (2013): The Bonebridge: preclinical evaluation of a new transcutaneously-activated bone anchored hearing device. Hear Res 301, 93-99

Ito T, Röösli C, Kim C, Sim J, Huber A, Probst R (2011): Bone conduction thresholds and skull vibration measured on the teeth during stimulation at different sites on the human head. Audiol Neurotol 16, 12-22

ITZ Medicom. www.itz-medi.com. http://www.itz-medi.com/allgemein/was-ist-ein-pacs/; Zugriff am 27.04.2017

Lang JJ, Samii A (1991): Retrosigmoidal approach to the posterior cranial fossa. An anatomical study. Acta Neurochir $\underline{111}, 53-147$

Law EKC, Bhatia KSS, Tsang WSS, Tong MCF, Shi L (2016): CT pre-operative planning of a new semi-implantable bone conduction hearing device. Eur Radiol 26, 1686-1695

Mathers C, Smith A, Concha M (2000): Global burden of hearing loss in the year 2000. World Health Organ $\underline{130}$

Matsumoto N, Takumi Y, Cho B, Mori K, Usami S, Yamashita M, Hashizume M, Komune S (2015): Template-guided implantation of the Bonebridge: clinical experience. Eur Arch Otorhinolaryngol 272, 3669-3675

MED-EL: Bonebridge TM Information for Surgeons. http://s3.medel.com/pdf/28178_40_BB\%20Information\%20for\%20Surgeons.pdf; Zugriff am 03.04.2017

Paulsen F, Waschke J: Sobotta Atlas der Anatomie des Menschen Kopf. Bd. 3, Kopf, Hals und Neuroanatomie. 23. Auflage; Elsevier, Urban \& Fischer, München 2010

Perez R, Adelman C, Sohmer H (2011): Bone conduction activation through soft tissues following complete immobilization of the ossicular chain, stapes footplate and round window. Hear Res $\underline{280}, 82-85$

Plontke SK, Radetzki F, Seiwerth I, Herzog M, Brandt S, Delank K, Rahne T (2014): Individual computer-assisted 3d planning for surgical placement of a new bone conduction hearing device. Otol Neurotol Off Publ Am Otol Soc Am Neurotol Soc Eur Acad Otol Neurotol $\underline{35}, 1251-1257$

Rahne T, Schilde S, Seiwerth I, Radetzki F, Stoevesandt D, Plontke SK (2016): Mastoid Dimensions in Children and Young Adults: Consequences for the Geometry of Transcutaneous Bone-Conduction Implants. Otol Neurotol 37, 57-61 
Schmidt. http:/ /www.werner-schmidt-wittenberg.de/ohr.html; Zugriff am 26.05.2017

3D Slicer. https://www.slicer.org/; Zugriff am 11.06.2017

Sohn W (2001): Schwerhörigkeit in Deutschland, Repräsentative Hörscreening-Untersuchung bei 2000 Probanden in 11 Allgemeinpraxen. Z Allg Med 그, 143-7

Stenfelt S (2011): Acoustic and physiologic aspects of bone conduction hearing. Adv Otorhinolaryngol $\underline{71}, 10-21$

Tjellström A, Granström G (1994): Long-term follow-up with the bone-anchored hearing aid: a review of the first 100 patients between 1977 and 1985. Ear Nose Throat J $\underline{73}, 4-112$

Todt I, Lamecker H, Ramm H, Frenzel H, Wollenberg B, Beleites T, Zahnert T, Thomas JP, Dazert S, Ernst A (2014): Entwicklung eines CT-Daten-basierten Vibrant-BonebridgeViewers. HNO $\underline{62}, 439-442$

Ucerler H, Govsa F (2006): Asterion as a surgical landmark for lateral cranial base approaches. J Craniomaxillofac Surg $\underline{34}, 415-420$

Wimmer W, Gerber N, Guignard J, Dubach P, Kompis M, Weber S, Caversaccio M (2015): Topographic bone thickness maps for Bonebridge implantations. Eur Arch Otorhinolaryngol $\underline{272}, 1651-1658$

Zahnert T (2011): The differential diagnosis of hearing loss. Dtsch Ärztebl 108, 44-433

Zarowski AJ, Verstraeten N, Somers T, Riff D, Offeciers EF (2011): Headbands, Testbands and Softbands in Preoperative Testing and Application of Bone-Anchored Devices in Adults and Children. Adv Otorhinolaryngol 71, 124-131

Zernotti ME, Sarasty AB (2015): Active Bone Conduction Prosthesis: BonebridgeTM. Int Arch Otorhinolaryngol 19, 343-348 